TRANSACTIONS OF THE

AMERICAN MATHEMATICAL SOCIETY

Volume 363, Number 7, July 2011, Pages 3373-3438

S 0002-9947(2011)05321-3

Article electronically published on February 7, 2011

\title{
QUANTUM MONODROMY AND NONCONCENTRATION NEAR A CLOSED SEMI-HYPERBOLIC ORBIT
}

\author{
HANS CHRISTIANSON
}

\begin{abstract}
For a large class of semiclassical operators $P(h)-z$ which includes Schrödinger operators on manifolds with boundary, we construct the Quantum Monodromy operator $M(z)$ associated to a periodic orbit $\gamma$ of the classical flow. Using estimates relating $M(z)$ and $P(h)-z$, we prove semiclassical estimates for small complex perturbations of $P(h)-z$ in the case $\gamma$ is semihyperbolic. As our main application, we give logarithmic lower bounds on the mass of eigenfunctions away from semi-hyperbolic orbits of the associated classical flow.

As a second application of the Monodromy Operator construction, we prove if $\gamma$ is an elliptic orbit, then $P(h)$ admits quasimodes which are well-localized near $\gamma$.
\end{abstract}

\section{INTRODUCTION}

1.1. Statement of results. To motivate our general results, we first present a few applications. Suppose $(X, g)$ is a compact Riemannian manifold with or without boundary. Let $-\Delta_{g}$ be the Laplace-Beltrami operator on $X$ and assume $u$ solves the eigenvalue problem

$$
-\Delta_{g} u=\lambda^{2} u,\|u\|_{L^{2}(X)}=1 .
$$

Assume $\gamma$ is a closed semi-hyperbolic geodesic satisfying either $\gamma \cap \partial X=\emptyset$ or the reflection at the boundary is transversal. Then if $U$ is a sufficiently small neighbourhood of $\gamma$, we prove

$$
\int_{X \backslash U}|u|^{2} d x \geq \frac{C}{\log |\lambda|},|\lambda| \rightarrow \infty .
$$

From Chr1, Chr1a, we have an application to subexponential decay of $L^{2}$ energy for the damped wave equation: suppose $a(x)$ is positive outside of $U$ and $u$ satisfies

$$
\left\{\begin{array}{l}
\left(\partial_{t}^{2}-\Delta+2 a(x) \partial_{t}\right) u(x, t)=0, \quad(x, t) \in X \times(0, \infty), \\
u(x, 0)=0, \quad \partial_{t} u(x, 0)=f(x) .
\end{array}\right.
$$

Then for every $\epsilon>0$ there exists $C>0$ such that

$$
\left\|\partial_{t} u\right\|_{L^{2}(X)}^{2}+\|\nabla u\|_{L^{2}(X)}^{2} \leq C e^{-t^{1 / 2} / C}\|f\|_{H^{\epsilon}(X)}^{2} .
$$

An example in $\mathrm{BuCh}$ shows this estimate is in general sharp.

In addition, we have two dispersive type estimates from Chr2. The first is a local smoothing estimate for the Schrödinger equation. Suppose $X$ is a noncompact

Received by the editors February 3, 2009.

2010 Mathematics Subject Classification. Primary 58J42; Secondary 35P20, 35B34.

(C)2011 American Mathematical Society Reverts to public domain 28 years from publication 
manifold which is asymptotically Euclidean and that there is a hyperbolic closed geodesic $\gamma \subset U \Subset X$. Then for every $\epsilon>0$ there exists $C>0$ such that

$$
\int_{0}^{T}\left\|\rho_{s} e^{i t\left(\Delta_{g}-V(x)\right)} u_{0}\right\|_{H^{1 / 2-\epsilon}(X)}^{2} d t \leq C\left\|u_{0}\right\|_{L^{2}(X)}^{2},
$$

where $\rho_{s} \in \mathcal{C}^{\infty}(M)$ satisfies

$$
\rho_{s}(x) \equiv\left\langle d_{g}\left(x, x_{0}\right)\right\rangle^{-s}, s>1 / 2
$$

for $x_{0}$ fixed and $x$ outside a compact set, and $V \in \mathcal{C}^{\infty}(M), 0 \leq V \leq C$ satisfies

$$
|\nabla V| \leq C\left\langle\operatorname{dist}\left(x, x_{0}\right)\right\rangle^{-1-\delta}
$$

for some $\delta>0$.

The second dispersive estimate is a subexponential local energy decay rate for solutions to the wave equation in odd dimensions $n \geq 3$. Suppose $X$ is a noncompact Riemannian manifold which is Euclidean outside a compact set, and suppose $u$ solves

$$
\left\{\begin{array}{l}
\left(-D_{t}^{2}-\Delta_{g}+V(x)\right) u(x, t)=0, X \times[0, \infty), \\
u(x, 0)=u_{0} \in H^{1+s}(X), D_{t} u(x, 0)=u_{1} \in H^{s}(X), s>0
\end{array}\right.
$$

for $u_{0}$ and $u_{1}$ smooth, compactly supported, where $V \in \mathcal{C}^{\infty}(M)$ satisfies

$$
\exp \left(-\operatorname{dist}_{g}\left(x, x_{0}\right)^{2}\right) V=o(1) .
$$

Let $\psi \in \mathcal{C}^{\infty}(X)$ satisfy

$$
\psi \equiv \exp \left(-\operatorname{dist}_{g}\left(x, x_{0}\right)^{2}\right)
$$

for $x$ outside a compact set and $x_{0}$ fixed. Then for every $\epsilon>0$ there exists $C>0$ such that 1

$$
\begin{aligned}
& \left\|\psi \partial_{t} u\right\|_{L^{2}(X)}^{2}+\|\psi u\|_{H^{1}(X)}^{2} \\
& \quad \leq C e^{-t^{1 / 2} / C}\left(\left\|\partial_{t} u(x, 0)\right\|_{H^{\epsilon}(X)}^{2}+\|u(x, 0)\|_{H^{1+\epsilon}(X)}^{2}\right) .
\end{aligned}
$$

For the general statement of results, let $X$ be a smooth, compact manifold. In this introduction, we state the Main Theorem only in the case $\partial X=\emptyset$. The case with boundary will be considered in $₫ 3$. We take $P(h) \in \Psi_{h}^{k, 0}$ for $k \geq 1$ (see 92 for definitions) and assume $P(h)$ is of real principal type. That is, if $p=\mathcal{C}^{\infty}\left(T^{*} X\right)$ is the principal symbol of $P(h)$, then $p$ is real-valued, independent of $h$. Assume $p^{-1}(E)$ is a smooth, compact hypersurface and $d p(x, \xi) \neq 0$ for energies $E$ near 0 . We assume $p$ is elliptic outside of a compact subset of $T^{*} X$ : there exists $C>0$ such that

$$
|\xi|>C \Longrightarrow p(x, \xi) \geq\langle\xi\rangle^{k} / C .
$$

Let $\Phi_{t}=\exp t H_{p}$ be the Hamiltonian flow of $p$, and suppose $\Phi_{t}$ has a closed, semi-hyperbolic orbit $\gamma \subset\{p=0\}$ of period $T$. The assumption that $\gamma$ be semihyperbolic means if $N$ is a Poincaré section for $\gamma$ and $S: N \rightarrow S(N)$ is the Poincaré map, then the linearization of $S, d S(0)$, is nondegenerate and has at least

\footnotetext{
${ }^{1}$ Using the recent work of Nonnenmacher-Zworski NoZw1, NoZw2, this has now been improved to exponential decay $\mathrm{Chr}$.
} 
one eigenvalue off the unit circle. For the eigenvalues of modulus 1 we also require the following nonresonance assumption for the Poincaré map at the energy 0:

$$
\left\{\begin{array}{l}
\text { if } e^{ \pm i \alpha_{1}}, e^{ \pm i \alpha_{2}}, \ldots, e^{ \pm i \alpha_{k}} \text { are eigenvalues of modulus } 1, \text { then } \\
\alpha_{1}, \alpha_{2}, \ldots, \alpha_{k} \text { are independent over } \pi \mathbb{Z} \text {. }
\end{array}\right.
$$

Our Main Theorem is that for a family of eigenfunctions $u(h)$ for $P(h)$,

$$
P(h) u(h)=E(h) u(h), \quad E(h) \rightarrow 0 \text { as } h \rightarrow 0,
$$

and $u(h)$ has its mass concentrated away from $\gamma$. This is made precise in the following theorem.

Main Theorem. Let $A \in \Psi_{h}^{0,0}(X)$ be a pseudodifferential operator whose principal symbol is 1 near $\gamma$ and 0 away from $\gamma$. There exist constants $h_{0}>0$ and $C>0$ such that

$$
\|u\| \leq C \frac{\log (1 / h)}{h}\|P(h) u\|+C \sqrt{\log (1 / h)}\|(I-A) u\|
$$

uniformly in $0<h<h_{0}$, where the norms are $L^{2}$-norms on $X$. In particular, if $u(h)$ satisfies

$$
\left\{\begin{array}{l}
P(h) u(h)=\mathcal{O}\left(h^{\infty}\right) \\
\|u(h)\|_{L^{2}(X)}=1
\end{array}\right.
$$

then

$$
\|(I-A) u\|_{L^{2}(X)} \geq \frac{1}{C}(\log (1 / h))^{-1 / 2}, \quad 0<h<h_{0} .
$$

Remark 1.1. In $₫ 3$ we assume $P(h) \in \operatorname{Diff}_{h}^{2}(X)$ is a differential operator on $X$ and that $\partial X$ is noncharacteristic with respect to the principal symbol of $P(h)$. Then a similar conclusion to the Main Theorem holds (see Main Theorem' in that section).

Remark 1.2. In $\$ 10$, we give a partial converse to the Main Theorem in Theorem 7. That is, the techniques of the proof of the Main Theorem are used to show that if the periodic orbit $\gamma$ is elliptic, then $P(h)$ admits quasimodes which are welllocalized to $\gamma$. This result is well known in the literature (see, for example, Ral, $\mathrm{CdV}$, and $\mathrm{CaPo}$ and the references cited therein); however, to our knowledge the use of the Quantum Monodromy operator to construct these quasimodes is new.

Remark 1.3. We interpret the assumption that $\gamma$ be semi-hyperbolic as follows: if $\gamma$ were fully hyperbolic and $\partial X=\emptyset$, then we know from [Chr1] that the mass of eigenfunctions cannot concentrate very rapidly (as the eigenvalue goes to infinity) near $\gamma$. However, we know from Theorem 7 below that if $\gamma$ were fully elliptic, then there are at least approximate eigenfunctions with all their mass concentrated near $\gamma$. The rough heuristic is that if $\gamma$ is hyperbolic in at least one direction in phase space and a sequence of eigenfunctions were to concentrate rapidly near $\gamma$, then they would have to do so in a lower-dimensional set, a clear violation of the uncertainty principle 2 Of course the proof is quite a bit more technical. The nonresonance assumption on the elliptic eigenvalues is necessary in order to assume there are no other periodic orbits in a neighbourhood of $\gamma$ in the set $\{p=0\}$. This in turn implies (see, for instance, AbMa, Theorem 28.5]) that there is a range of energy surfaces $\{p=z\}$ near $z=0$ in which there is an isolated periodic semi-hyperbolic orbit. The family of these orbits is diffeomorphic to a cylinder.

\footnotetext{
${ }^{2} \mathrm{See}[\mathrm{ChWu}$ for recent related work for some examples of degenerate hyperbolic orbits.
} 
Remark 1.4. The estimates in this work are all microlocal in nature; hence we lose nothing by assuming $X$ is compact. In order to apply these estimates in the case of noncompact manifolds, we assume $P$ is elliptic outside a compact set (that is, $|p| \geq\langle\xi\rangle^{k} / C$ for $\left.|(x, \xi)| \geq C\right)$ and the geometry is nontrapping outside of a compact submanifold and then apply our results there. See Chr2 for more on this.

The Main Theorem is similar to [Chr1, Main Theorem] with three generalizations, namely that we no longer assume the linearized Poincaré map has no negative eigenvalues, we allow some eigenvalues of modulus 1 , and in $\$ 3$ we allow $\gamma$ to reflect transversally off $\partial X$ with some extra assumptions on $P(h)$. This allows the study of, for example, billiard problems in any dimension. The problems encountered in Chr1 with these cases come from attempting to put $p$ into a normal form in a neighbourhood of $\gamma \subset T^{*} X$.

The motivation for the proof in this paper is to reduce the problem of studying the resolvent $(P-z)^{-1}$ in a microlocal neighbourhood of $\gamma$ to studying a related operator on the Poincaré section $N$.

If we identify $N$ with $T_{0}^{*} N \simeq \mathbb{R}^{2 n-2}$ near 0 , we are led to study operators acting on $L^{2}(V)$, where $V \subset \mathbb{R}^{n-1}$. In the course of this work, we will see that the relevant object of study is the Quantum Monodromy operator $M(z): L^{2}(V) \rightarrow L^{2}(V)$. By setting up a Grushin problem in a neighbourhood of

$$
\gamma \times(0,0) \subset T^{*} X \times T^{*} \mathbb{R}^{n-1},
$$

and using the microlocal inverse constructed by Sjöstrand-Zworski in SjZw1, we will see it is sufficient to bound $\|I-M(z)\|_{L^{2}(V) \rightarrow L^{2}(V)}$ from below. This will result in the following theorem, which is the main technical result of this paper.

Theorem 1. Suppose $P \in \Psi_{h}^{k, 0}$ is a semiclassical pseudodifferential operator of real principal type satisfying all of the assumptions of the introduction, and assume $\gamma \cap \partial X=\emptyset$. Then there exist positive constants $C, c_{0}, h_{0}, \epsilon_{0}$, and a positive integer $N$ such that for $0<h<h_{0}, z \in\left[-\epsilon_{0}, \epsilon_{0}\right]+i\left(-c_{0} h, c_{0} h\right)$, and if $u \in L^{2}(X)$ has $h$-wavefront set sufficiently close to $\gamma$, then

$$
\|(P-z) u\|_{L^{2}(X)} \geq C^{-1} h^{N}\|u\|_{L^{2}(X)} .
$$

Theorem 1 allows us to add a complex absorption term of order $h$ supported away from $\gamma$. Let $a \in \mathcal{C}^{\infty}\left(T^{*} X\right)$ equal 0 in a neighbourhood of $\gamma$ and 1 away from $\gamma$, and define

$$
Q(z) u=P(h)-z-i h C a^{w}
$$

for a constant $C>0$ to be chosen later. Then a semiclassical adaptation of the "three-lines" theorem from complex analysis will allow us to deduce the following estimate.

Theorem 2. Suppose $Q(z)$ is given by (1.4), and let

$$
z \in\left[-\epsilon_{0} / 2, \epsilon_{0} / 2\right]+i(-c h / \log (1 / h), c h / \log (1 / h))
$$

for $\epsilon_{0}, c>0$ sufficiently small. Then there is $h_{0}>0$ and $0<C<\infty$ such that for $0<h<h_{0}$,

$$
\left\|Q(z)^{-1}\right\|_{L^{2}(X) \rightarrow L^{2}(X)} \leq C \frac{\log (1 / h)}{h} .
$$


As an intermediate step to proving Theorem 2, we first prove a similar statement about an operator with a larger absorbing term. Let $W \in \mathcal{C}^{\infty}\left(T^{*} X\right), W \equiv 0$ in a small neighbourhood of $\gamma$ and $W \equiv 1$ away from $\gamma$. We define a perturbed family of operators

$$
\widetilde{Q}(z)=P(h)-z-i W^{w} .
$$

We have the following theorem.

Theorem 3. Suppose $\widetilde{Q}(z)$ is as above, $z \in\left[-\epsilon_{0} / 2, \epsilon_{0} / 2\right]$, and $W \equiv 1$ outside a sufficiently small neighbourhood of $\gamma$. Then there is $h_{0}>0$ and $0<C<\infty$ such that for $0<h<h_{0}$,

$$
\left\|\widetilde{Q}(z)^{-1}\right\|_{L^{2}(X) \rightarrow L^{2}(X)} \leq C \frac{\log (1 / h)}{h} .
$$

If $\varphi \in \mathcal{C}_{c}^{\infty}(X)$ is supported away from $\gamma$, then

$$
\left\|\widetilde{Q}(z)^{-1} \varphi\right\|_{L^{2}(X) \rightarrow L^{2}(X)} \leq C \frac{\sqrt{\log (1 / h)}}{h} .
$$

Theorem 2 follows from Theorem 3 using the control theory arguments exactly as in Chr1a].

1.2. Examples. There are many examples in which the hypotheses of the theorem are satisfied, the simplest of which is the case in which $p=|\xi|^{2}-E(h)$ for $E(h)>0$. Then the Hamiltonian flow of $p$ is the geodesic flow, so if the geodesic flow has a closed semi-hyperbolic orbit, there is nonconcentration of eigenfunctions, $u(h)$, for the equation

$$
-h^{2} \Delta u(h)=E(h) u(h) .
$$

Another example of such a $p$ is the case $p=|\xi|^{2}+V(x)$, where $V(x)$ is a confining potential with two "bumps" or "obstacles" in the lowest energy level (see Figure 11. In the appendix to [Sjö it is shown that for an interval of energies $V(x) \sim$ 0 , there is a closed hyperbolic orbit $\gamma$ of the Hamiltonian flow which "reflects" off the bumps (see Figure 21). Complex hyperbolic orbits may be constructed by considering 3-dimensional hyperbolic billiard problems (see, for example, AuMa, $\S 2]$ ). In addition, Proposition 4.3 from Chr1 gives a somewhat artificial means of constructing a manifold diffeomorphic to a neighbourhood in $T^{*} \mathbb{S}_{(t, \tau)}^{1} \times T^{*} \mathbb{R}_{(x, \xi)}^{n-1}$ which contains a hyperbolic orbit $\gamma$ by starting with the Poincaré map that $\gamma$ is required to have.

In the appendix, we examine the Riemannian manifold

$$
M=\mathbb{R}_{x} / \mathbb{Z} \times \mathbb{R}_{y} \times \mathbb{R}_{z}
$$

equipped with the metric

$$
d s^{2}=\cosh ^{2} y\left(2 z^{4}-z^{2}+1\right)^{2} d x^{2}+d y^{2}+d z^{2},
$$

which has a semi-hyperbolic closed geodesic at $y=z=0$ and two hyperbolic closed geodesics at $y=0, z= \pm 1 / 2$. Restricting $y$ and $z$ to compact intervals yields a compact manifold satisfying the hypotheses of the Main Theorem, while the noncompact manifold provides a model for possibly extending the dispersivetype estimates to the semi-hyperbolic case. 


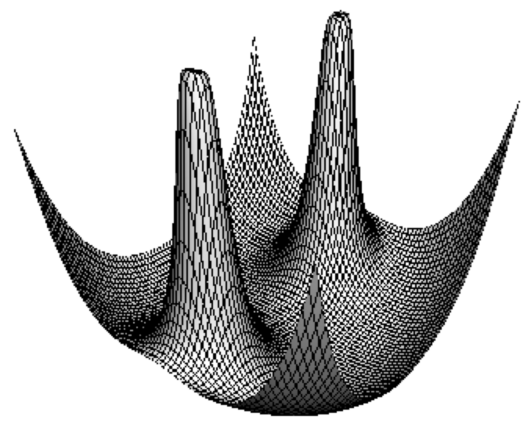

Figure 1. A confining potential $V(x)$ with two bumps at the lowest energy level $E<0$.

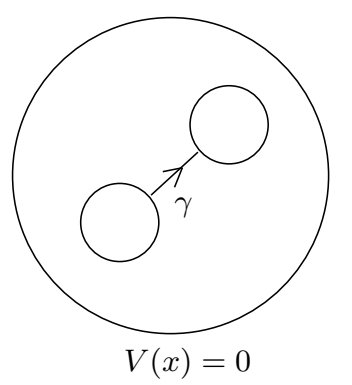

Figure 2. The level set $V(x)=0$ and the closed hyperbolic orbit $\gamma$ reflecting off the "soft" boundary.

1.3. Organization. This work is organized as follows. In $\ 2$, we recall basic definitions and properties of $h$-pseudodifferential operators on manifolds. In $\$ 3$ we first state the versions of Theorems 1, 2, and the Main Theorem in the case of a compact manifold with boundary, then review the classical picture of a closed orbit reflecting transversally off the boundary, and prove a propagation of singularities result at the boundary. 连 gives the definition and basic facts about the Quantum Monodromy operator $M(z)$, while $\$ 5$ shows how $M(z)$ arises naturally in the context of a Grushin problem. 6 presents the main ideas of the proof of Theorem[1 by considering a model. In $\$ \$ 7,8$ the proof of Theorem 1 is presented, while the proof of Theorem 2 and the Main Theorem is reserved for \$9] Finally, in 910 we show how the Monodromy Operator construction can be used to construct well-localized quasimodes if $\gamma$ is elliptic. In the appendix, we provide a concrete example of a semi-hyperbolic orbit.

\section{Preliminaries}

The material in this section is standard (see, for example, DiSj, EvZw for the semiclassical calculus, or Hor1, Hor3 for the classical case), but we include it here for the reader's convenience.

2.1. h-Pseudodifferential operators on manifolds. We will be operating on half-densities,

$$
u(x)|d x|^{\frac{1}{2}} \in \mathcal{C}^{\infty}\left(X, \Omega_{X}^{\frac{1}{2}}\right)
$$

with the informal change of variables formula

$$
u(x)|d x|^{\frac{1}{2}}=v(y)|d y|^{\frac{1}{2}}, \text { for } y=\kappa(x) \Leftrightarrow v(\kappa(x))\left|\kappa^{\prime}(x)\right|^{\frac{1}{2}}=u(x) .
$$

By symbols on $X$ we mean

$$
\begin{aligned}
& \mathcal{S}^{k, m}\left(T^{*} X, \Omega_{T^{*} X}^{\frac{1}{2}}\right) \\
& \quad:=\left\{a \in \mathcal{C}^{\infty}\left(T^{*} X \times(0,1], \Omega_{T^{*} X}^{\frac{1}{2}}\right):\left|\partial_{x}^{\alpha} \partial_{\xi}^{\beta} a(x, \xi ; h)\right| \leq C_{\alpha \beta} h^{-m}\langle\xi\rangle^{k-|\beta|}\right\} .
\end{aligned}
$$

We remark that the symbols we consider are half-densities on the cotangent bundle with the natural symplectic structure, and in the course of this work we will 
use only symplectic changes of variables on $T^{*} X$. Consequently the change of variables formula is invariant on symbols: if $\kappa: T^{*} X \rightarrow T^{*} X$ is symplectic and $a \in \mathcal{S}^{k, m}\left(T^{*} X, \Omega_{T^{*} X}^{\frac{1}{2}}\right)$, then

$$
\begin{aligned}
\kappa^{*}\left[a(x, \xi)|d \xi \wedge d x|^{\frac{1}{2}}\right] & =a(\kappa(x, \xi))\left|\kappa^{*} d \xi \wedge d x\right|^{\frac{1}{2}} \\
& =a(\kappa(x, \xi))|d \xi \wedge d x|^{\frac{1}{2}}
\end{aligned}
$$

Hence we don't keep track of the $|d \xi \wedge d x|^{\frac{1}{2}}$ except where confusion may arise.

There is a corresponding class of pseudodifferential operators $\Psi_{h}^{k, m}\left(X, \Omega_{X}^{\frac{1}{2}}\right)$ acting on half-densities defined by the local formula (Weyl calculus) in $\mathbb{R}^{n}$ :

$$
\mathrm{Op}_{h}^{w}(a) u(x)=\frac{1}{(2 \pi h)^{n}} \iint a\left(\frac{x+y}{2}, \xi ; h\right) e^{i\langle x-y, \xi\rangle / h} u(y) d y d \xi .
$$

We will occasionally use the shorthand notation $a^{w}:=\mathrm{Op}_{h}^{w}(a)$ and $A:=\mathrm{Op}_{h}^{w}(a)$ when there is no ambiguity in doing so. We also use the notation $P \in \operatorname{Diff}_{h}^{k}$ when $P$ is a semiclassical differential operator.

We have the principal symbol map

$$
\sigma_{h}: \Psi_{h}^{k, m}\left(X, \Omega_{X}^{\frac{1}{2}}\right) \rightarrow \mathcal{S}^{k, m} / \mathcal{S}^{k, m-1}\left(T^{*} X, \Omega_{T^{*} X}^{\frac{1}{2}}\right),
$$

which gives the left inverse of $\mathrm{Op}_{h}^{w}$ in the sense that

$$
\sigma_{h} \circ \mathrm{Op}_{h}^{w}: \mathcal{S}^{k, m} \rightarrow \mathcal{S}^{k, m} / \mathcal{S}^{k, m-1}
$$

is the natural projection. Acting on half-densities in the Weyl calculus, the principal symbol is actually well-defined in $\mathcal{S}^{k, m} / \mathcal{S}^{k, m-2}$, that is, up to $\mathcal{O}\left(h^{2}\right)$ in $h$ (see, [SjZw1, Appendix]).

We will use the notion of wave front sets for pseudodifferential operators on manifolds. If $a \in \mathcal{S}^{k, m}\left(T^{*} X, \Omega_{T^{*} X}^{\frac{1}{2}}\right)$, we define the singular support or essential support for $a$ :

$$
\operatorname{ess-supp}_{h} a \subset T^{*} X \sqcup \mathbb{S}^{*} X,
$$

where $\mathbb{S}^{*} X=\left(T^{*} X \backslash\{0\}\right) / \mathbb{R}_{+}$is the cosphere bundle (quotient taken with respect to the usual multiplication in the fibers), and the union is disjoint. ess-supp ${ }_{h} a$ is defined using complements:

$$
\begin{aligned}
& :=\quad \complement\left\{(x, \xi) \in T^{*} X: \exists \epsilon>0, \partial_{x}^{\alpha} \partial_{\xi}^{\beta} a\left(x^{\prime}, \xi^{\prime}\right)=\mathcal{O}\left(h^{\infty}\right), d\left(x, x^{\prime}\right)+\left|\xi-\xi^{\prime}\right|<\epsilon\right\} \\
& \quad \cup \complement\left\{(x, \xi) \in T^{*} X \backslash 0: \exists \epsilon>0, \partial_{x}^{\alpha} \partial_{\xi}^{\beta} a\left(x^{\prime}, \xi^{\prime}\right)=\mathcal{O}\left(h^{\infty}\langle\xi\rangle^{-\infty}\right),\right. \\
& \left.\quad d\left(x, x^{\prime}\right)+1 /\left|\xi^{\prime}\right|+|\xi /| \xi\left|-\xi^{\prime} /\right| \xi^{\prime}||<\epsilon\right\} / \mathbb{R}_{+} .
\end{aligned}
$$

We then define the wave front set of a pseudodifferential operator $A \in \Psi_{h}^{k, m}\left(X, \Omega_{X}^{\frac{1}{2}}\right)$ :

$$
\mathrm{WF}_{h}(A):=\operatorname{ess} \operatorname{supp}_{h}(a) \text {, for } A=\mathrm{Op}_{h}^{w}(a) \text {. }
$$

Finally for distributional half-densities $u \in \mathcal{C}^{\infty}\left((0,1]_{h}, \mathcal{D}^{\prime}\left(X, \Omega_{X}^{\frac{1}{2}}\right)\right)$ such that there is an $N_{0}$ so that $h^{N_{0}} u$ is bounded in $\mathcal{D}^{\prime}\left(X, \Omega_{X}^{\frac{1}{2}}\right)$, we can define the semiclassical 
wave front set of $u$, again by a complement:

$$
\begin{aligned}
\mathrm{WF}_{h}(u):=\quad & \quad\left\{(x, \xi): \exists A \in \Psi_{h}^{0,0}, \text { with } \sigma_{h}(A)(x, \xi) \neq 0,\right. \\
& \text { and } \left.A u \in h^{\infty} \mathcal{C}^{\infty}\left((0,1]_{h}, \mathcal{C}^{\infty}\left(X, \Omega_{X}^{\frac{1}{2}}\right)\right)\right\} .
\end{aligned}
$$

For $A=\mathrm{Op}_{h}^{w}(a)$ and $B=\mathrm{Op}_{h}^{w}(b), a \in \mathcal{S}^{k, m}, b \in \mathcal{S}^{k^{\prime}, m^{\prime}}$, we have the composition formula

$$
A \circ B=\mathrm{Op}_{h}^{w}(a \# b),
$$

where

$$
\mathcal{S}^{k+k^{\prime}, m+m^{\prime}} \ni a \# b(x, \xi):=\left.e^{\frac{i h}{2} \omega\left(D_{x}, D_{\xi} ; D_{y}, D_{\eta}\right)}(a(x, \xi) b(y, \eta))\right|_{\substack{x=y \\ \xi=\eta}},
$$

with $\omega$ the standard symplectic form.

We will need the definition of microlocal equivalence of operators. Suppose $T: \mathcal{C}^{\infty}\left(X, \Omega_{X}^{\frac{1}{2}}\right) \rightarrow \mathcal{C}^{\infty}\left(X, \Omega_{X}^{\frac{1}{2}}\right)$ and that for any seminorm $\|\cdot\|_{1}$ on $\mathcal{C}^{\infty}\left(X, \Omega_{X}^{\frac{1}{2}}\right)$ there is a second seminorm $\|\cdot\|_{2}$ on $\mathcal{C}^{\infty}\left(X, \Omega_{X}^{\frac{1}{2}}\right)$ such that

$$
\|T u\|_{1}=\mathcal{O}\left(h^{-M_{0}}\right)\|u\|_{2}
$$

for some $M_{0}$ fixed. Then we say $T$ is semiclassically tempered. We assume for the rest of this work that all operators satisfy this condition. Let $U, V \subset T^{*} X$ be open precompact sets. We think of operators defined microlocally near $V \times U$ as equivalence classes of tempered operators. The equivalence relation is

$$
T \sim T^{\prime} \Longleftrightarrow A\left(T-T^{\prime}\right) B=\mathcal{O}\left(h^{\infty}\right): \mathcal{D}^{\prime}\left(X, \Omega_{X}^{\frac{1}{2}}\right) \rightarrow \mathcal{C}^{\infty}\left(X, \Omega_{X}^{\frac{1}{2}}\right)
$$

for any $A, B \in \Psi_{h}^{0,0}\left(X, \Omega_{X}^{\frac{1}{2}}\right)$ such that

$$
\begin{aligned}
& \mathrm{WF}_{h}(A) \subset \widetilde{V}, \quad \operatorname{WF}_{h}(B) \subset \widetilde{U}, \text { with } \widetilde{V}, \widetilde{U} \text { open and } \\
& \bar{V} \Subset \widetilde{V} \Subset T^{*} X, \quad \bar{U} \Subset \widetilde{U} \Subset T^{*} X .
\end{aligned}
$$

In the course of this work, when we say $P=Q$ microlocally near $V \times U$, we mean for any $A, B$ as above,

$$
A P B-A Q B=\mathcal{O}_{L^{2} \rightarrow L^{2}}\left(h^{\infty}\right),
$$

or in any other norm by the assumed precompactness of $U$ and $V$. Similarly, we say $B=T^{-1}$ on $V \times U$ if $B T=I$ microlocally near $V \times V$ and $T B=I$ microlocally near $U \times U$.

2.2. Symbols with 2 parameters. We will use the following results on symbols with two parameters (for proofs, see SjZw3 and Chr1]). We will only use symbol spaces with two parameters in the context of microlocal estimates, in which case we may assume we are working in an open subset of $\mathbb{R}^{2 n}$. We define the following spaces of symbols with two parameters:

$$
\begin{aligned}
\mathcal{S}^{k, m, \widetilde{m}}\left(\mathbb{R}^{2 n}\right):=\{a & \in \mathcal{C}^{\infty}\left(\mathbb{R}^{2 n} \times(0,1]^{2}\right): \\
& \left.\left|\partial_{x}^{\alpha} \partial_{\xi}^{\beta} a(x, \xi ; h, \tilde{h})\right| \leq C_{\alpha \beta} h^{-m} \tilde{h}^{-\widetilde{m}}\langle\xi\rangle^{k-|\beta|}\right\} .
\end{aligned}
$$


For the applications in this work, we assume $\tilde{h}>h$ and define the scaled spaces:

$$
\begin{aligned}
\mathcal{S}_{\delta}^{k, m, \widetilde{m}}\left(\mathbb{R}^{2 n}\right):= & \left\{a \in \mathcal{C}^{\infty}\left(\mathbb{R}^{2 n} \times(0,1]^{2}\right):\right. \\
& \left.\left|\partial_{x}^{\alpha} \partial_{\xi}^{\beta} a(x, \xi ; h, \tilde{h})\right| \leq C_{\alpha \beta} h^{-m} \tilde{h}^{-\widetilde{m}}\left(\frac{\tilde{h}}{h}\right)^{\delta(|\alpha|+|\beta|)}\langle\xi\rangle^{k-|\beta|}\right\} .
\end{aligned}
$$

As before, we have the corresponding spaces of semiclassical pseudodifferential operators $\Psi^{k, m, \widetilde{m}}$ and $\Psi_{\delta}^{k, m, \widetilde{m}}$, where we will usually add a subscript of $h$ or $\tilde{h}$ to indicate which parameter is used in the quantization. The relationship between $\Psi_{h}$ and $\Psi_{\tilde{h}}$ is given in the following obvious lemma.

Lemma 2.1. Let $a \in \mathcal{S}_{0}^{k, m, \tilde{m}}$ and set

$$
b(X, \Xi)=a\left((h / \tilde{h})^{\frac{1}{2}} X,(h / \tilde{h})^{\frac{1}{2}} \Xi\right) \in \mathcal{S}_{-\frac{1}{2}}^{k, m, \tilde{m}} .
$$

The linear operator $T_{h, \tilde{h}} u(X)=(h / \tilde{h})^{\frac{n}{4}} u\left((h / \tilde{h})^{\frac{1}{2}} X\right)$ is unitary on $L^{2}$, and

$$
\mathrm{Op}_{\tilde{h}}^{w}(b) T_{h, \tilde{h}} u=T_{h, \tilde{h}} \mathrm{Op}_{h}^{w}(a) u .
$$

We have the following microlocal commutator lemma.

Lemma 2.2. Suppose $a \in \mathcal{S}_{0}^{-\infty, 0,0}, b \in \mathcal{S}_{-\frac{1}{2}}^{-\infty, m, \widetilde{m}}$, and $\tilde{h}>h$.

(a) If $A=\mathrm{Op}_{\tilde{h}}^{w}(a)$ and $B=\mathrm{Op}_{\tilde{h}}^{w}(b)$, then

$$
[A, B]=h^{-m} \tilde{h}^{-\tilde{m}}\left(\frac{\tilde{h}}{i} \mathrm{Op}_{\tilde{h}}^{w}(\{a, b\})+\mathcal{O}\left(h^{3 / 2} \tilde{h}^{3 / 2}\right)\right) .
$$

(b) More generally, for each $l>1$,

$$
\operatorname{ad}_{A}^{l} B=h^{-m} \tilde{h}^{-\tilde{m}} \mathcal{O}_{L^{2} \rightarrow L^{2}}\left(h \tilde{h}^{l-1}\right) .
$$

(c) If $a \in \mathcal{S}_{0}^{-\infty, 0,0}, b \in \mathcal{S}_{\frac{1}{2}}^{-\infty, m, \tilde{m}}, \tilde{h}>h, A=\mathrm{Op}_{h}^{w}(a)$, and $B=\mathrm{Op}_{h}^{w}(b)$, then

$$
[A, B]=h^{-m} \tilde{h}^{-\tilde{m}}\left(\frac{h}{i} \mathrm{Op}_{h}^{w}(\{a, b\})+\mathcal{O}\left(h^{3 / 2} \tilde{h}^{3 / 2}\right)\right) .
$$

\section{ManifoldS With BOUNDARY AND PROPAGATION OF SINGULARITIES}

In this section, $X$ is a smooth, compact, $n$-dimensional manifold with boundary. We assume $P \in \operatorname{Diff}_{h}^{2,0}$ is a second-order differential operator whose principal symbol $p$ is a quadratic form in $\xi$ :

$$
p=\sum_{i, j} a^{i j}(x) \xi_{i} \xi_{j}-1,
$$

and we assume $\partial X$ is noncharacteristic with respect to $p$. We adopt a microlocal viewpoint in which $\partial X$ is identified locally with a noncharacteristic hypersurface $Y \subset \mathbb{R}^{n}$. Our local model for $X$ near $Y$ is $X=\mathbb{R}^{n}$ with $Y=\left\{x \in \mathbb{R}^{n}: x_{1}=0\right\}$. If $\gamma$ is a (broken) periodic bicharacteristic in $\{p=0\}$, we implicitly identify $\gamma$ 
with its projection $\pi(\gamma)$ onto $X$, which we think of as a unit speed geodesic. Here $\pi: T^{*} X \rightarrow X$ is the usual projection. We study the boundary value problem

$$
\left\{\begin{array}{l}
(P-z) u=f \text { in } X \\
u=0 \text { on } Y,
\end{array}\right.
$$

in a neighbourhood of a closed bicharacteristic for the flow of $H_{p}$ reflecting transversally off $Y$, and for energies $z$ near 0 . Our final goal is to describe the propagation of singularities at the boundary. First we will prove factorization lemmas and energy estimates near $Y$, and then prove the main result of this section, which is that the microlocal propagator of $P-z$ can be extended in a meaningful way through the reflections at the boundary. The Main Theorem has the following analogue in the case that the bicharacteristic $\gamma$ reflects transversally off $\partial X$ in phase space (see $\S 3.13 .2$ for definitions).

Main Theorem'. Suppose $P(h) \in \operatorname{Diff}_{h}^{2}(X)$ and $\partial X$ is noncharacteristic with respect to the principal symbol of $P(h)$. Assume $\gamma$ makes only transversal reflections with $\partial X$. Let $A \in \Psi_{h, d b}^{0,0}(X)$ be a pseudodifferential operator whose principal symbol is 1 near $\gamma$ and 0 away from $\gamma$. There exist constants $h_{0}>0$ and $C>0$ such that

$$
\|u\| \leq C \frac{\log (1 / h)}{h}\|P(h) u\|+C \sqrt{\log (1 / h)}\|(I-A) u\|
$$

uniformly in $0<h<h_{0}$, where the norms are $L^{2}$-norms on $X$. In particular, if $u(h)$ satisfies

$$
\left\{\begin{array}{l}
P(h) u(h)=\mathcal{O}\left(h^{\infty}\right) \\
\|u(h)\|_{L^{2}(X)}=1
\end{array}\right.
$$

then

$$
\|(I-A) u\|_{L^{2}(X)} \geq \frac{1}{C} \log ((1 / h))^{-\frac{1}{2}}, \quad 0<h<h_{0} .
$$

As in $₫ 1$ Main Theorem' is a consequence of the following versions of Theorems 1 and 2 in the case of a manifold with boundary.

Theorem 1'. Suppose $P(h) \in \operatorname{Diff}_{h}^{2}(X)$ and $\partial X$ is noncharacteristic with respect to the principal symbol of $P(h)$. Assume $\gamma$ makes only transversal reflections with $\partial X$. Then there exist positive constants $C, c_{0}, h_{0}, \epsilon_{0}$, and a positive integer $N$ such that for $0<h<h_{0}, z \in\left[-\epsilon_{0}, \epsilon_{0}\right]+i\left(-c_{0} h, c_{0} h\right)$, if $u \in L^{2}(X)$ has $h$-wavefront set sufficiently close to $\gamma$, then

$$
\|(P-z) u\|_{L^{2}(X)} \geq C^{-1} h^{N}\|u\|_{L^{2}(X)} .
$$

As in the introduction, we add a complex absorbing term: let $a^{w} \in \Psi_{h, d b}$ equal 0 in a neighbourhood of $\gamma$ and 1 away from $\gamma$ (according to the equivalence relation defined in $\$ 3.10$, and define as usual

$$
Q(z) u=P(h)-z-i h C a^{w}
$$

for a constant $C>0$ to be chosen later. We have the following version of Theorem 2.

Theorem $\mathbf{2}^{\prime}$. Suppose $Q(z)$ is given as above, and suppose

$$
z \in\left[-\epsilon_{0} / 2, \epsilon_{0} / 2\right]+i(-c h / \log (1 / h), c h / \log (1 / h))
$$


for $\epsilon_{0}, c>0$ is sufficiently small. Then there is $h_{0}>0$ and $0<C<\infty$ such that for $0<h<h_{0}$,

$$
\left\|Q(z)^{-1}\right\|_{L^{2}(X) \rightarrow L^{2}(X)} \leq C \frac{\log (1 / h)}{h} .
$$

Just as in the introduction, before proving Theorem 2 we first need an auxiliary theorem. Let $W \in \mathcal{C}^{\infty}\left(T^{*} X\right), W \equiv 0$ in a small neighbourhood of $\gamma$ and $W \equiv 1$ away from $\gamma$ (again using the equivalence relations in 3.1). We define a perturbed family of operators

$$
\widetilde{Q}(z)=P(h)-z-i W^{w} .
$$

We have the following theorem.

Theorem 3'. Suppose $\widetilde{Q}(z)$ is as above, $z \in\left[-\epsilon_{0} / 2, \epsilon_{0} / 2\right]$, and $W \equiv 1$ outside a sufficiently small neighbourhood of $\gamma$. Then there is $h_{0}>0$ and $0<C<\infty$ such that for $0<h<h_{0}$,

$$
\left\|\widetilde{Q}(z)^{-1}\right\|_{L^{2}(X) \rightarrow L^{2}(X)} \leq C \frac{\log (1 / h)}{h} .
$$

If $\varphi \in \mathcal{C}_{c}^{\infty}(X)$ is supported away from $\gamma$, then

$$
\left\|\widetilde{Q}(z)^{-1} \varphi\right\|_{L^{2}(X) \rightarrow L^{2}(X)} \leq C \frac{\sqrt{\log (1 / h)}}{h} .
$$

Theorem 2 follows from Theorem 3 using the control theory arguments exactly as in Chr1a.

3.1. Normally differential operators. In this subsection we review some of the technical tools necessary to understand the propagation of singularities through boundary reflections. The calculus described here appears to have originated in the works of Melrose-Sjöstrand MeSj1, MeSj2. The purpose is to mimic as much as possible the properties of the semiclassical calculus in the case with no boundary (composition, commutators, propagation of wavefront sets, etc.); however, it should be noted that the calculus does depend implicitly on the choice of coordinates.

If $X$ is a smooth manifold with boundary, we define pseudodifferential operators which are differential in the normal direction at the boundary microlocally. For a microlocal definition, it suffices to assume $X=\left\{x_{1} \geq 0\right\}$ and $\partial X=\left\{x_{1}=0\right\}$. Then the algebra of pseudodifferential operators which are normally differential at the boundary is defined by the following:

$$
\begin{aligned}
\Psi_{h, d b}^{k, m}\left(X, \Omega_{X}^{\frac{1}{2}}\right)=\left\{A\left(x, h D_{x}\right) \in \Psi_{h}^{k, m}:\right. & \\
& \left.A\left(x, h D_{x}\right)=\sum_{j=0}^{k} A_{j}\left(x, h D_{x^{\prime}}\right)\left(h D_{x_{1}}\right)^{j}\right\} .
\end{aligned}
$$

Suppose $\varphi \in \mathcal{C}^{\infty}\left(X, \Omega_{X}^{\frac{1}{2}}\right)$, and $x_{0} \in \partial X$. Using local coordinates at the boundary, we write $x_{0}=\left(0, x_{0}^{\prime}\right) \in\left\{x_{1} \geq 0\right\}$. Then $\varphi \in \mathcal{C}^{\infty}\left(X, \Omega_{X}^{\frac{1}{2}}\right)$ means there is a smooth extension $\tilde{\varphi}$ to an open neighbourhood of $x_{0} \in \mathbb{R}^{n}$. For a distribution $u \in \mathcal{D}^{\prime}\left(X, \Omega_{X}^{\frac{1}{2}}\right)$, we extend the notion of $\mathrm{WF}_{h}(u)$ to a neighbourhood of the boundary. We say $\left(x_{0}, \xi_{0}\right)=\left(0, x_{0}^{\prime}, \xi_{0}\right)$ is not in $\mathrm{WF}_{h}(u)$ if there is a product neighbourhood $\left(x_{0}, \xi_{0}\right) \in U \times V \subset \mathbb{R}^{2 n}$ and a normally differential operator $A \in \Psi_{h, d b}^{0,0}\left(U, \Omega_{U}^{\frac{1}{2}}\right)$ 
such that $\sigma_{h}(A)\left(x_{0}, \xi_{0}\right) \neq 0$ and

$$
A u \in h^{\infty} \mathcal{C}^{\infty}\left((0,1]_{h} ; \mathcal{C}^{\infty}\left(U, \Omega_{U}^{\frac{1}{2}}\right)\right) .
$$

Observe that if $u$ is smooth, then

$$
\left.\left(\mathrm{WF}_{h} u\right)\right|_{\partial X} \subset \mathrm{WF}_{h}\left(\left.u\right|_{\partial X}\right) \sqcup\left(\operatorname{ess-supp}_{h}\left(\left.u\right|_{\partial X}\right) \times N^{*}(\partial X)\right) .
$$

Similarly, using our identification of the $h$-wavefront set of a pseudodifferential operator as the essential support of its symbol, $A \in \Psi_{h, d b}^{0,0}$ with $\sigma_{h}(A) \neq 0$ at $\left(0, x_{0}^{\prime}, \xi_{0}\right)$ implies the $\xi_{1}$-direction is always contained in the $h$-wavefront set of $A$.

We are going to be interested in symbols which are compactly supported in $T^{*} X$, so we will need a notion of microlocal equivalence near the boundary which allows us to consider operators which are both normally differential and compactly supported in phase space. For this we return to our local coordinates at the boundary. Let $x_{0} \in \partial X, x_{0}=\left(0, x_{0}^{\prime}\right)$, and let $U \times V$ be a product neighbourhood of $\left(x_{0}, 0\right)$ in $\mathbb{R}^{2 n}$ such that $V$ is of the form $V=\left[-\epsilon_{0}, \epsilon_{0}\right]_{\xi_{1}} \times V_{\xi^{\prime}}$. By using the rescaling

$$
\left(x_{1}, \xi_{1}\right) \mapsto\left(x_{1} / \lambda, \lambda \xi_{1}\right),
$$

the ellipticity of $P$ outside a compact set implies $p \geq C^{-1}$ in

$$
U \times\left(\complement\left[-\epsilon_{0}, \epsilon_{0}\right]\right) \times V_{\xi^{\prime}} .
$$

Choose $\psi \in \mathcal{C}^{\infty}(\mathbb{R})$ satisfying

$$
\begin{aligned}
& \psi(t) \equiv 1 \text { for }|t| \leq \epsilon_{0}, \\
& \psi(t) \equiv 0 \text { for }|t| \geq 2 \epsilon_{0} .
\end{aligned}
$$

We say two semiclassically tempered operators $T$ and $T^{\prime}$ are microlocally equivalent near $(U \times V)^{2}$ if for all $A, A^{\prime} \in \Psi_{h, d b}^{0,0}$ satisfying

$$
\operatorname{proj}_{\left(x, \xi^{\prime}\right)}\left(\mathrm{WF}_{h} A\right) \text { is sufficiently close to } U \times V_{\xi^{\prime}},
$$

and similarly for $A^{\prime}$,

$$
\psi(P(h)) A\left(T-T^{\prime}\right) \psi(P(h)) A^{\prime}=\mathcal{O}\left(h^{\infty}\right): \mathcal{D}^{\prime}(X) \rightarrow \mathcal{C}^{\infty}(X) .
$$

In particular, if $A \in \Psi_{h . d b}^{0,0}$, we say $A$ is microlocally equivalent to

$$
\psi(P(h)) A
$$

and we will use this identification freely throughout.

3.2. Propagation of singularities. This section is basically a semiclassical adaptation of some of the propagation of singularities results at the boundary presented in [Hor3, Chap. 23], which in turn is strongly motivated by the Hadamard parametrix construction. According to [Hor3, App. C.5], under the noncharacteristic assumption we can find local symplectic coordinates near $Y$ so that $Y=\left\{x_{1}=0\right\}$ and (possibly after a sign change)

$$
p(x, \xi)=\xi_{1}^{2}-r\left(x, \xi^{\prime}\right), \quad \xi^{\prime}=\left(\xi_{2}, \ldots, \xi_{n}\right) .
$$

We define the hyperbolic set $H \subset T^{*} Y$ :

$$
H:=\left\{\left(x^{\prime}, \xi^{\prime}\right): r\left(0, x^{\prime}, \xi^{\prime}\right)>0\right\},
$$

on the lift of which the characteristic equation has the two roots $\left\{x_{1}=0, \xi_{1}=\right.$ $\left.\pm r\left(x, \xi^{\prime}\right)^{\frac{1}{2}}\right\}$. Thus the Hamiltonian vector field of $p$,

$$
H_{p}=2 \xi_{1} \partial_{x_{1}}-\partial_{\xi^{\prime}} r \partial_{x^{\prime}}+\partial_{x} r \partial_{\xi}
$$



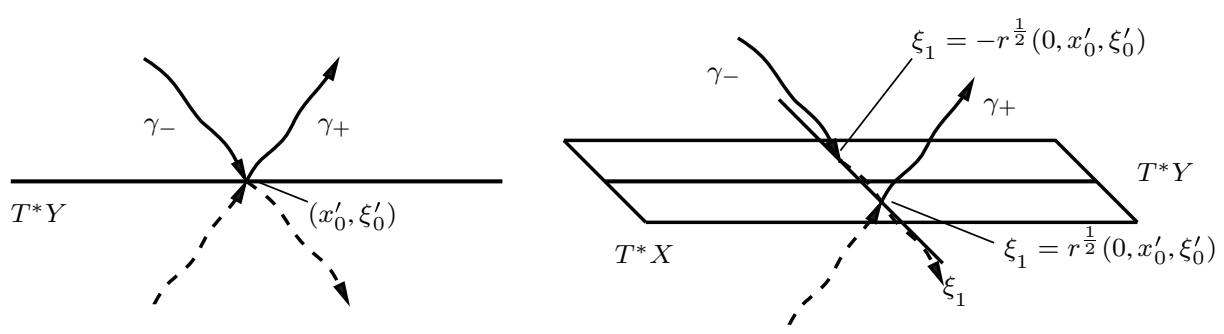

Figure 3. The incoming and outgoing bicharacteristics. Observe the reflection of $\gamma$ is continuous in a neighbourhood of $H$ in $T^{*} Y$ but not in $T^{*} X$, while the transmission of $\gamma$ is continuous in both.

points from $Y$ into $\left\{x_{1}>0\right\}$ or $\left\{x_{1}<0\right\}$, respectively, depending on which root of $r$ we choose. We call the corresponding bicharacteristic rays outgoing and incoming and write $\gamma_{+}$and $\gamma_{-}$, respectively (see Figure 3). We have the following factorization of the operator $P-z$ in our microlocal coordinates.

Lemma 3.1. There is a factorization of $P-z$ near $H$ :

$$
P-z=\left(h D_{1}-A_{-}\left(x, h D^{\prime}\right)\right)\left(h D_{1}-A_{+}\left(x, h D^{\prime}\right)\right)
$$

with $A_{ \pm} \in \Psi_{h, d b}^{1,0}$ having principal symbol $\pm r^{\frac{1}{2}}$.

Remark 3.2. We remark that $r\left(x, \xi^{\prime}\right)$ and $A_{ \pm}\left(x, h D^{\prime}\right)$ implicitly depend on the energy $z$, although we don't explicitly note this dependence where no ambiguity can arise.

Proof. We follow the proof for the $h$-independent version of the lemma found in Hor3, Lemma 23.2.8], although the original idea seems to be due to Hadamard. Using the coordinates above, the principal symbol of $P-z$ is (3.8). Set $A_{ \pm}^{1}=$ Op $\left( \pm r^{\frac{1}{2}}\right)$ so that

$$
P-z-\left(h D_{1}-A_{-}^{1}\right)\left(h D_{1}-A_{+}^{1}\right)=R_{1}(x, h D) \text { microlocally, }
$$

where $\sigma_{h} R_{1}=\mathcal{O}(h)$ is independent of $\xi_{1}$.

Now suppose we have $A_{ \pm}^{j}$ with principal symbols $\pm r^{\frac{1}{2}}$ such that

$$
P-z-\left(h D_{1}-A_{-}^{j}\right)\left(h D_{1}-A_{+}^{j}\right)=R_{j}(x, h D) \text { microlocally, }
$$

where $\sigma_{h} R_{j}=\mathcal{O}\left(h^{j}\right)$ is independent of $\xi_{1}$. Choose $a_{-}^{j}\left(x, \xi^{\prime}\right)=\mathcal{O}\left(h^{j}\right)$ satisfying

$$
\sigma_{h} R_{j}\left(x, \xi^{\prime}\right)+2 a_{-}^{j}\left(x, \xi^{\prime}\right) r^{\frac{1}{2}}\left(x, \xi^{\prime}\right)=0,
$$

which we can do since $r^{\frac{1}{2}}>0$ near $H$. We will similarly add $a_{+}^{j}\left(x, h D^{\prime}\right)$ to $A_{+}^{j}$, where $a_{+}^{j}=\mathcal{O}\left(h^{j}\right)$ is determined by the following calculation:

$$
\begin{aligned}
P-z & -\left(h D_{1}-A_{-}^{j}-a_{-}^{j}\left(x, h D^{\prime}\right)\right)\left(h D_{1}-A_{+}^{j}-a_{+}^{j}\left(x, h D^{\prime}\right)\right) \\
= & R_{j}(x, h D)-a_{-}^{j}\left(x, h D^{\prime}\right)\left(h D_{1}-A_{+}^{j}\right)-\left(h D_{1}-A_{-}^{j}\right)\left(a_{+}^{j}\left(x, h D^{\prime}\right)\right) \\
& +a_{-}^{j}\left(x, h D^{\prime}\right) a_{+}^{j}\left(x, h D^{\prime}\right) \text { microlocally. }
\end{aligned}
$$


On the level of principal symbols, this yields the requirement that

$$
\begin{aligned}
& \sigma_{h} R_{j}\left(x, \xi^{\prime}\right)-a_{-}^{j}\left(x, \xi^{\prime}\right)\left(\xi_{1}-r^{\frac{1}{2}}\left(x, \xi^{\prime}\right)\right)-\left(\xi_{1}+r^{\frac{1}{2}}\left(x, \xi^{\prime}\right)\right) a_{+}^{j}\left(x, \xi^{\prime}\right) \\
& \quad=-a_{-}^{j}\left(x, \xi^{\prime}\right)\left(\xi_{1}+r^{\frac{1}{2}}\left(x, \xi^{\prime}\right)\right)-\left(\xi_{1}+r^{\frac{1}{2}}\left(x, \xi^{\prime}\right)\right) a_{+}^{j}\left(x, \xi^{\prime}\right) \\
& \quad=0,
\end{aligned}
$$

which gives $a_{+}^{j}\left(x, \xi^{\prime}\right)=-a_{-}^{j}\left(x, \xi^{\prime}\right)$. By induction and Borel's Lemma the argument is complete.

We also have a microlocal factorization $P-z=\left(h D_{1}-\tilde{A}_{+}\right)\left(h D_{1}-\tilde{A}_{-}\right)$, where the principal symbols of $\tilde{A}_{ \pm}$are $\pm r^{\frac{1}{2}}$ as in the lemma. Suppose the $\gamma_{ \pm}$intersect $T^{*} Y$ at $\left(x_{0}^{\prime}, \xi_{0}^{\prime}\right)$. On $\gamma_{-}$we have $\xi_{1}=-r^{\frac{1}{2}}$, so $\left(h D_{1}-\tilde{A}_{+}\right)$is elliptic near $\gamma_{-}$. Then to solve (3.1), we need only solve $\left(h D_{1}-\tilde{A}_{-}\right) u=\left(h D_{1}-\tilde{A}_{+}\right)^{-1} f=\tilde{f}$.

Lemma 3.3. Suppose $u$ solves the following Cauchy problem in $\mathbb{R}_{+}^{n}$ :

$$
\left\{\begin{array}{l}
\left(h D_{1}-\tilde{A}_{-}\right) u=\tilde{f}, x_{1}>0 \\
\left.u\right|_{x_{1}=0}=\varphi\left(x^{\prime}\right)
\end{array}\right.
$$

Then

$$
\begin{aligned}
& \sup _{0 \leq y \leq T_{0}}\|u(y, \cdot)\|_{L_{x^{\prime}}^{2}\left(\mathbb{R}^{n-1} \times\left\{x_{1}=y\right\}\right)} \\
& \quad \leq C\|\varphi\|_{L_{x^{\prime}}^{2}\left(\mathbb{R}^{n-1} \times\left\{x_{1}=0\right\}\right)}+\frac{C_{T_{0}}}{h}\|\tilde{f}\|_{L^{1}\left(\left[0, T_{0}\right], L^{2}\left(\mathbb{R}^{n-1}\right)\right)} .
\end{aligned}
$$

Proof. Consider

$$
\begin{aligned}
\frac{1}{2} \partial_{y}\|u(y, \cdot)\|_{L^{2}\left(\mathbb{R}^{n-1} \times\left\{x_{1}=y\right\}\right)}^{2} & =\left\langle\partial_{y} u, u\right\rangle_{x^{\prime}} \\
& =-\frac{\operatorname{Im}}{h}\left\langle h D_{1} u, u\right\rangle_{x^{\prime}} \\
& \leq \frac{1}{h}\|u(y, \cdot)\|_{L_{x^{\prime}}^{2}}\|\tilde{f}(y, \cdot)\|_{L_{x^{\prime}}^{2}} \\
& \leq \frac{1}{4 h^{2}}\|\tilde{f}(y, \cdot)\|_{L_{x^{\prime}}^{2}}^{2}+\|u(y, \cdot)\|_{L_{x^{\prime}}^{2}}^{2}
\end{aligned}
$$

which by Gronwall's inequality gives the lemma.

Recall that the semiclassical Sobolev norms $\|\cdot\|_{H_{h}^{k}}$ are given by

$$
\|u\|_{H_{h}^{k}(V)}=\left(\sum_{|\alpha| \leq k} \int_{V}\left|(h D)^{\alpha} u\right|^{2} d x\right)^{\frac{1}{2}} .
$$

We observe that since $P$ is elliptic outside a compact set and $\left(h D_{1}-\tilde{A}_{+}\right)$is elliptic, replacing $\tilde{f}$ with $f$ and conjugating $\tilde{A}_{-}$above with the invertible operators $(C+$ $P)^{s / 2}$ for sufficiently large $C>0$, we can estimate the $L^{2}$-norm of $v=(C+P)^{s / 2} u$, and we get the Sobolev estimate

$$
\begin{aligned}
& \sup _{0 \leq y \leq T_{0}}\|u(y, \cdot)\|_{\left(H_{h}^{s}\right)_{x^{\prime}}\left(\mathbb{R}^{n-1} \times\left\{x_{1}=y\right\}\right)} \\
& \quad \leq C\|\varphi\|_{\left(H_{h}^{s}\right)_{x^{\prime}}\left(\mathbb{R}^{n-1} \times\left\{x_{1}=0\right\}\right)}+\frac{C_{T_{0}}\|f\|_{L^{1}\left(\left[0, T_{0}\right], H_{h}^{s}\left(\mathbb{R}^{n-1}\right)\right)} .}{h}
\end{aligned}
$$

We are interested in proving the existence of a microlocal solution propagator; hence we assume the wavefront set of $f$ is contained in a compact set $K$ in a 
neighbourhood of $\gamma_{-}$near $Y$. We assume as well that $K$ is contained in a single coordinate chart $U$ on which the assumptions of [SjZw1, Proposition 3.5] hold. Suppose $K \subset\left\{T_{1}<x_{1}<T_{2}\right\}$ and $U \subset\left\{T_{1}^{\prime}<x_{1}<T_{2}^{\prime}\right\}$.

Proposition 3.4. There are exactly two microlocal solutions $u_{i}, i=1,2$ to $\left(h D_{1}-\right.$ $\left.\tilde{A}_{-}\right) u=\tilde{f}$ microlocally near $\gamma_{-}$satisfying

$$
\begin{aligned}
& u_{1}=0 \text { microlocally for } x_{1} \leq T_{1}, \\
& u_{2}=0 \text { microlocally for } x_{1} \geq T_{2} .
\end{aligned}
$$

Proof. First we prove the proposition in a neighbourhood of $\mathrm{WF}_{h} \tilde{f}$. Let $\tilde{K}$ be the coordinate representation of $K$. Apply [SjZw1, Proposition 3.5] to write $\left(h D_{1}-\tilde{A}_{-}\right)$ as $h D_{1}$ in these coordinates. We write in the $x$-projection of this coordinate patch,

$$
\begin{aligned}
& u_{1}(x)=\frac{i}{h} \int_{-\infty}^{x_{1}} \tilde{f}\left(y, x^{\prime}\right) d y, \\
& u_{2}(x)=-\frac{i}{h} \int_{x_{1}}^{+\infty} \tilde{f}\left(y, x^{\prime}\right) d y,
\end{aligned}
$$

which satisfies $\left(h D_{1}-\tilde{A}_{-}\right) u=\tilde{f}$ with (3.11)-(3.12). Back in the original coordinates on our manifold, set $u_{1}=0$ for $x_{1} \leq T_{1}^{\prime}$ and $u_{2}=0$ for $x_{1} \geq T_{2}^{\prime}$. To continue, we will employ the energy estimates in Lemma 3.3. Suppose $u$ is a solution to $\left(h D_{1}-\tilde{A}_{-}\right) u=\tilde{f}$. Then for $v \in \mathcal{C}_{c}^{\infty}\left(\left[0, T_{0}\right) \times \mathbb{R}^{n-1}\right)$,

$$
\int_{0}^{T_{0}}\left\langle u,\left(h D_{1}-\tilde{A}_{-}\right) v\right\rangle_{x^{\prime}} d y=\int_{0}^{T_{0}}\langle\tilde{f}, v\rangle_{x^{\prime}} d y+\frac{h}{i}\langle u(0, \cdot), v(0, \cdot)\rangle_{x^{\prime}}
$$

since the Weyl quantizations of real symbols are selfadjoint. But from the proof of Lemma 3.3. replacing $y$ with $T_{0}-y$, we have since $\lim _{y \rightarrow T_{0}} v(y, \cdot)=0$,

$$
\sup _{0 \leq y \leq T_{0}}\|v(y, \cdot)\|_{L_{x^{\prime}}^{2}} \leq \frac{C}{h} \int_{0}^{T_{0}}\|g\|_{L_{x^{\prime}}^{2}} d y
$$

with $g=\left(h D_{1}-\tilde{A}_{-}\right) v$. We then have

$$
\left|\int_{0}^{T_{0}}\langle\tilde{f}, v\rangle_{x^{\prime}} d y+\frac{h}{i}\langle u(0, \cdot), v(0, \cdot)\rangle_{x^{\prime}}\right| \leq \frac{C_{\tilde{f}, u_{0, \cdot}}}{h} \int_{0}^{T_{0}}\|g\|_{L_{x^{\prime}}^{2}} d y .
$$

For $h>0$ we can extend to $g \in L^{2}$ the complex-conjugate linear form

$$
g \mapsto \int_{0}^{T_{0}}\langle\tilde{f}, v\rangle_{x^{\prime}} d y+\frac{h}{i}\langle u(0, \cdot), v(0, \cdot)\rangle_{x^{\prime}}
$$

by the Hahn-Banach Theorem. Thus by the Riesz Representation Theorem, for $\tilde{f} \in \mathcal{C}^{\infty}$ with sufficiently small wavefront set, we can find $u \in \mathcal{C}^{\infty}\left(\left[0, T_{0}\right), L_{x^{\prime}}^{2}\right)$ satisfying $\left(h D_{1}-\tilde{A}_{-}\right) u=\tilde{f}$.

For the uniqueness given by the conditions (3.11)-(3.12), note that if $f=0$ and $u(0, \cdot)=0$ in (3.10), then $u$ is zero. Replacing $x_{1}$ by $T_{0}-x_{1}$ we get the backwards uniqueness result.

Since $u_{1}$ is supported in the forward direction along the bicharacteristic $\gamma_{-}$, we refer to $u_{1}$ and $u_{2}$ as the forward and backward solutions, respectively. Let $u_{-}=u_{1}$ be the forward solution along the incoming bicharacteristic $\gamma_{-}$. So far we have proved the solution $u_{-}$satisfies $(P-z) u_{-}=f$ near $\gamma_{-}, u_{-}=0$ microlocally for 


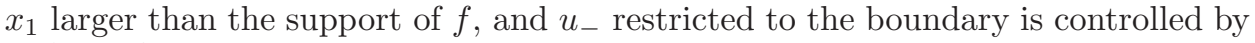
$h^{-1}$ in $L^{2}$ if the wavefront set of $f$ is sufficiently small.

The same energy method techniques can be used to solve the problem

$$
\left\{\begin{array}{l}
(P-z) u_{+}=0, \text { in } X \\
\left.u_{+}\right|_{Y}=\left.u_{-}\right|_{Y}
\end{array}\right.
$$

near $\gamma_{+}$so that $u=u_{-}-u_{+}$solves 3.1.

Corollary 3.5. If $f \in H_{h}^{\infty}$ has a sufficiently small wavefront set and $u$ solves (3.1), then

$$
u \in C^{1}\left(\left[0, T_{0}\right], H_{h}^{s}\left(\mathbb{R}^{n-1}\right)\right)
$$

for every $s$. In particular, $u(y, \cdot) \in \mathcal{C}^{\infty}\left(\mathbb{R}^{n-1} \times\left\{x_{1}=y\right\}\right)$ for each fixed $y \in\left[0, T_{0}\right]$.

In order to describe the propagation of singularities near the boundary, we first need the following lemma.

Lemma 3.6. Let $\gamma_{+}$be an interval on the outgoing bicharacteristic with one endpoint at $\left(0, x_{0}^{\prime}, r\left(0, x_{0}^{\prime}, \xi_{0}^{\prime}\right)^{\frac{1}{2}}, \xi_{0}^{\prime}\right)$. Then there is a pseudodifferential operator $Q\left(x, h D^{\prime}\right) \in \Psi_{h, d b}^{0,0}$ which satisfies

(i) $\sigma_{h}(Q)=0$ microlocally outside a neighbourhood of

$$
\left\{\left(x, \xi^{\prime}\right):\left(x, r\left(x, \xi^{\prime}\right)^{\frac{1}{2}}, \xi^{\prime}\right) \in \gamma_{+}\right\}
$$

(ii) $Q$ is noncharacteristic at $\left(x_{0}^{\prime}, \xi_{0}^{\prime}\right)$, and

(iii) $\left[Q\left(x, h D^{\prime}\right), h D_{1}-A_{+}\left(x, h D^{\prime}\right)\right]=0$ microlocally near $\gamma_{+}$.

Proof. The principal symbol of the commutator $\left[Q\left(x, h D^{\prime}\right), h D_{1}-A_{+}\left(x, h D^{\prime}\right)\right]$ is

$$
-i h\left\{\sigma_{h} Q\left(x, \xi^{\prime}\right), \xi_{1}-r^{\frac{1}{2}}\left(x, \xi^{\prime}\right)\right\}=i h\left(\partial_{x_{1}}-H_{r^{\frac{1}{2}}}\right) \sigma_{h} Q
$$

First we solve the Cauchy problem

$$
\left\{\begin{array}{l}
\left(\partial_{x_{1}}-H_{r^{\frac{1}{2}}}\right) Q_{0}=0, \quad(x, \xi) \in T^{*} X \\
Q_{0}=q_{0}, x_{1}=0
\end{array}\right.
$$

so that $Q_{0}$ is constant on orbits of the Hamiltonian system

$$
\left\{\begin{array}{l}
\dot{x}=-\partial_{\xi^{\prime}} b\left(x, \xi^{\prime}\right) \\
\dot{\xi}=\partial_{x^{\prime}} b\left(x, \xi^{\prime}\right)
\end{array}\right.
$$

where $\left({ }^{\cdot}\right):=\partial_{x_{1}}$ and $b\left(x, \xi^{\prime}\right):=r^{\frac{1}{2}}\left(x, \xi^{\prime}\right)$. Let $\chi_{x_{1}}(y, \eta)$ be a solution to (3.15) for initial conditions close to $\left(x_{0}^{\prime}, \xi_{0}^{\prime}\right)$ valid for $0 \leq x_{1} \leq T$, say. If $T>0$ is sufficiently small, then $\chi_{x_{1}}$ is invertible and $Q_{0}\left(x, \xi^{\prime}\right)=q_{0}\left(\chi_{x_{1}}^{-1}\left(x^{\prime}, \xi^{\prime}\right)\right)$ is the solution to (3.14). Now if we select $q_{0}$ compactly supported and $q_{0}=1$ in a neighbourhood of $\left(x_{0}^{\prime}, \xi_{0}^{\prime}\right)$, we satisfy conditions (i)-(ii) with

$$
\left[Q_{0}\left(x, h D^{\prime}\right), h D_{1}-A_{+}\left(x, h D^{\prime}\right)\right]=R_{1}\left(x, h D^{\prime}\right), \sigma_{h} R_{1}=\mathcal{O}\left(h^{2}\right),
$$

since $\xi_{1}$ only appears as a monomial of first order in the principal symbol. Now suppose we have $Q\left(x, h D^{\prime}\right)$ satisfying (i)-(ii) and

$$
\left[Q\left(x, h D^{\prime}\right), h D_{1}-A_{+}\left(x, h D^{\prime}\right)\right]=R_{j}\left(x, h D^{\prime}\right), \quad \sigma_{h} R_{j}=\mathcal{O}\left(h^{j+1}\right) .
$$

We solve the inhomogeneous Cauchy problem

$$
\left\{\begin{array}{l}
i h\left(\partial_{x_{1}}-H_{b}\right) Q_{j}\left(x, \xi^{\prime}\right)=-\sigma_{h} R_{j}\left(x, \xi^{\prime}\right), x \in T^{*} X \\
Q_{j}=q_{j}, x_{1}=0
\end{array}\right.
$$


for $Q_{j}=\mathcal{O}\left(h^{j}\right)$ and $q_{j}=\mathcal{O}\left(h^{j}\right)$, which we do by setting

$$
Q_{j}\left(x_{1}, \chi_{x_{1}}(y, \eta)\right)=q_{j}(y, \eta)+(i h)^{-1} \int_{0}^{x_{1}} \sigma_{h} R_{j}\left(s, \chi_{s}(y, \eta)\right) d s .
$$

Then $\tilde{Q}=Q+Q_{j}$ satisfies (i)-(ii) and

$$
\left[\tilde{Q}\left(x, h D^{\prime}\right), h D_{1}-A_{+}\left(x, h D^{\prime}\right)\right]=R_{j+1}\left(x, h D^{\prime}\right), \sigma_{h} R_{j+1}=\mathcal{O}\left(h^{j+2}\right) .
$$

By induction, the argument is finished, by setting $q_{j}=0$ for $j>0$.

Now suppose $\varphi \in \mathcal{C}_{c}^{\infty}\left(\mathbb{R}^{n-1}\right)$, where we identify $Y$ with $\mathbb{R}^{n-1}$ near $\left(x_{0}^{\prime}\right)$. Suppose further that $\left(x_{0}^{\prime}, \xi_{0}^{\prime}\right) \in T^{*}\left(\mathbb{R}^{n-1}\right) \backslash \mathrm{WF}_{h} \varphi$ and $\gamma_{+} \cap\left(x_{0}^{\prime}, \xi_{0}^{\prime}\right) \neq \emptyset$ as before. Choose $Q$ as in Lemma 3.6 so that

$$
\left[Q\left(x, h D^{\prime}\right), h D_{1}-A_{+}\left(x, h D^{\prime}\right)\right]=0
$$

microlocally and if $q$ is the principal symbol of $Q$, then $q\left(0, x_{0}^{\prime}, \xi_{0}^{\prime}\right) \neq 0$, but $q(0, \cdot, \cdot)$ vanishes outside a small neighbourhood of $\left(x_{0}^{\prime}, \xi_{0}^{\prime}\right)$. Thus $Q\left(x, h D^{\prime}\right) \varphi=0$ microlocally. Suppose $u_{+}$solves (3.13) with $\varphi$ replacing $\left.u_{-}\right|_{Y}$. Then $Q u_{+}$satisfies

$$
\left\{\begin{array}{l}
\left(h D_{1}-A_{+}\left(x, h D^{\prime}\right)\right) Q\left(x, h D^{\prime}\right) u_{+}=0, x_{1}>0 \\
Q\left(x, h D^{\prime}\right) u_{+}=0, x_{1}=0
\end{array}\right.
$$

microlocally. Hence by the energy estimate (3.10), $Q\left(x, h D^{\prime}\right) u_{+}=0$ microlocally. We conclude that $\mathrm{WF}_{h} u_{+} \subset \operatorname{char} q$.

We have proved the following proposition.

Proposition 3.7. With the notation as in the preceding paragraphs, $\mathrm{WF}_{h} u_{+} \subset$ $\chi_{x_{1}}\left(\mathrm{WF}_{h} \varphi\right)$ and $\mathrm{WF}_{h} u_{-} \subset \chi_{-x_{1}}\left(\mathrm{WF}_{h} \varphi\right)$. Further, as the $x_{1}$-direction is reversible, if at some $0<x_{1}<T_{0},\left(y^{\prime}, \eta^{\prime}\right) \in \mathrm{WF}_{h} u_{+}\left(x_{1}, \cdot\right)$, then $\chi_{-x_{1}}\left(y^{\prime}, \eta^{\prime}\right) \in \mathrm{WF}_{h} \varphi$ and $\chi_{-x_{1}-y}\left(y^{\prime}, \eta^{\prime}\right) \in \mathrm{WF}_{h} u_{-}(y, \cdot)$ for $0<y<T_{0}$. See Figure 4 .

In the special case that $Y=\partial X$ we have the following lemma to connect the notions of the $h$-wavefront set near $\gamma_{+}$and $\gamma_{-}$.

Lemma 3.8. Let $\left(x_{0}^{\prime}, \xi_{0}^{\prime}\right) \in H \cap \gamma$ be the reflection point at the boundary, let $\chi_{x_{1}}$ be a solution to (3.15) as above, and let $\varphi_{t}=\exp \left(t H_{p}\right)$. Then there is an odd diffeomorphism $t=t\left(x_{1}\right)$ and a function $\xi_{1}=\xi_{1}\left(x_{1}\right)$ such that $\left(x_{1}, \xi_{1} ; \chi_{x_{1}}\right)$ lies on $\gamma_{+}$and $\left(x_{1}, \xi_{1} ; \chi_{-x_{1}}\right)$ lies on $\gamma_{-}$for $x_{1}>0$ sufficiently small. That is, $\chi_{x_{1}}\left(x_{0}^{\prime}, \xi_{0}^{\prime}\right)$ coincides with the $\left(x^{\prime}, \xi^{\prime}\right)$ components of

$$
\varphi_{t}\left(0, x_{0}^{\prime}, r^{\frac{1}{2}}\left(0, x_{0}^{\prime}, \xi_{0}^{\prime}\right), \xi_{0}^{\prime}\right)
$$

Proof. Write $b\left(x, \xi^{\prime}\right)=r^{\frac{1}{2}}\left(x, \xi^{\prime}\right)$ as in the proof of Lemma 3.6] and note that $\chi_{-x_{1}}$ is the solution to (3.15) with $b$ replaced with $-b . \varphi_{t}$ satisfies the following differential equation on $\gamma_{+}$:

$$
\left\{\begin{array}{l}
\partial_{t} x_{1}=2 b \\
\partial_{t} x^{\prime}=-2 b b_{\xi^{\prime}} \\
\partial_{t} \xi_{1}=2 b b_{x_{1}} \\
\partial_{t} \xi^{\prime}=2 b b_{x^{\prime}} \\
x_{1}(0)=0 \\
x^{\prime}(0)=x_{0}^{\prime} \\
\xi_{1}(0)=b\left(0, x_{0}^{\prime}, \xi_{0}^{\prime}\right) \\
\xi^{\prime}(0)=\xi_{0}^{\prime} .
\end{array}\right.
$$




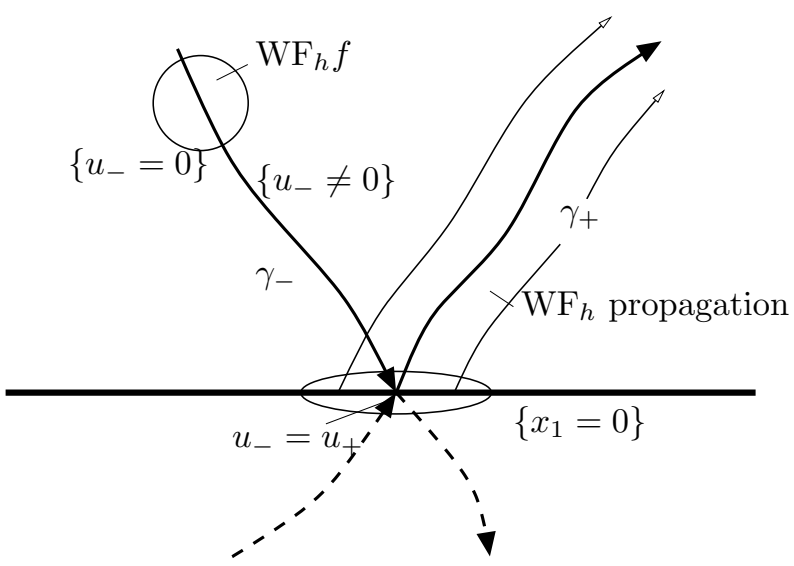

Figure 4. Proposition 3.7

Set $\left(y^{\prime}\left(x_{1}\right), \eta^{\prime}\left(x_{1}\right)\right)=\chi_{x_{1}}\left(x_{0}^{\prime}, \xi_{0}^{\prime}\right)$,

$$
t\left(x_{1}\right):=\int_{0}^{x_{1}}\left(2 b\left(y, y^{\prime}(y), \eta^{\prime}(y)\right)\right)^{-1} d y,
$$

and calculate

$$
\begin{aligned}
\frac{\partial}{\partial x_{1}} x^{\prime} & =\frac{\partial}{\partial t} x^{\prime} \frac{\partial t}{\partial x_{1}} \\
& =-b_{\xi^{\prime}}\left(x_{1}, x^{\prime}, \xi^{\prime}\right) \\
& =\frac{\partial}{\partial x_{1}} y^{\prime} .
\end{aligned}
$$

As $x^{\prime}$ and $y^{\prime}$ have the same initial conditions, we conclude that they are equal for sufficiently small $x_{1}$. For negative $t$, we define $t=t\left(x_{1}\right)$ in the incoming bicharacteristic to be the negative of that on the outgoing bicharacteristic, and a similar proof applies to $\chi_{-x_{1}}$.

Remark 3.9. With the addition of Lemma 3.8 we could write Proposition 3.7 in an equivalent form using $\exp \left(t H_{p}\right)$ in place of $\chi_{x_{1}}$. The important thing is that the wavefront set does not depend on $\xi_{1}$.

3.3. Microlocal propagator at the boundary. We now return to the special case where $Y=\partial X$. In the next section we will construct the Quantum Monodromy operator using the microlocal propagator. Suppose first that $X$ is a manifold without boundary. We define the forward and backward microlocal propagators of $P-z$, $I_{ \pm}^{z}(t)=\exp (\mp i t(P-z) / h)$, by the following evolution equation:

$$
\left\{\begin{array}{l}
h D_{t} I_{ \pm}^{z}(t) \pm(P-z) I_{ \pm}^{z}(t)=0, \\
I_{ \pm}^{z}(0)=\operatorname{id}_{L^{2} \rightarrow L^{2}} .
\end{array}\right.
$$

In the case of a manifold without boundary, this is a well-defined semigroup satisfying $\left[I_{ \pm}^{z}(t), P-z\right]=0$ and

$$
\mathrm{WF}_{h} I_{ \pm}^{z}(t) u \subset \exp \left( \pm t H_{p}\right)\left(\mathrm{WF}_{h} u\right) .
$$


We will show for $P \in \operatorname{Diff}_{h}^{2,0}$ with homogeneous principal symbol on a manifold with boundary, the microlocal propagator can be extended in a meaningful fashion as a family of microlocally defined $h$-FIOs with symbols which depend discontinuously on $t$ at points of reflection with the boundary, but still carry the commutator and wavefront set properties above.

Suppose $\gamma$ reflects off $\partial X$ at the points

$$
m_{ \pm}:=\left(0, x_{0}^{\prime}, \pm r^{\frac{1}{2}}\left(0, x_{0}^{\prime}, \xi_{0}^{\prime}\right), \xi_{0}^{\prime}\right)
$$

with the incoming and outgoing rays, $\gamma_{\mp}$, intersecting $\partial X$ at $m_{\mp}$, respectively. Since $p$ is assumed smooth up to the boundary, we may extend $p$ and $\gamma_{-}$to a neighbourhood of $m_{-}$in $\left\{x_{1} \leq 0\right\}$. We will show that the functions $v\left(x^{\prime}\right)$ defined on $\partial X$ can be identified with the microlocal kernel of $P-z$ in a neighbourhood of $m_{-}$. We factorize $P-z$ as in Lemma 3.1. $P-z=\left(h D_{1}-\tilde{A}_{+}\right)\left(h D_{1}-\tilde{A}_{-}\right)$ microlocally near $m_{-}$. Near $\gamma_{-}$the operator $\left(h D_{1}-\tilde{A}_{+}\right)$is elliptic. Thus we want to be able to solve

$$
\left\{\begin{array}{l}
\left(h D_{1}-\tilde{A}_{-}\right) u=0, \quad x_{1}>0 \\
u\left(0, x^{\prime}\right)=v\left(x^{\prime}\right), \quad x_{1}=0
\end{array}\right.
$$

for any boundary condition $v$, microlocally near $m_{-}$. The following proposition is a semiclassical adaptation of the geometric optics construction (see, for example, Tay1, §6.6] and [Hor3, §17.4]; the proof in the semiclassical case can be found in, for instance, $\mathrm{EvZw})$.

Proposition 3.10. There is a microlocal solution to (3.17) given by the oscillatory integral

$$
u(x)=\frac{1}{(2 \pi h)^{n-1}} \int e^{i / h\left(\varphi\left(x, \xi^{\prime}\right)-\left\langle y^{\prime}, \xi^{\prime}\right\rangle\right)} b\left(x, \xi^{\prime}\right) v\left(y^{\prime}\right) d \xi^{\prime} d y^{\prime},
$$

where $b\left(0, x^{\prime}, \xi^{\prime}\right)=1$ microlocally near $\left(x_{0}^{\prime}, \xi_{0}^{\prime}\right)$ and $\varphi$ solves the eikonal equation

$$
\left\{\begin{array}{l}
\partial_{x_{1}} \varphi\left(x, \xi^{\prime}\right)-a\left(x, \partial_{x^{\prime}} \varphi\left(x, \xi^{\prime}\right)\right)=0, x_{1}>0 \\
\varphi\left(0, x^{\prime}, \xi^{\prime}\right)=\left\langle x^{\prime}, \xi^{\prime}\right\rangle, x_{1}=0
\end{array}\right.
$$

with $a=\sigma_{h}\left(\tilde{A}_{-}\right)$. Further, $u(x)$ is unique microlocally.

Proposition 3.11. Let $X$ be a manifold with boundary, $P \in \operatorname{Diff}_{h}^{2,0}$ be a differential operator with homogeneous principal symbol $p$, and assume $\partial X$ is noncharacteristic with respect to $p$. Let $U_{ \pm} \subset T^{*} X$ be a neighbourhood of $m_{ \pm} \in T^{*} X$, and assume $P-z$ and $p-z$ are factorized near the boundary as in Lemma 3.1 and equation (3.8), respectively.

(i) For each $m_{0} \in \gamma_{-} \cap U_{-}$sufficiently close to $m_{-}$, and $z \in\left[-\epsilon_{0}, \epsilon_{0}\right]$ for $\epsilon_{0}>0$ sufficiently small, there exist $h$-FIOs, $I_{ \pm}^{z}(t)$, defined microlocally near

$$
\exp \left( \pm t H_{p}\right)\left(m_{0}\right) \times m_{0}
$$

satisfying

$$
\left\{\begin{array}{l}
h D_{t} I_{ \pm}^{z}(t) \pm(P-z)(t) I_{ \pm}^{z}(t)=0 \\
I_{ \pm}^{z}(0)=\operatorname{id}_{L^{2} \rightarrow L^{2}}
\end{array}\right.
$$

for $t \neq t_{1}$, where $m_{-}=\exp \left(t_{1} H_{p}\right)\left(m_{0}\right)$. 
(ii) We have $\left[(P-z)(t), I_{ \pm}^{z}(t)\right]=0$ for all $t \neq t_{1}$ sufficiently small, and if $u(x) \in L^{2}$ is a microlocal solution to

$$
\left\{\begin{array}{l}
(P-z) u=f \in L^{2}, x \in \stackrel{\circ}{X}, \\
u=0, x \in \partial X
\end{array}\right.
$$

near $m_{0}$, then $I_{ \pm}^{z}(t) u(x)$ is a microlocal solution to (3.21) near $\exp \left( \pm t H_{p}\right)\left(m_{0}\right)$.

(iii) If $\mathrm{WF}_{h} u \subset K$, where $K$ is a compact neighbourhood of a point $m_{0}$, then

$$
\mathrm{WF}_{h} I_{ \pm}^{z}(t) u \subset \exp \left( \pm t H_{p}\right)(K) .
$$

Proof. Fix $m_{0}$. According to [SjZw1, Proposition 3.5], $P-z$ may be conjugated to $h D_{x_{1}}$ in a neighbourhood of $m_{0}$. Then we use the proof of Proposition 3.4 to find a solution $u_{-, 1}$ to $(P-z) u=f$ near $m_{0}$. Use the microlocal forward propagator defined for a neighbourhood of $\gamma_{-}$extended to a neighbourhood of $m_{-}$to define $u_{-, 1}$ along $\gamma_{-}$. That is, $I_{+}^{z}(t) u_{-, 1}$ satisfies

$$
(P-z) I_{+}^{z}(t) u_{-, 1}=f
$$

microlocally near $\exp \left(t H_{p}\right)\left(m_{0}\right), 0 \leq t \leq t_{1}$. Let $v_{-}\left(x^{\prime}\right)=\left.I\left(t_{1}\right) u_{-, 1}\right|_{\partial X}$, and use Proposition 3.10 to find a function $u_{-, 2}$ satisfying

$$
\left\{\begin{array}{l}
(P-z) u_{-, 2}=0 \\
u_{-, 2} \mid \partial X=v_{-}\left(x^{\prime}\right)
\end{array}\right.
$$

microlocally near $m_{-}$. Let

$$
u_{-}=u_{-, 1}-I_{+}^{z}\left(-t_{1}\right) u_{-, 2},
$$

so that $I_{+}^{z}(t) u_{-}$satisfies (3.21) microlocally near $\exp \left(t H_{p}\right)\left(m_{0}\right), 0 \leq t<t_{1}$.

Fix $m_{2}=\exp \left(t_{2} H_{p}\right)\left(m_{0}\right) \in \gamma_{+}$sufficiently close to $m_{+}$so that we can similarly construct $I_{+}^{z}\left(t_{2}-t\right) u_{+}$satisfying (3.21) microlocally near $\exp \left(-t H_{p}\right)\left(m_{2}\right)$ for $0 \leq$ $t<t_{2}-t_{1}$. We extend $I_{+}^{z}(t)$ to be discontinuous at $t_{1}$, so that if $u$ solves (3.21) microlocally near $m_{0}$, then

$$
I_{+}^{z}\left(t_{1}\right) u=u_{-}+u_{+}
$$

with

$$
\mathrm{WF}_{h} u_{ \pm} \subset U_{ \pm} \cap\left\{x_{1} \geq 0\right\} .
$$

We need to verify that this extension of $I_{+}^{z}(t)$ satisfies (i), (ii), and (iii). For $0 \leq t<t_{1}$ this is clear because $I_{+}^{z}(t)$ is the usual semigroup. At $t_{1}$, we have

$$
\begin{aligned}
(P-z) I_{+}^{z}\left(t_{1}\right) u & =(P-z)\left(I_{+}^{z}\left(t_{1}\right) u_{-}+I_{+}^{z}\left(t_{2}-t_{1}\right) u_{+}\right) \\
& =f_{-}+f_{+},
\end{aligned}
$$

with $f_{ \pm}=f$ microlocally near $m_{ \pm}$. Thus (ii) and (iii) are clear.

For (i), let $A=\mathrm{Op}_{h}^{w}(a)$ be a symbol defined microlocally in a neighbourhood of $\exp \left(t H_{p}\right)\left(m_{0}\right)$. Assume $m_{ \pm} \notin \mathrm{WF}_{h} A$, and let $B=\mathrm{Op}_{h}^{w}\left(\exp \left(t H_{p}\right)^{*} a\right)$. Then

$$
A I_{+}^{z}(t) u=I_{+}^{z}(t) B u
$$

microlocally, and by [SjZw1, Lemma 3.4] (see also [EvZw), $I^{z}(t)$ satisfies (3.20).

The proof for $I_{-}^{z}(t)$ is similar. 
Corollary 3.12. Let $X, P$, and $p$ be as in Proposition 3.11. Suppose $\gamma(t)$ is a periodic orbit for $\exp \left(t H_{p}\right)$ of period $T$ which has a finite number of transversal reflections off $\partial X$. Then for any $m \in \gamma(t), \pi(m) \cap \partial X=\emptyset$, where $\pi: T^{*} X \rightarrow X$ is the projection, and there exist $h$-FIOs, $I_{ \pm}^{z}(t)$, defined microlocally near $\exp \left(t H_{p}\right)(m) \times m$ for $0 \leq|t| \leq T$ satisfying

(i)

$$
\left\{\begin{array}{l}
h D_{t} I_{ \pm}^{z}(t) \pm(P-z) I_{ \pm}^{z}(t)=0 \\
I_{ \pm}^{z}(0)=\operatorname{id}_{L^{2} \rightarrow L^{2}}
\end{array}\right.
$$

for almost every $t$.

(ii) $\left[P-z, I_{ \pm}^{z}(t)\right]=0$, and if $u(x) \in L^{2}$ satisfies $(P-z) u=f \in L^{2}$ microlocally near $m$, then $I_{ \pm}^{z}(t) u(x)$ satisfies

$$
(P-z) I_{ \pm}^{z}(t) u(x)=f(x)
$$

microlocally near $\exp \left( \pm t H_{p}\right)(m)$.

(iii) If $\mathrm{WF}_{h} u \subset K$, where $K$ is a compact neighbourhood of a point $m$, then

$$
\mathrm{WF}_{h} I_{ \pm}^{z}(t) u \subset \exp \left( \pm t H_{p}\right)(K) .
$$

Proof. This follows immediately from Proposition 3.11 and the uniqueness of solutions to ordinary differential equations.

\section{Quantum Monodromy construction}

In this section, we construct the Quantum Monodromy operator

$$
M(z): L^{2}\left(\mathbb{R}^{n-1}\right) \rightarrow L^{2}\left(\mathbb{R}^{n-1}\right)
$$

and prove some basic properties. Here we follow $[\mathrm{SjZw1}$ and the somewhat simplified presentation in [SjZw2. It is classical (see, for example, [AbMa, Theorem 28.5]) that the assumptions on $p$ imply there exists $\epsilon_{0}>0$ such that for $-2 \epsilon_{0} \leq E \leq 2 \epsilon_{0}$ there is a closed semi-hyperbolic orbit in the level set $\{p=E\}$. Let us assume $E=0$ for simplicity, and let $z \in\left[-\epsilon_{0}, \epsilon_{0}\right] \subset \mathbb{R}$. Then $p-z$ is the principal symbol of $P-z$, and $p-z$ admits a closed semi-hyperbolic orbit in the level set $\{p-z=0\}$, say, $\gamma(z)$ of period $T(z)$.

We work microlocally in a neighbourhood of

$$
\Gamma:=\bigcup_{-\epsilon_{0} \leq z \leq \epsilon_{0}} \gamma(z) \subset T^{*} X .
$$

Fix $m_{0}(z) \in \gamma(z), m_{0}(z) \cap \partial X=\emptyset$, depending smoothly on $z$, and set

$$
m_{1}(z)=\exp \left(\frac{1}{2} T(z) H_{p}\right)\left(m_{0}(z)\right) .
$$

By perturbing $m_{0}(z)$ and shrinking $\epsilon_{0}>0$ if necessary, we may assume $\pi\left(m_{1}(z)\right) \cap$ $\partial X=\emptyset$ as well. Assume we are working with a fixed $z$. Define

$$
\begin{array}{r}
\operatorname{ker}_{m_{0}(z)}(P-z)=\left\{u \in L^{2}\left(\operatorname{neigh}\left(m_{0}(z)\right)\right):(P-z) u=0\right. \\
\text { microlocally near } \left.m_{0}(z)\right\},
\end{array}
$$

where "neigh $\left(m_{0}(z)\right)$ " refers to a germ, or a small arbitrary neighbourhood of $m_{0}(z)$ which is allowed to change from line to line. Similarly we have

$$
\begin{array}{r}
\operatorname{ker}_{m_{1}(z)}(P-z)=\left\{u \in L^{2}\left(\text { neigh }\left(m_{1}(z)\right)\right):(P-z) u=0\right. \\
\text { microlocally near } \left.m_{1}(z)\right\} .
\end{array}
$$


We define the forward and backward microlocal propagators $I_{ \pm}^{z}$ as in Corollary 3.12, Then

$$
I_{+}^{z}(t): \operatorname{ker}_{m_{0}(z)}(P-z) \rightarrow \operatorname{ker}_{\exp \left(t H_{p}\right)\left(m_{0}(z)\right)}(P-z),
$$

and since

$$
\exp \left(T(z) H_{p}\right)\left(m_{0}(z)\right)=m_{0}(z)
$$

we define the Absolute Quantum Monodromy operator

$$
\mathcal{M}(z): \operatorname{ker}_{m_{0}(z)}(P-z) \rightarrow \operatorname{ker}_{m_{0}(z)}(P-z)
$$

by

$$
\mathcal{M}(z):=I_{+}(T(z))
$$

It is convenient to introduce an inner product structure on $\operatorname{ker}_{m_{0}(z)}(P-z)$ (see HeSj]). For this, let $\chi \in \mathcal{C}^{\infty}\left(T^{*} X\right)$ be a microlocal cutoff supported near $\gamma(z)$ satisfying the following properties (see Figure 6):

$$
\begin{aligned}
& \chi \equiv 1 \text { on } \exp \left(t H_{p}\right)\left(m_{0}(z)\right) \text { for } 0 \leq t \leq \frac{1}{2} T(0), \\
& \chi \equiv 0 \text { on } \exp \left(t H_{p}\right)\left(m_{0}(z)\right) \text { for } \frac{1}{2} T(0)+\delta \leq t \leq T(0)-\delta, \delta>0 .
\end{aligned}
$$

Let $[P, \chi]_{+}$denote the part of the commutator supported near $m_{0}(z)$, where we use $\chi$ to denote both the function and the quantization whenever unambiguous, and for $u, v \in \operatorname{ker}_{m_{0}(z)}(P-z)$, define the Quantum Flux product as

$$
\langle u, v\rangle_{Q F}:=\left\langle\frac{i}{h}[P, \chi]_{+} u, v\right\rangle_{L^{2}\left(\operatorname{neigh}\left(m_{0}(z)\right)\right)} .
$$

According to [SjZw1, Proposition 3.5] (see also Hor3, 21.3.1] for the classical case), there is a neighbourhood of $m_{0}(z)$ and an $h$-Fourier integral operator $F$ defined microlocally near $m_{0}(0)$ such that $F(P-z) F^{-1}=h D_{x_{1}}$ on $L^{2}(\widetilde{V})$, where $\widetilde{V} \subset \mathbb{R}^{n}$ is an open neighbourhood of $0 \in \mathbb{R}^{n}$. Then $\operatorname{ker}_{m_{0}(z)}(P-z)$ can be identified with $L^{2}(V)$, where $V \subset \mathbb{R}^{n-1}$ is an open neighbourhood of $0 \in \mathbb{R}^{n-1}$. Let

$$
K(z): L^{2}(V) \longleftrightarrow \operatorname{ker}_{m_{0}(z)}(P-z)
$$

be the identification, and define the adjoint $K(z)^{*}$ with respect to the $L^{2}$ inner product on $\operatorname{ker}_{m_{0}(z)}(P-z)$. Note that

$$
K(z)^{*}: \operatorname{ker}_{m_{0}(z)}(P-z) \longleftrightarrow L^{2}(V)
$$

is an identification as well. The following two lemmas are from [SjZw1.

Lemma 4.1. The operator

$$
U:=K(z)^{*} \frac{i}{h}[P, \chi]_{+} K(z): L^{2}(V) \rightarrow L^{2}(V)
$$

is positive definite. Setting $\widetilde{K}(z)=K(z) U^{\frac{1}{2}}$, we have

$$
\widetilde{K}(z)^{*} \frac{i}{h}[P, \chi]_{+} \widetilde{K}(z)=\mathrm{id}: L^{2}(V) \rightarrow L^{2}(V) .
$$


Proof. Using [SjZw1, Proposition 3.5], we write

$$
\begin{aligned}
\left\langle K(z) * \frac{i}{h}[P, \chi]_{+} K(z) v, v\right\rangle_{L^{2}(V)} & =\left\langle\partial_{x_{1}} \chi K(z) v, K(z) v\right\rangle_{L^{2}\left(\operatorname{neigh}\left(m_{0}(z)\right)\right)} \\
& \geq C^{-1}\|v\|^{2} .
\end{aligned}
$$

Remark 4.2. In light of Lemma 4.1, we replace $K(z)$ with $\widetilde{K}(z)$ and write

$$
K(z)^{-1}=K(z)^{*} \frac{i}{h}[P, \chi]_{+} \cdot
$$

Lemma 4.3. The Quantum Flux product $\langle\cdot, \cdot\rangle_{Q F}$ does not depend on the choice of $\chi$ satisfying (4.3) (4.4). In addition, $\mathcal{M}(z)$ is unitary on $\operatorname{ker}_{m_{0}(z)}(P-z)$ with respect to this product.

Proof. Suppose $u, v \in L^{2}(V)$ and suppose $\tilde{\chi}$ is another function satisfying (4.3)(4.4) which agrees with $\chi$ near $m_{1}(z)$. Then $[P, \tilde{\chi}-\chi]_{+}=[P, \tilde{\chi}-\chi],(P-z) K(z) u=$ 0 , and $K(z)^{*}(P-z)=((P-z) K(z))^{*}$ imply

$$
\left\langle\frac{i}{h}[P, \tilde{\chi}-\chi]_{+} K(z) u, K(z) v\right\rangle=\left\langle\frac{i}{h}(\widetilde{\chi}-\chi) K(z) u,(P-z) K(z) v\right\rangle=0 .
$$

To see that $\mathcal{M}(z)$ is unitary, observe for $\tilde{u} \in \operatorname{ker}_{m_{0}(z)}(P-z)$,

$$
\begin{aligned}
\left\langle\frac{i}{h}\right. & {\left.[P, \chi]_{+} I_{+}^{z}(T(z)) \tilde{u}, I_{+}^{z}(T(z)) \tilde{u}\right\rangle } \\
& =\left\langle\frac{i}{h}\left[P, I_{-}^{z}(T(z)) \chi I_{+}^{z}(T(z))\right]_{+} \tilde{u}, \tilde{u}\right\rangle \\
& =\left\langle\frac{i}{h}[P, \tilde{\chi}]_{+} \tilde{u}, \tilde{u}\right\rangle,
\end{aligned}
$$

where $\tilde{\chi}=\exp \left(T H_{p}\right)^{*} \chi$ satisfies (4.3)-(4.4).

Next we restrict our attention to $L^{2}(V)$ by defining the Quantum Monodromy operator $M(z): L^{2}(V) \rightarrow L^{2}(V)$ by

$$
M(z)=K(z)^{-1} \mathcal{M}(z) K(z) .
$$

Lemma 4.4. $M(z): L^{2}(V) \rightarrow L^{2}(V)$ is unitary, and $M(z)$ is a (microlocally unitary) quantization of the Poincaré map $S$.

Proof. Let $u \in L^{2}(V)$. We calculate:

$$
\begin{aligned}
\langle M(z) u, M(z) u\rangle_{L^{2}(V)} & \\
& =\left\langle K(z)^{-1} \mathcal{M}(z) K(z) u, K(z)^{-1} \mathcal{M}(z) K(z) u\right\rangle_{L^{2}(V)} \\
& =\left\langle\left(K(z)^{*}\right)^{-1} K(z)^{-1} \mathcal{M}(z) K(z) u, \mathcal{M}(z) K(z) u\right\rangle_{L^{2}\left(\operatorname{neigh}\left(m_{0}(z)\right)\right)} \\
& =\left\langle\frac{i}{h}[P, \chi]_{+} \mathcal{M}(z) K(z) u, \mathcal{M}(z) K(z) u\right\rangle_{L^{2}\left(\operatorname{neigh}\left(m_{0}(z)\right)\right)} \\
& =\langle K(z) u, K(z) u\rangle_{L^{2}\left(\operatorname{neigh}\left(m_{0}(z)\right)\right)} \\
& =\langle u, u\rangle_{L^{2}(V)} .
\end{aligned}
$$

In order to prove $M(z)$ is the quantization of the Poincaré map, we will use SjZw1, Lemma 3.4]. We need to prove for pseudodifferential operators $A, B \in \psi_{h}^{0,0}(V)$ such 
that $\sigma_{h}(B)=S^{*} \sigma_{h}(A)$, that we have $A M(z)=M(z) B$. Without loss of generality, we write $x^{\prime}=\left(x_{2}, \ldots, x_{n}\right) \in V$ for the variables in $V$ and $x=\left(x_{1}, x^{\prime}\right) \in \operatorname{neigh}(\gamma(z))$ for the variables in $X$ near $\gamma$. Then for $v \in L^{2}(V) \cap \mathcal{C}^{\infty}(V)$,

$$
\begin{aligned}
& M(z) B\left(x^{\prime}, h D_{x^{\prime}}\right) v\left(x^{\prime}\right) \\
& \quad=K(z)^{-1} \mathcal{M}(z) K(z) B\left(x^{\prime}, h D_{x^{\prime}}\right) v\left(x^{\prime}\right) \\
& \quad=K(z)^{-1} I_{+}^{z}(T(z)) B\left(x^{\prime}, h D_{x^{\prime}}\right) I_{-}^{z}(T(z)) I_{+}^{z}(T(z)) K(z) v\left(x^{\prime}\right) \\
& \quad=K(z)^{-1} \mathrm{Op}\left(\left(\exp \left(T H_{p}\right)\right)^{*} \sigma_{h}(B)\right)\left(x, h D_{x}\right) I_{+}^{z}(T(z)) K(z) v\left(x^{\prime}\right) \\
& =A\left(x^{\prime}, h D_{x^{\prime}}\right) M(z) v\left(x^{\prime}\right) .
\end{aligned}
$$

\section{The Grushin Problem}

5.1. Motivation of the Grushin problem. In this section we follow SjZw1 and show how the Quantum Monodromy operator arises naturally in the context of a Grushin problem near $\gamma$. This is a generalization of the linear algebra Grushin problem: Suppose

$$
\begin{aligned}
A & : \quad H \rightarrow H, \\
B & : \quad H_{-} \rightarrow H, \\
C & : \quad H \rightarrow H_{+}, \text {and } \\
D & : \quad H_{-} \rightarrow H_{+}
\end{aligned}
$$

are matrices acting on finite-dimensional Hilbert spaces $H, H_{-}$, and $H_{+}$, and

$$
\left(\begin{array}{ll}
\alpha & \beta \\
\sigma & \delta
\end{array}\right)=\left(\begin{array}{ll}
A & B \\
C & D
\end{array}\right)^{-1}
$$

where

$$
\begin{aligned}
\alpha & : H \rightarrow H, \\
\beta & : \quad H_{+} \rightarrow H, \\
\sigma & : \quad H \rightarrow H_{-}, \text {and } \\
\delta & : \quad H_{+} \rightarrow H_{-} .
\end{aligned}
$$

Then $A$ is invertible if and only if $\delta$ is invertible, in which case

$$
A^{-1}=\alpha-\beta \delta^{-1} \sigma .
$$

It appears counterintuitive at first that understanding the invertibility of a larger matrix might be easier than understanding the invertibility of a submatrix. However, when the entries are operators instead of matrices, the situation may change. In the next section we will see that introducing a matrix of operators will allow us to understand the microlocal invertibility of $P-z$ near a periodic orbit by constructing a parametrix in the double cover of a neighbourhood of the orbit.

5.2. The Grushin problem reduction. Throughout this section, we suppress the dependence on $z$ whenever unambiguous for ease in exposition. We will build operators $R_{+}=R_{+}(z): \mathcal{D}^{\prime}(X) \rightarrow \mathcal{D}^{\prime}(V)$ and $R_{-}=R_{-}(z): \mathcal{D}^{\prime}(V) \rightarrow \mathcal{D}^{\prime}(X)$ such 
that

$$
\mathcal{P}:=\left(\begin{array}{cc}
\frac{i}{h}(P-z) & R_{-} \\
R_{+} & 0
\end{array}\right): \mathcal{D}^{\prime}(X) \times \mathcal{D}^{\prime}(V) \rightarrow \mathcal{D}^{\prime}(X) \times \mathcal{D}^{\prime}(V)
$$

has microlocal inverse

$$
\mathcal{E}=\left(\begin{array}{cc}
E & E_{+} \\
E_{-} & E_{-+}
\end{array}\right)
$$

near $\gamma \times(0,0)$, where $E, E_{+}$, and $E_{-}$will be defined later, and

$$
E_{-+}=I-M(z)
$$

Let us briefly explain the intuitive idea behind the construction of $\mathcal{P}$ and $\mathcal{E}$. The method of construction will be to build the operators in $\mathcal{P}$ and $\mathcal{E}$ simultaneously. We first construct $R_{+}(z)$ as a microlocal Cauchy data operator. That is, $R_{+}(z)$ takes a solution $u$ of $(P-z) u=0$ to its Cauchy data microlocally restricted to the Poincaré section and identifies this with a phase space restriction in $T^{*} V$ via the operator $K^{*}(z)$. The operator $R_{-}(z)$ intuitively does the reverse by taking a distribution $v$ on $V$ and first identifying $v$ with the distribution on $X$ microlocally in the kernel of $P-z$ and then extending it to a microlocal solution in a tubular neighbourhood of $\gamma(z)$. However, in order to be a microlocal solution in a whole neighbourhood of $\gamma(z)$, the germ of the solution must agree when propagated around the orbit. In general by simply propagating around $\gamma(z)$ we do not expect the germs to agree, but it turns out by cutting and gluing that we can construct a microlocal solution at least on the double cover space. To accomplish this, we propagate forward and backward halfway around $\gamma(z)$ and compare the resulting germs using the operator $E_{-+}(z)$ (and hence the quantum monodromy operator). The operator $E_{+}(z)$ is constructed by propagating almost halfway forward and backward around $\gamma(z)$ to get a microlocal solution away from the point $m_{1}(z)$ defined in (4.1). If we blindly try to glue together forward and backward solutions, we get an error term which can be eliminated if we also know how to solve the inhomogeneous problem. By lifting to the double cover space, we can construct forward and backward microlocal solutions to the inhomogeneous problem all the way around $\gamma(z)$, which we glue together using an auxiliary operator $E_{0}(z)$. The constraint $\mathcal{P}(z) \mathcal{E}(z)=$ id then yields two more equations, and $E$ and $E_{-}$are constructed from the previously constructed operators to satisfy these constraints.

The following construction of the solution to the Grushin problem is from SjZw1, with the addition here that we allow $\gamma(z)$ to reflect transversally off the boundary of $\partial X$. Recall that $\chi \in \mathcal{C}_{c}^{\infty}\left(T^{*} X\right)$ satisfies (4.3)-(4.4), and begin by setting

$$
R_{+}=K^{*} \frac{i}{h}[P, \chi]_{+}
$$

Then if $u$ satisfies $(P-z) u=0$ microlocally near $m_{0}(z), R_{+} u$ is the microlocal Cauchy data. That is, for $v \in L^{2}(V), u=K v$ is a solution to the microlocal Cauchy problem

$$
\left\{\begin{array}{c}
(P-z) u=0 \\
R_{+} u=v
\end{array}\right.
$$


near $\gamma \times(0,0)$. To construct a global solution, let $K_{f}(t):=I_{+}(t) K$ and $K_{b}(t):=$ $I_{-}(t) K$ be the forward and backward (respectively) Cauchy problem solution operators. Note for $t \sim T / 2$ that we have

$$
\begin{aligned}
K_{f}(t) & =I_{+}(t) K \\
& =I_{-}(t) K K^{-1} \mathcal{M}(z) K \\
& =K_{b}(t) M(z),
\end{aligned}
$$

so microlocally near $m_{1} \times(0,0)$ we have $K_{f}=K_{b} M(z)$, where $m_{1}$ is defined in (4.1). Now for $\Omega$ a neighbourhood of $\gamma$, we can solve (5.2) in $\Omega \backslash$ neigh $\left(m_{1}\right)$. To do this, set

$$
E_{+} v=\chi K_{f} v+(1-\chi) K_{b} v
$$

so $E_{+} v$ satisfies

i) $\quad E_{+} v=K v$ in a neighbourhood of $m_{0}(z)$;

ii) $\quad R_{+} E_{+}=$id microlocally near $(0,0) \times(0,0) \in\left(T^{*} V\right)^{2}$.

With $[\cdot, \cdot]_{-}$denoting the part of the commutator supported near $m_{1}(z)$, we calculate:

$$
(P-z) E_{+} v=[P, \chi]_{-} K_{f} v-[P, \chi]_{-} K_{b} v,
$$

since $K_{f}=K_{b}$ microlocally near $m_{0}(z) \times(0,0)$ and $(P-z) I_{ \pm}(t) K v=0$ microlocally near $\exp \left(t H_{p}\right)\left(m_{0}(z)\right) \times(0,0)$. According to (5.3), we can then write

$$
(P-z) E_{+} v=[P, \chi]_{-} K_{b}(M(z)-\text { id }) v .
$$

For $v \in L^{2}(V)$, we set

$$
\begin{aligned}
u & =E_{+} v \\
u_{-} & =E_{-+} v, \text { and } \\
R_{-} & =\frac{i}{h}[P, \chi]_{-} K_{b} .
\end{aligned}
$$

We have solved the following problem microlocally in $\left(\Omega \backslash \text { neigh }\left(m_{1}(z)\right)\right)^{2}$ (see Figure 5):

$$
\left\{\begin{aligned}
\frac{i}{h}(P-z) u+R_{-} u_{-} & =0 \\
R_{+} u & =v
\end{aligned}\right.
$$

Thus if $\mathcal{P}^{-1}$ exists, it is necessarily given by (5.1), where $E$ and $E_{-}$have yet to be defined.

For $\epsilon>0$ let

$$
\left(\Omega \times{ }_{\epsilon} \Omega\right)_{ \pm}:=\left\{\left(\bigcup_{m \in \Omega}\left(\exp \pm t H_{p}\right) m, m\right) \cap \Omega \times \Omega:-\epsilon<t<T-2 \epsilon\right\} .
$$

We will define $L_{f}$ and $L_{b}$, the forward and backward fundamental solutions (respectively) of $i(P-z) / h$, which will be defined microlocally on $\left(\Omega \times_{\epsilon} \Omega\right)_{ \pm}$, respectively. By [SjZw1, Proposition 3.5], we can conjugate $i(P-z) / h$ to $\partial_{x_{1}}$ microlocally near 


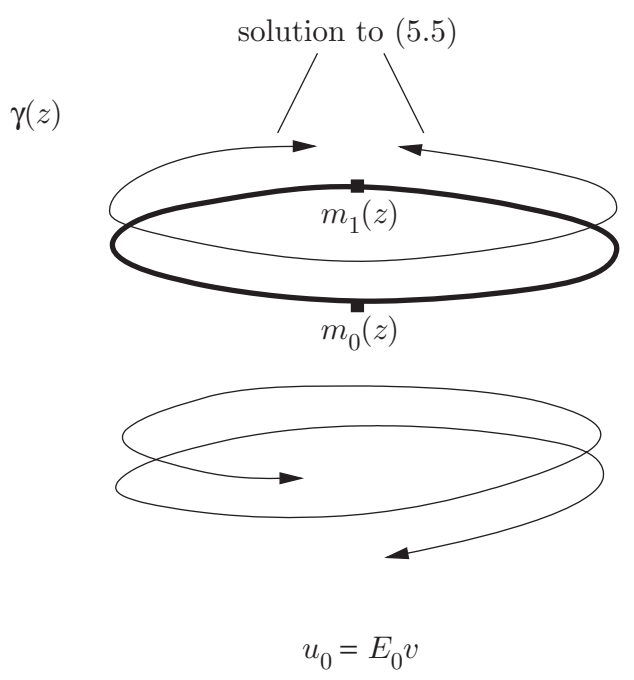

FiguRE 5. Microlocal solution to (5.5) and construction of global solution to (5.6).

the point $m_{0}(z)^{2} \in\left(T^{*} X\right)^{2}$. Then the local fundamental solutions $L_{f}^{0}$ and $L_{b}^{0}$ are given by

$$
\begin{aligned}
& L_{f}^{0} v(x)=\int_{-\infty}^{x_{1}} v\left(y, x^{\prime}\right) d y, \text { and } \\
& L_{b}^{0} v(x)=-\int_{x_{1}}^{\infty} v\left(y, x^{\prime}\right) d y,
\end{aligned}
$$

while $L_{f}$ and $L_{b}$ are now given microlocally near $\exp \left( \pm t H_{p}\right) m_{0}(z) \times m_{0}(z)$, respectively, by

$$
\begin{aligned}
L_{f} & =I_{+}^{z}(t) L_{f}^{0} \text { and } \\
L_{b} & =I_{-}^{z}(t) L_{b}^{0} .
\end{aligned}
$$

It is convenient to introduce two new microlocal cutoffs $\chi_{f}$ and $\chi_{b}$ satisfying (4.3)(4.4) and, in addition,

$$
\begin{aligned}
& \chi \equiv 1 \text { on } \operatorname{supp} \chi_{f} \cap W_{+}, \\
& \chi_{b} \equiv 1 \text { on } \operatorname{supp} \chi \cap W_{+},
\end{aligned}
$$

where $W_{+}$is a neighbourhood of $m_{0}(z)$ containing the support of $[P, \chi]_{+}$(see Figure 6). For $v \in L^{2}(\Omega)$, set

$$
\tilde{u}=L_{f}(I-\chi) v,
$$

and observe that $(P-z) \tilde{u}=0$ past the support of $(I-\chi)$ in the direction of the $H_{p}$ flow. In particular, $(P-z) \tilde{u}=0$ on $\operatorname{supp} \chi_{f}$. Then past $\operatorname{supp}(I-\chi)$ in the direction of the $H_{p}$ flow, we have according to Remark 4.2 .

$$
\begin{aligned}
\tilde{u} & =K K^{*} \frac{i}{h}\left[P, \chi_{f}\right]_{+} \tilde{u} \\
& =K K^{*} \frac{i}{h}\left[P, \chi_{f}\right]_{+} L_{f}(I-\chi) v .
\end{aligned}
$$




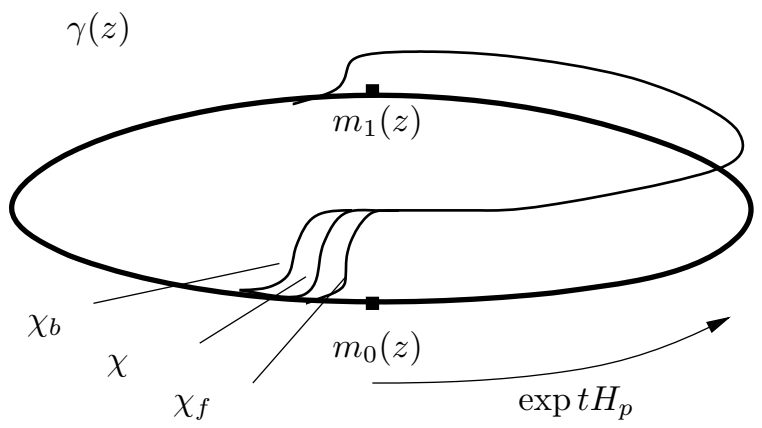

Figure 6 . The cutoffs $\chi_{b}, \chi$, and $\chi_{f}$.

Let $\widetilde{I}_{+}(t)$ be the extension of $I_{+}(t)$ to $T \leq t \leq 2 T-\epsilon$, and let $\widetilde{K}_{f}=\widetilde{I}_{+} K$. Let $\widetilde{\Omega}$ denote the double covering space of $\Omega$. Then in $\widetilde{\Omega}$,

$$
\tilde{u}=\widetilde{K}_{f} K^{*} \frac{i}{h}\left[P, \chi_{f}\right]_{+} L_{f}(I-\chi) v .
$$

That is, on the first pass around $\gamma(z)$,

$$
\tilde{u}=L_{f}(I-\chi) v
$$

while on the second pass, we just keep propagating in the forward direction, and (writing id $=K K^{*} \frac{i}{h}\left[P, \chi_{f}\right]_{+}$as usual), replace $K$ with $\widetilde{K}_{f}$ when we have propagated past $m_{0}(z)$ again. We define $\hat{u}=L_{b} \chi v$ and $\widetilde{K}_{b}=\widetilde{I}_{-} K$ similar to $\widetilde{K}_{f}$ so that

$$
\hat{u}=\widetilde{K}_{b} K^{*} \frac{i}{h}\left[P, \chi_{b}\right]_{+} L_{b} \chi v .
$$

We think of $\tilde{u}$ and $\hat{u}$ as being double-valued on $\Omega$ and write $L_{f f} v$ and $L_{b b} v$ to denote the second branches, respectively, in a neighbourhood of $m_{1}(z)$. Let $W_{-}$denote a neighbourhood of $m_{1}(z)$, and define (see Figure 5)

$$
u_{0}=E_{0} v:=\left\{\begin{array}{l}
L_{b} \chi v+L_{f}(I-\chi) v \text { outside } W_{-}, \\
L_{b} \chi v+(I-\chi) L_{b b} \chi v+L_{f}(I-\chi) v+\chi L_{f f}(I-\chi) v \text { in } W_{-}
\end{array} .\right.
$$

Now we apply $i(P-z) / h$ to $E_{0} v$ in $W_{-}$:

$$
\begin{aligned}
\frac{i}{h}(P-z) E_{0} v= & v-\frac{i}{h}[P, \chi]_{-} L_{b b} \chi v+\frac{i}{h}[P, \chi]_{-} L_{f f}(I-\chi) v \\
= & v-\frac{i}{h}[P, \chi]_{-} K_{b} K^{*} \frac{i}{h}[P, \chi]_{+} L_{b} \chi v \\
& \quad+\frac{i}{h}[P, \chi]_{-} K_{f} K^{*} \frac{i}{h}[P, \chi]_{+} L_{f}(I-\chi) v \\
= & v-\frac{i}{h}[P, \chi]_{-} K_{b}\left(K^{*} \frac{i}{h}[P, \chi]_{+} L_{b} \chi v\right. \\
& \left.-M(z) K^{*} \frac{i}{h}[P, \chi]_{+} L_{f}(I-\chi) v\right),
\end{aligned}
$$

where we have used $K_{f}=K_{b} M(z)$ in $W_{-}$and dropped the tilde and hat notation when thinking of second branches. We have solved the following problem:

$$
\frac{i}{h}(P-z) E_{0} v+R_{-} E_{0,-} v=v,
$$


with

$$
R_{-}=\frac{i}{h}[P, \chi]_{-} K_{b}
$$

as above, and

$$
E_{0,-} v:=K^{*} \frac{i}{h}[P, \chi]_{+} L_{b} \chi v-M(z) K^{*} \frac{i}{h}[P, \chi]_{+}(I-\chi) v .
$$

Recalling the structure of $\mathcal{E}$ and $\mathcal{P}$, we calculate

$$
\mathcal{P} \mathcal{E}=\left(\begin{array}{ll}
\frac{i}{h}(P-z) E+R_{-} E_{-} & \frac{i}{h}(P-z) E_{+}+R_{-} E_{-+} \\
R_{+} E & R_{+} E_{+}
\end{array}\right),
$$

so that if $\mathcal{E}$ is to be a microlocal right inverse of $\mathcal{P}$ near $\gamma \times(0,0)$, we require

$$
\begin{aligned}
\frac{i}{h}(P-z) E+R_{-} E_{-} & =\mathrm{id}: L^{2}(\Omega) \rightarrow L^{2}(\Omega), \\
\frac{i}{h}(P-z) E_{+}+R_{-} E_{-+} & =0: L^{2}(V) \rightarrow L^{2}(\Omega), \\
R_{+} E & =0: L^{2}(\Omega) \rightarrow L^{2}(V), \text { and } \\
R_{+} E_{+} & =\mathrm{id}: L^{2}(V) \rightarrow L^{2}(V)
\end{aligned}
$$

microlocally. Note that (5.8) and (5.10) are satisfied according to (5.5). Owing to (5.10), if we write $E=\left(I-E_{+} R_{+}\right) \tilde{E}$ for some $\tilde{E}$, then

$$
R_{+} E=R_{+}\left(I-E_{+} R_{+}\right) \tilde{E}=\left(I-R_{+} E_{+}\right) R_{+} \tilde{E}=0,
$$

and comparing with (5.7) we see $\tilde{E}=E_{0}$,

$$
E=E_{0}+E_{+} R_{+} E_{0}
$$

and

$$
E_{-}=E_{0,-}-E_{-+} R_{+} E_{0} .
$$

Thus $\mathcal{E}$ is a right inverse. To see that it is also a left inverse, observe that

$$
\begin{aligned}
R_{+}^{*} & =\frac{i}{h}[P, \chi]_{+} K \text { and } \\
R_{-}^{*} & =K_{b}^{*} \frac{i}{h}[P, \chi]_{-},
\end{aligned}
$$

together with

$$
K_{b}^{*} \frac{i}{h}[P, \chi]_{-} K_{b}=-\mathrm{id}
$$

implies

$$
K_{b}^{*} \frac{i}{h}[P,(I-\chi)]_{-} K_{b}=\mathrm{id} .
$$

In other words, after exchanging $\chi$ with $1-\chi, W_{+}$with $W_{-}$, and $K$ with $K_{b}, R_{+}^{*}$ has the same form as $R_{-}$and $R_{-}^{*}$ has the same form as $R_{+}$. Thus

$$
\mathcal{P}^{*}=\left(\begin{array}{cc}
\frac{i}{h}(P-z) & R_{+}^{*} \\
R_{-}^{*} & 0
\end{array}\right)
$$

has the same form as $\mathcal{P}$ and hence has a microlocal right inverse, say

$$
\mathcal{F}^{*}:=\left(\begin{array}{cc}
F & F_{+} \\
F_{-} & F_{-+}
\end{array}\right)^{*} .
$$


Then $\mathcal{P}^{*} \mathcal{F}^{*}=$ id implies $\mathcal{F P}=$ id, so

$$
\mathcal{F}=\mathcal{F P} \mathcal{E}=\mathcal{E}
$$

implies $\mathcal{F}=\mathcal{E}$.

As every operator used in the preceding construction depends holomorphically on $z \in\left[-\epsilon_{0}, \epsilon_{0}\right]+i\left(-c_{0} h, c_{0} h\right)$, we have proved the following proposition, which is from [SjZw1:

Proposition 5.1. With $\mathcal{P}$ and $\mathcal{E}$ as above and $z \in\left[-\epsilon_{0}, \epsilon_{0}\right]+i\left(-c_{0} h, c_{0} h\right), \mathcal{E}$ is a microlocal inverse for

$$
\mathcal{P}: L^{2}(\Omega) \times L^{2}(V) \rightarrow L^{2}(\Omega) \times L^{2}(V)
$$

near $\gamma \subset T^{*} X$, and, in addition,

$$
\|\mathcal{E}\|_{L^{2}(\Omega) \times L^{2}(V) \rightarrow L^{2}(\Omega) \times L^{2}(V)} \leq C .
$$

5.3. Comparing $P-z$ to $M(z)$. As a consequence of Proposition 5.1 and motivated by the linear algebra Grushin problem, the following two theorems show quantitatively that $P-z$ is invertible if and only if $I-M(z)$ is invertible.

Theorem 4. Let $M(z): L^{2}(V) \rightarrow L^{2}(V)$ be the Quantum Monodromy operator, $(P-z)$ and $R_{+}$as above. Suppose $A \in \Psi_{h}^{0,0}\left(T^{*} X\right)$ is a microlocal cutoff with wavefront set sufficiently close to $\gamma \subset T^{*} X$ and $B \in \Psi_{h}^{0,0}\left(T^{*} V\right)$ is a microlocal cutoff with wavefront set sufficiently close to $(0,0) \in T^{*} V$. Then there exists $\epsilon_{0}>0$, $c_{0}>0$, and $h_{0}>0$ such that, with $z \in\left[-\epsilon_{0}, \epsilon_{0}\right]+i\left(-c_{0} h, c_{0} h\right)$ and $0<h<h_{0}$,

$$
\begin{gathered}
\|(P-z) u\|_{L^{2}(X)} \geq C^{-1} h\left(\left\|B(I-M(z)) R_{+} u\right\|_{L^{2}(V)}-\|(I-A) u\|_{L^{2}(X)}\right) \\
-\mathcal{O}\left(h^{\infty}\right)\|u\|_{L^{2}(X)} .
\end{gathered}
$$

Further,

$$
\begin{aligned}
\|A u\|_{L^{2}(X)} \leq C & \left(\left\|R_{+} u\right\|_{L^{2}(V)}+h^{-1}\|(P-z) u\|_{L^{2}(X)}+\|(I-A) u\|_{L^{2}(X)}\right) \\
& +\mathcal{O}\left(h^{\infty}\right)\|u\|_{L^{2}(X)} .
\end{aligned}
$$

Proof. That $\mathcal{E}$ is a microlocal left inverse for $\mathcal{P}$ means in particular that for $A$ and $B$ as in the statement of the theorem,

$$
\frac{i}{h} E_{-}(P-z)+E_{-+} R_{+}=l_{+}+\mathcal{O}\left(h^{\infty}\right)_{L^{2}(X) \rightarrow L^{2}(V)},
$$

where

$$
B l_{+} A=\mathcal{O}\left(h^{\infty}\right)_{L^{2}(X) \rightarrow L^{2}(V)} .
$$

Since (5.11) is only concerned with injectivity, we note that by replacing $E_{-}$and $E_{-+}$with $\tilde{E}_{-}=B E_{-}$and $\tilde{E}_{-+}=B E_{-+}$, respectively, in (5.13) doesn't change the fact that $\mathcal{E}$ is a microlocal left inverse. Thus

$$
\frac{i}{h} \tilde{E}_{-}(P-z)+\tilde{E}_{-+} R_{+}=\tilde{l}_{+}+\mathcal{O}\left(h^{\infty}\right)_{L^{2}(X) \rightarrow L^{2}(V)},
$$

with

$$
\tilde{l}_{+} A:=B l_{+} A=\mathcal{O}\left(h^{\infty}\right)_{L^{2}(X) \rightarrow L^{2}(V)},
$$


and for $u \in L^{2}(X)$,

$$
\tilde{E}_{-}(P-z) u+\frac{h}{i} \tilde{E}_{-+} R_{+} u=\frac{h}{i} \tilde{l}_{+}(I-A) u+\mathcal{O}\left(h^{\infty}\right)\|u\|_{L^{2}(X)},
$$

hence (5.11).

For (5.12), we note $\mathcal{E P}=$ id microlocally also gives

$$
\frac{i}{h} E(P-z)+E_{+} R_{+}=\mathrm{id}_{\left.L^{2}(X)\right) \rightarrow L^{2}(X)}+l,
$$

where

$$
A l A=\mathcal{O}\left(h^{\infty}\right)_{L^{2}(X) \rightarrow L^{2}(X)} .
$$

Similar to (5.14), we replace $E$ and $E_{+}$with $\tilde{E}=A E$ and $\tilde{E}_{+}=A E_{+}$without changing that $\mathcal{E}$ is a microlocal left inverse of $\mathcal{P}$, and from (5.15), we get for $u \in$ $L^{2}(X)$,

$$
\frac{i}{h} \tilde{E}(P-z) u+\tilde{E}_{+} R_{+} u=A u+\tilde{l} u+\mathcal{O}\left(h^{\infty}\right)\|u\|_{L^{2}(X)} .
$$

Using $\tilde{l} A u:=A l A u=\mathcal{O}\left(h^{\infty}\right) u$, we get

$$
C\left(\|(P-z) u\|_{L^{2}(X)}+h\left\|R_{+} u\right\|_{L^{2}(V)}\right) \geq h\|A u\|_{L^{2}(X)}-h\|(I-A) u\|_{L^{2}(X)},
$$

which is (5.12).

Using that $\mathcal{E}$ is a microlocal right inverse for $\mathcal{P}$ we obtain the following theorem, which completes the correspondence between $I-M(z)$ and $P-z$.

Theorem 5. Suppose $A \in \Psi^{0,0}(X), B \in \Psi^{0,0}(V)$ satisfy

$$
\begin{aligned}
& A \equiv 1 \text { microlocally near } \gamma, \\
& A \equiv 0 \text { microlocally away from } \gamma, \\
& B \equiv 1 \text { microlocally near }(0,0), \\
& B \equiv 0 \text { microlocally away from }(0,0) .
\end{aligned}
$$

Suppose $u \in L^{2}(X)$ satisfies

$$
A u=u+\mathcal{O}\left(h^{\infty}\right)\|u\|_{L^{2}(X)},
$$

and $v \in L^{2}(V)$ satisfies

$$
B v=v+\mathcal{O}\left(h^{\infty}\right)\|v\|_{L^{2}(V)} .
$$

Then we have

$$
\begin{aligned}
A \frac{i}{h}(P-z) E u+A R_{-} E_{-} u & =u+\mathcal{O}\left(h^{\infty}\right)\|u\|_{L^{2}(X)}, \text { and } \\
A \frac{i}{h}(P-z) E_{+} v+A R_{-}(I-M(z)) v & =\mathcal{O}\left(h^{\infty}\right)\|v\|_{L^{2}(V)} .
\end{aligned}
$$

Remark 5.2. The utility of (5.17) is that in $\$ 10$, where $\gamma$ will be assumed elliptic instead of semi-hyperbolic, we construct $v \in L^{2}(V)$ concentrated near $(0,0)$ satisfying

$$
(I-M(z)) v=\mathcal{O}\left(h^{N}\right), \quad \forall N .
$$

Then $u:=E_{+} v$ satisfies

$$
(P-z) u=\mathcal{O}\left(h^{N+1}\right)\|u\|_{L^{2}(X)}
$$

microlocally near $\gamma$. This provides essentially a converse to our Main Theorem. 
Proof of Theorem [5. From Proposition [5.1] if we multiply $\mathcal{P}$ by $\mathcal{E}$ on the right, we get

$$
\begin{aligned}
\frac{i}{h}(P-z) E+R_{-} E_{-} & =\operatorname{id}_{L^{2}(X) \rightarrow L^{2}(X)}+r \\
\frac{i}{h}(P-z) E_{+}+R_{-}(I-M(z)) & =r_{-} \\
R_{+} E & =r_{+} \\
R_{+} E_{+} & =\operatorname{id}_{L^{2}(V) \rightarrow L^{2}(V)}+r_{-+},
\end{aligned}
$$

where

$$
\begin{aligned}
A r A & =\mathcal{O}\left(h^{\infty}\right)_{L^{2}(X) \rightarrow L^{2}(X)}, \\
A r_{-} B & =\mathcal{O}\left(h^{\infty}\right)_{L^{2}(V) \rightarrow L^{2}(X),} \\
B r_{+} A & =\mathcal{O}\left(h^{\infty}\right)_{L^{2}(X) \rightarrow{ }^{2}(V)}, \text { and } \\
B r_{-+} B & =\mathcal{O}\left(h^{\infty}\right)_{L^{2}(V) \rightarrow L^{2}(V) .}
\end{aligned}
$$

Hence (5.18) -(5.19) imply that for any $u \in L^{2}(X), v \in L^{2}(V)$,

$$
A \frac{i}{h}(P-z) E u+A R_{-} E_{-} u=A u+A r(I-A) u+\mathcal{O}\left(h^{\infty}\right)\|u\|_{L^{2}(X)}
$$

and

$$
A \frac{i}{h}(P-z) E_{+} v+A R_{-}(I-M(z)) v=A r_{-}(I-B) v+\mathcal{O}\left(h^{\infty}\right)\|v\|_{L^{2}(V)},
$$

which is (5.16)-(5.17).

\section{THE MOdel CASE}

In this section we indicate how Theorem 4 can be used to estimate $P-z$ in the model case. Let $\operatorname{dim} X=2$, and assume $t$ parametrizes $\gamma=\gamma(0)$ and $\tau$ is the dual variable to $t$. Then our model for $p$ near $\gamma$ is the symbol

$$
p=\tau+\lambda x \xi,
$$

with $\lambda>0$. We have

$$
H_{p}=\partial_{t}+\lambda\left(x \partial_{x}-\xi \partial_{\xi}\right),
$$

and the Poincaré map $S: \mathbb{R}^{2} \rightarrow \mathbb{R}^{2}$ is given by

$$
S=\left(\begin{array}{cc}
e^{\lambda} & 0 \\
0 & e^{-\lambda}
\end{array}\right) .
$$

We want a deformation of the identity into $S$, that is, a smooth family of symplectomorphisms $\kappa_{t}$ such that $\kappa_{0}=$ id and $\kappa_{1}=S$. This is clear in the model case:

$$
\kappa_{t}=\exp t\left(\begin{array}{cc}
\lambda & 0 \\
0 & -\lambda
\end{array}\right)
$$

According to [SjZw1, Lemma 3.2] (see also [EvZw]), we can find a time-dependent effective Hamiltonian $q_{t}=q_{t}(x, \xi)$ such that

$$
\frac{d}{d t} \kappa_{t}=\left(\kappa_{t}\right)_{*} H_{q_{t}} .
$$

In the model case, this is again clear: $q_{t}=\lambda x \xi$, independent of $t$. 
We know in general that if $M(z)$ is the Quantum Monodromy operator, then it is an $h$-FIO associated to the graph of $S$, which means that our model is $M(z)=$ $M^{z}(1)$ for $M^{z}(t)$ a family of $h$-FIOs satisfying

$$
\left\{\begin{array}{l}
h D_{t} M^{z}(t)+Q(t) M^{z}(t)=0 \\
M^{z}(0)=\mathrm{id}
\end{array}\right.
$$

where $Q(t)=\mathrm{Op}\left(q_{t}\right)$ for the effective Hamiltonian $q_{t}$ as above. In the model case, $q$ does not depend on $t$ or $z$, so with $Q=\mathrm{Op}(q), M^{z}(t)$ is just the semigroup

$$
M(t)=\exp \left(-\frac{i}{h} t Q\right) .
$$

The basic idea is that $M(t)$ is unitary, but $e^{-G^{w}} M(t) e^{G^{w}}$ is not for $G$ with real principal symbol (if it exists). Further, in the model case, if $G$ is independent of $t$,

$$
e^{-G^{w}} M(t) e^{G^{w}}=\exp \left(-\frac{i}{h} t e^{-G^{w}} Q e^{G^{w}}\right),
$$

and it will suffice to show $e^{-G^{w}} Q e^{G^{w}}$ has an imaginary part of fixed size comparable to $h$.

As in Lemma 2.1 for $u \in L^{2}\left(\mathbb{R}^{n}\right)$ we define $T_{h, \tilde{h}}$ by

$$
T_{h, \tilde{h}} u(X):=(h / \tilde{h})^{\frac{n}{4}} u\left((h / \tilde{h})^{\frac{1}{2}} X\right) .
$$

We then conjugate $M(t)$ to $M_{1}(t)=T_{h, \tilde{h}} M(t) T_{h, \tilde{h}}^{-1}$ and observe $M_{1}(t)$ satisfies the evolution equation

$$
\begin{aligned}
h D_{t} M_{1}(t) & =-T_{h, \tilde{h}} Q M(t) T_{h, \tilde{h}}^{-1} \\
& =-T_{h, \tilde{h}} Q T_{h, \tilde{h}}^{-1} T_{h, \tilde{h}} M(t) T_{h, \tilde{h}}^{-1} \\
& =-Q_{1} M_{1}(t),
\end{aligned}
$$

where

$$
Q_{1}=T_{h, \tilde{h}} Q T_{h, \tilde{h}}^{-1} \in \Psi_{-\frac{1}{2}}^{-\infty, 0,0}
$$

microlocally (we refer the reader to $₫ 2$ for the definitions). We write $q_{1}(X, \Xi)=$ $\sigma_{\tilde{h}}\left(Q_{1}\right)$, where

$$
q_{1}(X, \Xi)=\lambda(h / \tilde{h}) X \Xi+\mathcal{O}\left(h^{2}+\tilde{h}^{2}\right)
$$

as in Lemma 2.1

Now we define the escape function

$$
G(X, \Xi)=\frac{1}{2} \log \left(\frac{1+X^{2}}{1+\Xi^{2}}\right)
$$

and according to a result of Bony and Chemin BoCh (see also [Chr1, Lemma 2.1]), we can form the family of operators

$$
e^{s G^{w}}
$$

where $G^{w}$ is the Weyl quantization of $G$ in the $\tilde{h}$ calculus and $|s|$ is sufficiently small. Let

$$
\widetilde{M}(t)=e^{-s G^{w}} M_{1}(t) e^{s G^{w}}
$$


whence

$$
h D_{t} \widetilde{M}(t)=-\widetilde{Q} \widetilde{M}(t)
$$

for

$$
\widetilde{Q}=e^{-s G^{w}} Q_{1} e^{s G^{w}}
$$

by a similar argument to (6.2)-6.4). We write

$$
\widetilde{Q}=\exp \left(-s \operatorname{ad}_{G^{w}}\right) Q_{1},
$$

with

$$
\operatorname{ad}_{G^{w}}^{k} Q_{1}=\mathcal{O}_{L^{2} \rightarrow L^{2}}\left(h \tilde{h}^{k-1}\right)
$$

and

$$
\left[Q_{1}, G^{w}\right]=-i \tilde{h} \mathrm{Op}_{\tilde{h}}^{w}\left(H_{q_{1}} G\right)+\mathcal{O}\left(h^{3 / 2} \tilde{h}^{3 / 2}\right)
$$

We have

$$
\begin{aligned}
H_{q_{1}} G & =\lambda(h / \tilde{h})\left(\frac{X^{2}}{1+X^{2}}+\frac{\Xi^{2}}{1+\Xi^{2}}\right) \\
& =: \lambda(h / \tilde{h}) A,
\end{aligned}
$$

so that

$$
\widetilde{Q}=Q_{1}-i s h \mathrm{Op}_{\tilde{h}}^{w}(A)+s E_{1}^{w}+s^{2} E_{2}^{w},
$$

with $E_{1}=\mathcal{O}\left(h^{3 / 2} \tilde{h}^{3 / 2}\right)$ and $E_{2}=\mathcal{O}(h \tilde{h})$. Since $A$ is roughly the harmonic oscillator (see [Chr1, Lemma 5.1]),

$$
\left\langle\mathrm{Op}_{\tilde{h}}^{w}(A) U, U\right\rangle \geq \frac{\tilde{h}}{C}\|U\|^{2}
$$

independently of $h$, so that

$$
\operatorname{Im}\langle\widetilde{Q} U, U\rangle \leq-\frac{h \tilde{h}}{C}\|U\|^{2} .
$$

Thus with $\tilde{h}>0$ small but fixed,

$$
\widetilde{M}(1)=\exp \left(-\frac{i}{h}(\operatorname{Re} \widetilde{Q}+i \operatorname{Im} \widetilde{Q})\right)
$$

and by (6.5),

$$
\|\widetilde{M}(1)\|_{L^{2}(\mathbb{R}) \rightarrow L^{2}(\mathbb{R})} \leq r<1 .
$$

For $u \in L^{2}(\mathbb{R})$ and $U=T_{h, \tilde{h}} u$, we have by (6.5) and (6.6),

$$
\operatorname{Re}\langle(I-\widetilde{M}(1)) U, U\rangle \geq C^{-1}\|U\|^{2}
$$

for some $0<C<\infty$. Define the operator $K^{w}$ by

$$
e^{s K^{w}}=T_{h, \tilde{h}}^{-1} e^{s G^{w}} T_{h, \tilde{h}} .
$$

We have shown that

$$
\operatorname{Re}\left\langle e^{-s K^{w}}(I-M) e^{s K^{w}} u, u\right\rangle \geq C^{-1}\|u\|^{2} .
$$


Since $\left\|\exp \left( \pm s K^{w}\right)\right\|_{L^{2} \rightarrow L^{2}}=\mathcal{O}\left(h^{-N}\right)$ for some $N$, we have

$$
\operatorname{Re}\langle(I-M) u, u\rangle \geq C h^{N}\|u\|^{2} .
$$

With this estimate on $(I-M)$, we now apply Theorem 4 to conclude the microlocal invertibility of $P-z$ in this model case.

\section{The LinEARIZATION}

7.1. Symplectic linear algebra and matrix logarithms. In this section, we will show how to reduce the case of a general Poincaré map with a fixed point to studying the quadratic Birkhoff normal forms. We assume as in the introduction that the eigenvalues of modulus 1 obey the nonresonance assumption (1.2).

We begin by tackling the problem of negative real eigenvalues and eigenvalues of modulus 1 of the linearized Poincaré map. Let $S: W_{1} \rightarrow W_{2}, W_{1}, W_{2} \subset \mathbb{R}^{2 n-2}$, be a local symplectic map, $S(0,0)=(0,0)$, which we have identified with its coordinate representation. We consider the polar decomposition of $d S(0,0)$ :

$$
d S(0,0)=\exp (-J F) \exp (B),
$$

with $F$ and $B$ real-valued and $\exp (B)$ positive definite and symplectic. Specifically, $\exp (-J F)$ describes the action due to the eigenvalues of modulus 1 as well as the rotation inherent in the negative real eigenvalues. We consider first $A=\exp (B)$. We denote by $\left\{\mu_{j}\right\}$ the eigenvalues of $A$ and by $\left\{\tilde{\mu}_{j}\right\}$ the eigenvalues of $d S(0,0)$. Let $\mu$ be an eigenvalue of $A$. Then $A$ symplectic implies that if $\mu>1$ is real, then $\mu^{-1}$ is also an eigenvalue, and if $\mu$ is complex, $|\mu|>1$, then $\mu^{-1}, \bar{\mu}$, and $\bar{\mu}^{-1}$ are also eigenvalues. If $\tilde{\mu},|\tilde{\mu}|=1$ is an eigenvalue of $d S(0,0)$, then $\tilde{\tilde{\mu}}=\mu^{-1}$ is also an eigenvalue $d S(0,0)$, but we will see that neither of these contributes to $A$. If $\tilde{E}_{\mu}$ is the generalized complex eigenspace of $\mu$, then we can put $A$ into complex Jordan form over $\tilde{E}_{\mu}$. To keep the change of variables symplectic, we observe that $\tilde{E}_{\mu^{-1}}$ is the dual eigenspace to $\tilde{E}_{\mu}$, so if

$$
A_{\mu}:=\left.A\right|_{\tilde{E}_{\mu}}=\left(\begin{array}{ccccc}
\mu & 1 & 0 & \ldots \ldots \\
0 & \mu & 1 & 0 & \ldots \\
\vdots & \ddots & \ddots & \ldots \ldots \\
0 & \ldots & 0 & \mu & 1 \\
0 & \ldots & \ldots & \ldots & \mu
\end{array}\right)
$$

then symplectically completing this basis in $\tilde{E}_{\mu} \oplus \tilde{E}_{\mu^{-1}}$ gives

$$
\left.A\right|_{\tilde{E}_{\mu-1}}=\left(A_{\mu}^{T}\right)^{-1} \text {. }
$$

As $A_{\mu}=\mu I+N_{\mu}$ with $N_{\mu}$ nilpotent, by expanding $\left(A_{\mu}^{T}\right)^{-1}$ as a power series, we see

$$
A_{\mu^{-1}}:=\left.A\right|_{\tilde{E}_{\mu^{-1}}}=\mu^{-1} I+N_{\mu^{-1}}
$$

with $N_{\mu^{-1}}$ nilpotent. We choose a branch of the logarithm so that

$$
\lambda(\mu)=\log (\mu)
$$

satisfies

$$
\begin{aligned}
\lambda\left(\mu^{-1}\right) & =-\lambda(\mu) \text { and } \\
\lambda(\bar{\mu}) & =\overline{\lambda(\mu)}
\end{aligned}
$$


and observe for $N$ nilpotent,

$$
\log (I+N)=N-\frac{N^{2}}{2}+\frac{N^{3}}{3}+\ldots
$$

is a finite series. Then we can define

$$
\log \left(\mu I+N_{\mu}\right)=\lambda(\mu)+N_{\lambda}
$$

with $N_{\lambda}$ nilpotent.

We apply this technique to each generalized eigenspace of $A$ to obtain a complex matrix

$$
\widetilde{B}:=\log A .
$$

We see $\widetilde{B}$ is block diagonal with diagonal elements of the form $\lambda I+N_{\lambda}$ with $N_{\lambda}$ nilpotent. We know $|\mu|>1$ real gives $\operatorname{Re} \lambda(\mu)>0$. For $\lambda$ satisfying $\operatorname{Re} \lambda>0$, let $E_{\lambda}$ denote the generalized complex eigenspace of $\lambda$ with respect to $\widetilde{B}$, and let $\tilde{E}_{\tilde{\mu}}$ denote the generalized complex eigenspace of $\tilde{\mu}$ with respect to $d S(0,0)$. There are 4 cases to consider.

Case 1: $\tilde{\mu}>1$ is real and an eigenvalue of $d S(0,0)$. Then $E_{\lambda} \oplus E_{-\lambda}$ is a real symplectic space which is equal to $\tilde{E}_{\tilde{\mu}} \oplus \tilde{E}_{\tilde{\mu}^{-1}}$. If we put $\widetilde{B}$ into Jordan form over $E_{\lambda}$

$$
\widetilde{B}_{\lambda}:=\left.\widetilde{B}\right|_{E_{\lambda}}=\left(\begin{array}{ccccc}
\lambda & 1 & 0 & \ldots \ldots \\
0 & \lambda & 1 & 0 & \ldots \\
\vdots & \ddots & \ddots & \ldots \ldots \\
0 & \ldots & 0 & \lambda & 1 \\
0 & \ldots & \ldots & \ldots & \lambda
\end{array}\right)
$$

completing the basis symplectically over $E_{\lambda} \oplus E_{-\lambda}$ gives

$$
\left.\widetilde{B}\right|_{E_{-\lambda}}=-\left(\widetilde{B}_{\lambda}\right)^{T}
$$

As $\mu=\tilde{\mu}$ was an eigenvalue of $A$,

$$
\left.\exp (-J F)\right|_{\tilde{E}_{\tilde{\mu}} \oplus \tilde{E}_{\tilde{\mu}-1}}=\mathrm{id} .
$$

Case 2: $\tilde{\mu}$ is complex, $|\tilde{\mu}|>1$, and $\tilde{\mu}$ is an eigenvalue of $d S(0,0)$. Then $E_{\lambda} \oplus E_{-\lambda} \oplus$ $E_{\bar{\lambda}} \oplus E_{-\bar{\lambda}}$ is the complexification of a real symplectic vector space which is equal to $\tilde{E}_{\tilde{\mu}} \oplus \tilde{E}_{\tilde{\mu}^{-1}} \oplus \tilde{E}_{\bar{\mu}} \oplus \tilde{E}_{\tilde{\tilde{\mu}}^{-1}}$. Changing variables as in [Chr1, $\left.\S 6\right]$, we see

$$
\left.\widetilde{B}\right|_{E_{\lambda} \oplus E_{-\lambda} \oplus E_{\bar{\lambda}} \oplus E_{-\bar{\lambda}}}=\left(\begin{array}{cc}
\widetilde{B}_{\lambda} & 0 \\
0 & -\left(\widetilde{B}_{\lambda}\right)^{T}
\end{array}\right),
$$

where

$$
\widetilde{B}_{\lambda}=\left(\begin{array}{ccccc}
\Lambda & I & 0 & \ldots \ldots \\
0 & \Lambda & I & 0 & \ldots \\
\vdots & \ddots & \ddots & \ldots \ldots \\
0 & \ldots & 0 & \Lambda & I \\
0 & \ldots & \ldots & \Lambda & \Lambda
\end{array}\right)
$$


with $I$ the $2 \times 2$ identity matrix and

$$
\Lambda=\left(\begin{array}{cc}
\operatorname{Re} \lambda & -\operatorname{Im} \lambda \\
\operatorname{Im} \lambda & \operatorname{Re} \lambda
\end{array}\right)
$$

Further,

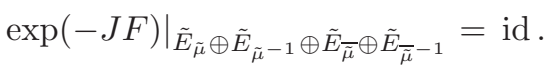

Case 3: $\mu>1$ is real, and $\tilde{\mu}=-\mu$ is an eigenvalue of $d S(0,0) . E_{\lambda} \oplus E_{-\lambda}$ is a real symplectic vector space, equal to $\tilde{E}_{\tilde{\mu}} \oplus \tilde{E}_{\tilde{\mu}^{-1}}$ and $\widetilde{B}$ is handled as in Case 1, with the important difference:

$$
\left.\exp (-J F)\right|_{\tilde{E}_{\tilde{\mu}} \oplus \tilde{E}_{\tilde{\mu}-1}}=-\mathrm{id} .
$$

Case 4: $|\tilde{\mu}|=1, \operatorname{Im} \tilde{\mu}>0$ is an eigenvalue of $d S(0,0)$. Then $E_{\lambda} \oplus E_{-\lambda}$ is a complex symplectic vector space which is the complexification of a real symplectic vector space which is equal to $\tilde{E}_{\tilde{\mu}} \oplus \tilde{E}_{\tilde{\mu}^{-1}}$. Since we have assumed in particular that $\tilde{\mu}$ occurs with multiplicity 1 , so does $\lambda$. Write $\lambda=i \alpha, \alpha>0$, in which case we observe that

$$
\left.F\right|_{E_{i \alpha} \oplus E_{-i \alpha}}=\left(\begin{array}{cc}
\alpha & 0 \\
0 & \alpha
\end{array}\right)
$$

is diagonal since $\tilde{\mu}$ is distinct.

We have proved the following proposition, which we record in detail to fix our notation.

Proposition 7.1. Let $S: W_{1} \rightarrow W_{2}, W_{1}, W_{2} \subset \mathbb{R}^{2 n-2}$ be a local symplectic map, $S(0,0)=(0,0)$, and let $n_{h c}$ be the number of Jordan blocks of complex eigenvalues $\mu$ of $d S(0,0)$ satisfying $|\mu|>1, \operatorname{Re} \mu>1$, and $\operatorname{Im} \mu>0$. Let $n_{h r+}$ be the number of Jordan blocks of real positive eigenvalues $\mu$ of $d S(0,0)$ satisfying $\mu>1, n_{h r-}$ be the number of Jordan blocks of negative real eigenvalues $-\mu$ of $d S(0,0)$ satisfying $-\mu<-1$, and $n_{e}$ be the number of eigenvalues $\mu$ of modulus 1 satisfying $\operatorname{Im} \mu>0$. For

$$
\begin{aligned}
j \in\left(1, \ldots, n_{h c} ; 2 n_{h c}+1, \ldots, 2 n_{h c}+n_{h r+} ;\right. \\
2 n_{h c}+n_{h r+}+1, \ldots, 2 n_{h c}+n_{h r+}+n_{h r-} ; \\
\left.2 n_{h c}+n_{h r+}+n_{h r-}+1, \ldots, 2 n_{h c}+n_{h r+}+n_{h r-}+n_{e}\right)
\end{aligned}
$$

let $k_{j}$ denote the multiplicity of $\mu_{j}$ so that

$$
\begin{aligned}
2 n-2=4\left(\sum_{j=1}^{n_{h c}} k_{j}\right) & +2\left(\sum_{j=2 n_{h c}+1}^{2 n_{h c}+n_{h r+}} k_{j}\right)+2\left(\sum_{2 n_{h c}+n_{h r+}+1}^{2 n_{h c}+n_{h r+}+n_{h r-}} k_{j}\right) \\
& +2\left(\sum_{2 n_{h c}+n_{h r+}+n_{h r-}+1}^{2 n_{h c}+n_{h r+}+n_{h r-}+n_{e}} k_{j}\right) .
\end{aligned}
$$

Choose $\lambda_{j}\left(\mu_{j}\right)=\log \mu_{j}$ satisfying (7.1) (7.2) for $j$ in the range (7.3) and (7.5), and for $j$ in the range (7.4), choose $\lambda_{j}\left(\mu_{j}\right)=\log \left(-\mu_{j}\right)$ satisfying (7.1)-(7.2). Then there are real matrices $B$ and $F$ satisfying ${ }^{\omega} B=-B, F^{*}=F$, and a symplectic choice of coordinates such that

$$
d S(0,0)=\exp (-J F) \exp (B),
$$


and $B$ is of the form

$$
B=\operatorname{diag}\left(B_{j} ;-B_{j}^{T}\right),
$$

for $j$ in the range (7.3) (7.5). For $j \in\left(1, \ldots, n_{h c}\right), B_{j}$ is the $2 k_{j} \times 2 k_{j}$ matrix

$$
B_{j}=\left(\begin{array}{ccccc}
\Lambda_{j} & I & 0 & \ldots \ldots \\
0 & \Lambda_{j} & I & 0 & \ldots \\
\vdots & \ddots & \ddots & \ldots \ldots \\
0 & \ldots & 0 & \Lambda_{j} & I \\
0 & \ldots \ldots & \ldots & \Lambda_{j}
\end{array}\right),
$$

with $I$ the $2 \times 2$ identity matrix and

$$
\Lambda_{j}=\left(\begin{array}{cc}
\operatorname{Re} \lambda_{j} & -\operatorname{Im} \lambda_{j} \\
\operatorname{Im} \lambda_{j} & \operatorname{Re} \lambda_{j}
\end{array}\right)
$$

For $j \in\left(2 n_{h c}+1, \ldots, 2 n_{h c}+n_{h r+}+n_{h r-}\right), B_{j}$ is the $k_{j} \times k_{j}$ matrix

$$
B_{j}=\left(\begin{array}{ccccc}
\lambda_{j} & 1 & 0 & \ldots \ldots \\
0 & \lambda_{j} & 1 & 0 & \ldots \\
\vdots & \ddots & \ddots & \ldots \ldots \\
0 & \ldots & 0 & \lambda_{j} & 1 \\
0 & \ldots & \ldots & \ldots & \lambda_{j}
\end{array}\right),
$$

and for $j \in\left(2 n_{h c}+n_{h r+}+n_{h r-}+1, \ldots, 2 n_{h c}+n_{h r+}+n_{h r-}+n_{e}\right), B_{j}$ is the $1 \times 1$ matrix 0. Here

$$
F=\operatorname{diag}\left(F_{j} ; F_{j}\right),
$$

for $j$ in the range (7.3) -(7.5), where for $j \in\left(1, \ldots, 2 n_{h c}\right), F$ is the $2 k_{j} \times 2 k_{j}$ zero matrix, for $j \in\left(2 n_{h c}+1, \ldots, 2 n_{h c}+n_{h r+}\right), F_{j}$ is the $k_{j} \times k_{j}$ zero matrix, for $j \in\left(2 n_{h c}+n_{h r+}+1, \ldots, 2 n_{h c}+n_{h r+}+n_{h r-}\right)$,

$$
F_{j}=\pi I
$$

where I is the $k_{j} \times k_{j}$ identity matrix, and for $j \in\left(2 n_{h c}+n_{h r+}+n_{h r-}+1, \ldots, 2 n_{h c}+\right.$ $\left.n_{h r+}+n_{h r-}+n_{e}\right), F_{j}=\operatorname{Im} \lambda_{j}$.

We set

$$
K_{t}^{1}=\exp (-t J F) \text { and } K_{t}=\exp (t B),
$$

which we observe is the same as

$$
K_{t}^{1}=\exp \left(t H_{q^{1}}\right) \text { and } K_{t}=\exp \left(t H_{q}\right)
$$

for

$$
\begin{aligned}
q(x, \xi)= & \sum_{j=1}^{n_{h c}} \sum_{l=1}^{k_{j}}\left(\operatorname{Re} \lambda_{j}\left(x_{2 l-1} \xi_{2 l-1}+x_{2 l} \xi_{2 l}\right)-\operatorname{Im} \lambda_{j}\left(x_{2 l-1} \xi_{2 l}-x_{2 l} \xi_{2 l-1}\right)\right) \\
& +\sum_{j=1}^{n_{h c}} \sum_{l=1}^{k_{j}-1}\left(x_{2 l+1} \xi_{2 l-1}+x_{2 l+2} \xi_{2 l}\right) \\
& +\sum_{j=2 n_{h c}+1}^{2 n_{h c}+n_{h r+}+n_{h r-}}\left(\sum_{l=1}^{k_{j}} \lambda_{j} x_{l} \xi_{l}+\sum_{l=1}^{k_{j}-1} x_{l+1} \xi_{l}\right)
\end{aligned}
$$


and

$$
\text { (7.11) } q^{1}(x, \xi)=\sum_{j=2 n_{h c}+n_{h r+}+1}^{2 n_{h c}+n_{h r+}+n_{h r-}} \frac{\pi}{2}\left(x_{j}^{2}+\xi_{j}^{2}\right)+\sum_{j=2 n_{h c}+n_{h r+}+n_{h r-}+1}^{2 n_{h c}+n_{h r+}+n_{h r-}+n_{e}} \frac{\operatorname{Im} \lambda_{j}}{2}\left(x_{j}^{2}+\xi_{j}^{2}\right) \text {. }
$$

7.2. Geometry of the Poincaré section. The previous section motivates the next proposition. First we need the following lemma, which follows from the more general [Chr1, Lemma 4.2]. Recall under the assumption that $S$ is hyperbolic, the stable and unstable manifolds $\Lambda_{\mp} \subset N$ for $S$ are $(n-1)$-dimensional locally embedded transversal Lagrangian submanifolds (see [HaKa, Theorem 6.2.3]).

Lemma 7.2. Let $S: W_{1} \rightarrow W_{2}, W_{1}, W_{2} \subset \mathbb{R}^{2 n-2}, S(0,0)=(0,0)$, be a local hyperbolic symplectic map with unstable/stable manifolds $\Lambda_{ \pm}$. Then there exists a local symplectic coordinate system $(x, \xi)$ near $\gamma$ such that $\Lambda_{+}=\{\xi=0\}$ and $\Lambda_{-}=\{x=0\}$.

For the following proposition, we assume that there are no negative real eigenvalues and no eigenvalues of modulus 1 to the linearized Poincaré map. Later we will modify the general Poincaré map to be of this form. This follows from the proof of [Chr1, Proposition 4.3].

Proposition 7.3. Let $S: W_{1} \rightarrow W_{2}, W_{1}, W_{2} \subset \mathbb{R}^{2 n-2}$, be a local hyperbolic symplectic map, $S(0,0)=(0,0)$, and assume $d S(0,0)$ has no negative real eigenvalues. There is a smooth family of local symplectomorphisms $\kappa_{t}$, a smooth, real-valued matrix function $B_{t}(x, \xi)$, and a symplectic choice of coordinates in which

$$
\text { (i) } \kappa_{0}=\mathrm{id}, \kappa_{1}(x, \xi)=S(x, \xi) \text {, }
$$$$
\text { (ii) } \frac{d}{d t} \kappa_{t}=\left(\kappa_{t}\right)_{*} H_{q_{t}} \text {, }
$$

where

$$
q_{t}(x, \xi)=\left\langle B_{t}(x, \xi) x, \xi\right\rangle .
$$

Here

$$
\left\langle B_{t}(0,0) x, \xi\right\rangle=q(x, \xi),
$$

for $q(x, \xi)$ of the form (7.8) (7.10).

\section{The Proof of Theorem 1}

8.1. Motivation. We recall from Theorem 4 that if $u \in L^{2}(X)$ has wavefront set sufficiently close to $\gamma$ and $B \in \Psi^{0,0}(V)$ is a microlocal cutoff near $(0,0)$, then we have for $z \in\left[-\epsilon_{0}, \epsilon_{0}\right]+i\left(-c_{0} h, c_{0} h\right)$,

$$
\|(P-z) u\|_{L^{2}(X)} \geq C^{-1} h\left\|B(I-M(z)) R_{+} u\right\|_{L^{2}(V)} .
$$

Hence we want to show $M(z)$ has spectrum away from 1 . This is the content of the following theorem, which we state in its general form for reference.

Theorem 6. Let $\widetilde{V} \subset \mathbb{R}^{2 m}$ be an open neighbourhood of $(0,0)$, and assume $\kappa_{z}$ : $\operatorname{neigh}(\widetilde{V}) \rightarrow \kappa_{z}(\operatorname{neigh}(\widetilde{V})), \kappa_{z}(0,0)=(0,0), z \in(-\delta, \delta), \delta>0$ is a smooth family of symplectomorphisms such that $d \kappa_{z}(0,0)$ is semi-hyperbolic and the nonresonance condition (1.2) holds for $d \kappa_{z}(0,0)$. Let $M(z)$ be the microlocally unitary h-FIO which quantizes $\kappa_{z}$ as in [SjZw1, §3]. Then for $z \in\left(-\delta^{\prime}, \delta^{\prime}\right), \delta^{\prime}>0$ sufficiently small and $s \in \mathbb{R}$ sufficiently close to 0 , there exist selfadjoint, semiclassically tempered 
operators $\exp \left( \pm s K^{w}\right)$ and $R>1$ so that for $v \in L^{2}\left(\mathbb{R}^{m}\right)$ with h-wavefront set sufficiently close to $(0,0)$,

$$
\left\|e^{-s K^{w}} M(z) e^{s K^{w}} v\right\|_{L^{2}} \leq \frac{1}{R}\|v\|_{L^{2}} .
$$

From 4 we know $M(z)$ is an $h$-FIO associated to the graph of $S(z)$, where $S(z)$ is the Poincaré map for $\gamma_{z}$, the periodic orbit in the energy level $z$. Suppose for the moment that $S(z)$ satisfies the hypotheses of Proposition 7.3. and let $q_{z, t}$ be $q_{t}$ as in the conclusion of the proposition, where now $q_{z, t}$ varies over energy levels $z$ near 0 . Setting $Q_{z, t}=\mathrm{Op}_{h}^{w}\left(q_{z, t}\right)$, by modifying the proofs of the Egorov theorem and its converse [SjZw1, §3], there exists $M_{z, 0} \in \Psi_{h}^{0,0}$ microlocally unitary so that $M(z)=M^{z}(1)$ for $M^{z}(t)$ a family of operators satisfying the evolution equations

$$
\begin{aligned}
h D_{t} M^{z}+M^{z} Q_{z, t} & =0,0 \leq t \leq 1, \\
M^{z}(0) & =M_{z, 0} .
\end{aligned}
$$

In order to prove Theorem [6 we observe that if $W(z): L^{2}(V) \rightarrow L^{2}(V)$ is the microlocal inverse for $M(z)$, then we also have $W_{z, 0} \in \Psi_{h}^{0,0}$ microlocally unitary so that $W(z)=W^{z}(1)$ for $W^{z}(t)$ satisfying the following evolution equation:

$$
\begin{aligned}
h D_{t} W^{z}-Q_{z, t} W^{z} & =0,0 \leq t \leq 1, \\
W^{z}(0) & =W_{z, 0} .
\end{aligned}
$$

The rest of this section is devoted to proving that there exist semiclassically tempered operators $\exp \left( \pm s K^{w}\right)$ as in the statement of the theorem so that

$$
\left\|e^{-s K^{w}} W(z) e^{s K^{w}} v\right\|_{L^{2}(V)} \geq R\|v\|_{L^{2}(V)}
$$

for some $R>1$. Then

$$
\begin{aligned}
\|v\|_{L^{2}(V)} & =\left\|e^{-s K^{w}} W(z) e^{s K^{w}} e^{-s K^{w}} M(z) e^{s K^{w}} v\right\|_{L^{2}(V)} \\
& \geq R\left\|e^{-s K^{w}} M(z) e^{s K^{w}} v\right\|_{L^{2}(V)},
\end{aligned}
$$

which gives the theorem once we prove (8.4).

In order to get Theorem 1 from Theorem 6, we observe by (8.1) that we also have

$$
\left\|\left(I-e^{-s K^{w}} M(z) e^{s K^{w}}\right) v\right\| \geq C^{-1}\|v\| .
$$

Thus

$$
\begin{aligned}
\operatorname{Re}\left\langle e^{-s K^{w}}(I-M) e^{s K^{w}} v, v\right\rangle & =\|v\|^{2}-\operatorname{Re}\left\langle e^{-s K^{w}} M(z) e^{s K^{w}} v, v\right\rangle \\
& \geq C^{-1}\|v\|_{L^{2}(V)}^{2} .
\end{aligned}
$$

Since $\left\|\exp \left( \pm s K^{w}\right)\right\|_{L^{2} \rightarrow L^{2}}=\mathcal{O}\left(h^{-N}\right)$ for some $N$, we have

$$
\operatorname{Re}\langle(I-M(z)) v, v\rangle \geq C h^{N}\|v\|^{2} .
$$

Now let $u \in L^{2}(X)$ have wavefront set close to $\gamma$. Set $v=R_{+} u$ so that $\mathrm{WF}_{h} v$ is close to $(0,0)$, and observe with $B$ as in Theorem 4 and $b=\sigma_{h}(B)$ that $1-b$ has support away from $(0,0) \in T^{*} \mathbb{R}^{n-1}$. Then

$$
\mathrm{Op}_{h}^{w}(1-b) M(z) v=M(z) \mathrm{Op}_{h}^{w}\left(S(z)^{*}(1-b)\right) v=\mathcal{O}\left(h^{\infty}\right),
$$


so that if $\mathrm{WF}_{h} u$ is sufficiently small, then

$$
B(I-M(z)) R_{+} u=(I-M(z)) R_{+} u
$$

microlocally, and (5.12) gives the theorem.

Our biggest tool so far is the normal form deformation in Proposition 7.3. However we cannot immediately apply it to $S(z)$ satisfying the assumptions of the introduction. To get by this we will transform $S(z)$ into a hyperbolic map satisfying the assumptions of Proposition 7.3 and then later deal with the errors which come up when transforming back.

The proof of Theorem [6 will proceed in 4 basic steps. First, we deform the effective Hamiltonian into a sum of two Hamiltonians with disjoint support in $t$, one hyperbolic and one elliptic. The summed Hamiltonian will be called $q_{z, t}$. We then modify the evolution equation defining $W^{z}$ to an equation involving a conjugated version of $W^{z}, \widetilde{W}(t)$. This evolution equation will be given in terms of a conjugated quantization of $q_{z, t}, \widetilde{Q}_{z, t}$, that we will then need to estimate from below. This step is a variation on the classical idea of a "positive commutator". That is, $\mathrm{Op}_{h}^{w}\left(q_{z, t}\right)$ is selfadjoint, but if we conjugate it with an operator of the form $e^{G^{w}}$, we get $\mathrm{Op}_{h}^{w}\left(q_{z, t}\right)$ plus a lower order skew-adjoint commutator. The principal symbol of the commutator $\left[G, \mathrm{Op}\left(q_{z, t}\right)\right]$ is $h i H_{q_{z, t}} G$. The linear part of $H_{q_{z, t}}$ is block diagonal in the hyperbolic and elliptic variables, but the nonlinear part potentially forces interaction between the hyperbolic and elliptic variables. Hence we will be forced to introduce a complex weight $G$ to gain some orthogonality between the hyperbolic and elliptic variables. This is accomplished in Step 3. Finally we will estimate $M$, the inverse of which is related to $\widetilde{W}$ by conjugation.

8.2. Step 1: Deform $q_{z, t}$. We construct a rescaled deformation of the identity into $S(z)$ in which the elliptic part of the effective Hamiltonian has disjoint support in $t$ from the support of the nonelliptic part.

We will be using four cutoff functions,

$$
\psi_{1}(t), \psi_{2}(t), \psi(t), \text { and } \chi(t):[0,1] \rightarrow[0,1]
$$

satisfying the following properties (see Figure 7):

(i) $\psi_{1}(0)=\psi_{2}(0)=\psi(0)=\chi(0)=0, \quad \psi_{1}(1)=\psi_{2}(1)=\psi(1)=\chi(1)=1$;

(ii) $\psi_{1}^{\prime}, \psi^{\prime}$, and $\chi^{\prime}$ are all nonnegative;

(iii) $\operatorname{supp} \psi_{1}^{\prime} \subset[0,1 / 4], \operatorname{supp} \chi^{\prime} \subset[1 / 4,1 / 2]$,

$\operatorname{supp} \psi^{\prime} \subset[1 / 2,3 / 4]$, and $\operatorname{supp} \psi^{\prime} \subset[3 / 4,1]$.

Motivated by Proposition 7.3, we construct a family of symplectomorphisms, $\kappa_{z, t}$, satisfying $\kappa_{z, 0}=$ id and $\kappa_{z, 1}=S(z)$, but the elliptic part has support in $t$ disjoint from the hyperbolic part. That is, let $F$ be given as in Proposition 7.1, and let

$$
E(z)=\exp (-J F(z)), \quad K_{t}^{1}=\exp (-t J F(z)),
$$

so that $K_{0}^{1}=$ id, $K_{1}^{1}=E(z)$, and

$$
\frac{d}{d t} K_{t}^{1}=\left(K_{t}^{1}\right)_{*} H_{q^{1}},
$$


3414

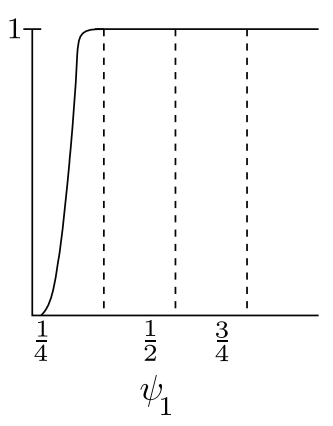

HANS CHRISTIANSON
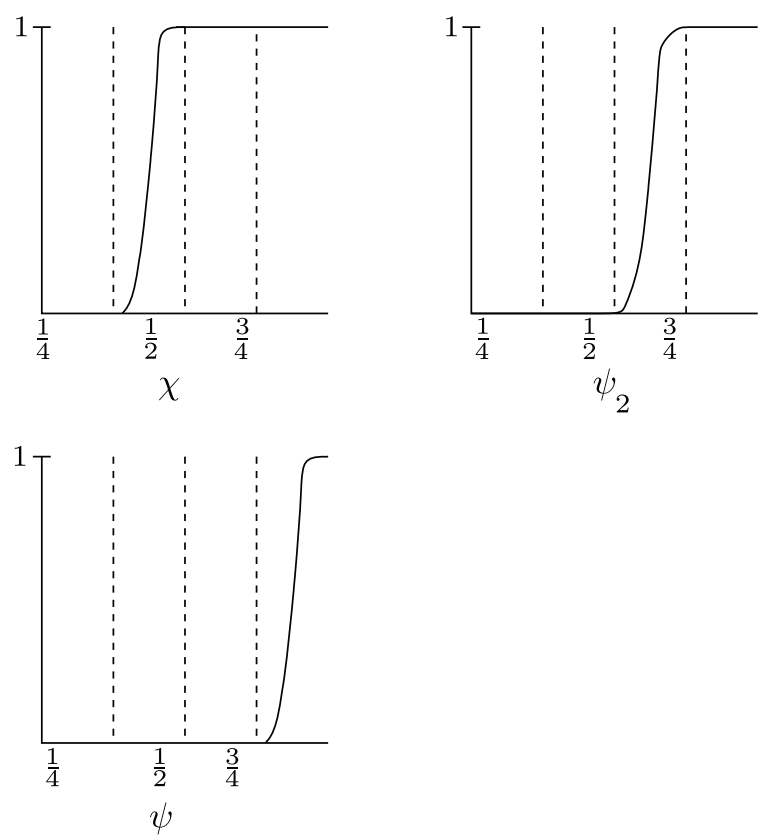

Figure 7 . The cutoff functions $\psi_{1}, \psi_{2}, \psi$, and $\chi$.

where $q^{1}$ is given by (7.11). Here the coefficients $\frac{\operatorname{Im} \lambda_{j}}{2}$ implicitly depend on $z$, but the dimensions of the eigenspaces are constant for $z$ in a neighbourhood of 0 . Let $\widetilde{K}_{t}^{1}$ be defined by

$$
\widetilde{K}_{t}^{1}=K_{\psi_{1}(t)}^{1},
$$

so that $\widetilde{K}_{0}^{1}=$ id, $\widetilde{K}_{1}^{1}=E(z)$, and the chain rule then gives

$$
\begin{aligned}
\frac{d}{d t} \widetilde{K}_{t}^{1} & =\left.\psi_{1}^{\prime}(t) \frac{d}{d \tau} K_{\tau}^{1}\right|_{\tau=\psi_{1}(t)} \\
& =\left.\psi_{1}^{\prime}(t)\left(K_{\tau}^{1}\right)_{*} H_{q^{1}}\right|_{\tau=\psi_{1}(t)} \\
& =\left(\widetilde{K}_{t}^{1}\right)_{*} H_{\psi_{1}^{\prime}(t) q^{1}} .
\end{aligned}
$$

We introduce an "artificial hyperbolic" transformation which will temporarily replace the elliptic part by setting

$$
q_{a h}=\sum_{j=2 h_{h c}+n_{h r+}+n_{h r-}+1}^{2 h_{h c}+n_{h r+}+n_{h r-}+n_{e}} 2 x_{j} \xi_{j},
$$

defining $K_{a h}=\exp \left(H_{q_{a h}}\right)$, and

$$
\widetilde{S}(z)=K_{a h}^{-1} \circ E(z)^{-1} \circ S(z),
$$

so that $\widetilde{S}(z)$ satisfies the assumptions of Proposition 7.3 near $z=0$. From Proposition 7.3 there is a family $\kappa_{z, t}^{1}$ satisfying $\kappa_{z, 0}^{1}=\mathrm{id}, \kappa_{z, 1}^{1}=\widetilde{S}(z)$, and

$$
\frac{d}{d t} \kappa_{z, t}^{1}=\left(\kappa_{z, t}^{1}\right)_{*} H_{\tilde{q}_{z, t}},
$$


where now

$$
\tilde{q}_{z, t}=\left\langle B_{z, t}(x, \xi) x, \xi\right\rangle
$$

for $B_{z, t}$ satisfying (7.14). Let

$$
\tilde{\kappa}_{z, t}=\kappa_{z, \psi(t)}^{1}
$$

so that $\tilde{\kappa}_{z, 0}=\mathrm{id}, \tilde{\kappa}_{z, 1}=\widetilde{S}(z)$, and

$$
\begin{aligned}
\frac{d}{d t} \tilde{\kappa}_{z, t} & =\left.\psi^{\prime}(t) \frac{d}{d \tau} \kappa_{z, \tau}^{1}\right|_{\tau=\psi(t)} \\
& =\left.\psi^{\prime}(t)\left(\kappa_{z, \tau}^{1}\right)_{*} H_{\tilde{q}_{z, \tau}}\right|_{\tau=\psi(t)} \\
& =\left(\tilde{\kappa}_{z, t}\right)_{*} H_{\psi^{\prime}(t) \tilde{q}_{z, \psi(t)}}
\end{aligned}
$$

Let $K_{t}^{2}=\exp \left(t H_{q_{a h}}\right)$ and $\widetilde{K}_{t}^{2}=K_{\psi_{2}(t)}^{2}$, so that $\widetilde{K}_{0}^{2}=\mathrm{id}, \widetilde{K}_{1}^{2}=K_{a h}$, and

$$
\frac{d}{d t} \widetilde{K}_{t}^{2}=\left(\widetilde{K}_{t}^{2}\right)_{*} H_{\psi_{2}^{\prime}(t) q_{a h}}
$$

Finally, let

$$
\kappa_{z, t}=\widetilde{K}_{t}^{1} \circ \widetilde{K}_{t}^{2} \circ \tilde{\kappa}_{z, t} .
$$

Unraveling the definitions, we have $\kappa_{z, t}$ satisfying

$$
\begin{aligned}
& \text { (i) } \kappa_{z, 0}=\text { id }, \quad \kappa_{z, 1}=S(z) \\
& \text { (ii) } \kappa_{z, t}=\left\{\begin{array}{l}
\widetilde{K}_{t}^{1}, \quad 0 \leq t \leq 1 / 4 \\
E(z), 1 / 4 \leq t \leq 1 / 2 \\
E(z) \circ \widetilde{K}_{t}^{2}, 1 / 2 \leq t \leq 3 / 4 \\
E(z) \circ K_{a h} \circ \widetilde{\kappa}_{z, t}, 3 / 4 \leq t \leq 1 .
\end{array}\right.
\end{aligned}
$$

If we compose a smooth function $a$ with $\kappa_{z, t}$, we have

$$
\begin{aligned}
& \frac{d}{d t} \kappa_{z, t}^{*} a=\left\{\begin{array}{l}
\frac{d}{d t} a\left(\widetilde{K}_{t}^{1}\right), \quad 0 \leq t \leq 1 / 4 ; \\
\frac{d}{d t} a(E(z)), \quad 1 / 4 \leq t \leq 1 / 2 ; \\
\frac{d}{d t} a(E(z)) \circ \widetilde{K}_{t}^{2}, \quad 1 / 2 \leq t \leq 3 / 4 ; \\
\frac{d}{d t} a\left(E(z) K_{a h}\right) \circ \tilde{\kappa}_{z, t}, \quad 3 / 4 \leq t \leq 1 ;
\end{array}\right. \\
&=\left\{\begin{array}{l}
\left(H_{\psi_{1}^{\prime}(t) q^{1}} a\right) \circ \widetilde{K}_{t}^{1}, \quad 0 \leq t \leq 1 / 4 ; \\
0,1 / 4 \leq 1 / 2 ; \\
\left.\left(H_{(E(z)-1}\right)^{\prime} \psi_{2}^{\prime}(t) q_{a h} a\right) \circ E(z) \circ \widetilde{K}_{t}^{2}, \quad 1 / 2 \leq t \leq 3 / 4 ; \\
\left(H_{\left(K_{a h}^{-1}\right) *(E(z)-1) * \psi^{\prime}(t) \tilde{q}_{z, \psi(t)}} a\right) \circ E(z) \circ K_{a h} \circ \tilde{\kappa}_{z, t}, \quad 3 / 4 \leq t \leq 1 .
\end{array}\right.
\end{aligned}
$$

Summing up and using the support properties of $\psi, \psi_{1}$, and $\psi_{2}$, we have

$$
\frac{d}{d t} \kappa_{z, t}=\left(\kappa_{z, t}\right)_{*} H_{\tilde{q}_{z, t}^{2}},
$$

where

$$
\tilde{q}_{z, t}^{2}=\left(E(z)^{-1} K_{a h}^{-1}\right)^{*} \psi^{\prime}(t) \tilde{q}_{z, \psi(t)}+\left(E(z)^{-1}\right)^{*} \psi_{1}^{\prime}(t) q^{1}+\psi_{2}^{\prime}(t) q_{a h} .
$$


We record for later use that since $\psi=\psi^{\prime}=0$ and $\psi_{2}=\psi_{2}^{\prime}=0$ on the support of $\chi^{\prime}$, we have for $t \in \operatorname{supp} \chi^{\prime}$,

$$
\kappa_{z, t}=E(z)=\left(\begin{array}{ccc}
\operatorname{id}_{h+} & 0 & 0 \\
0 & -\operatorname{id}_{h-} & 0 \\
0 & 0 & E \tilde{(z)}
\end{array}\right),
$$

where id $h_{h+}$ is the identity in $x_{j}$ and $\xi_{j}$ for

$$
1 \leq j \leq 2 n_{h c}+n_{h r+},
$$

$\operatorname{id}_{h-}$ is the identity in $x_{j}$ and $\xi_{j}$ for

$$
2 n_{h c}+n_{h r+}+1 \leq j \leq 2 n_{h c}+n_{h r+}+n_{h r-},
$$

and $\tilde{E(z)}$ is a $z$-dependent family of elliptic symplectic transformations in the variables $x_{j}$ and $\xi_{j}$ for

$$
2 n_{h c}+n_{h r+}+n_{h r-}+1 \leq j \leq 2 n_{h c}+n_{h r+}+n_{h r-}+n_{e} .
$$

8.3. Step 2: Conjugation of evolution equations. For Step 2, we introduce the following notation. By $\left(X_{\text {hyp }}, \Xi_{\text {hyp }}\right)$ and $\left(X_{\text {ell }}, \Xi_{\text {ell }}\right)$ we mean the symplectic variables in the subspace associated to the hyperbolic and elliptic parts of $d S(0,0)$, respectively. In our notation,

$$
X_{\text {hyp }}=\left(X_{1}, \ldots, X_{n-n_{e}-1}\right), \quad \Xi_{\text {hyp }}=\left(\Xi_{1}, \ldots, \Xi_{n-n_{e}-1}\right),
$$

and

$$
X_{\text {ell }}=\left(X_{n-n_{e}}, \ldots, X_{n-1}\right), \Xi_{\text {ell }}=\left(\Xi_{n-n_{e}}, \ldots, \Xi_{n-1}\right) .
$$

The assumption that $\gamma$ is semi-hyperbolic amounts to saying that $n-1-n_{e} \geq 1$, or that $X_{\text {hyp }}$ are nontrivial variables. For a vector $Y \in \mathbb{R}^{n-1}$, we also define

$$
\begin{aligned}
|Y|_{\text {hyp }}^{2} & =\sum_{j=1}^{n-n_{e}-1} Y_{j}^{2} \text { and } \\
|Y|_{\text {ell }}^{2} & =\sum_{j=n-n_{e}}^{n-1} Y_{j}^{2},
\end{aligned}
$$

where as usual $n_{e}=n-1-2 n_{h c}-n_{h r+}-n_{h r-}$. If

$$
\langle B \cdot, \cdot\rangle: \mathbb{R}^{n-1} \times \mathbb{R}^{n-1} \rightarrow \mathbb{C}
$$

is a bilinear form, we will also use the notation

$$
\left\langle B Y,\left(Z_{\mathrm{hyp}}, i Z_{\mathrm{ell}}\right)\right\rangle=\sum_{j=1}^{n-1} \sum_{k=1}^{n-n_{e}-1} B^{j k} Y_{j} Z_{k}+i \sum_{j=1}^{n-1} \sum_{k=n-n_{e}}^{n-1} B^{j k} Y_{j} Z_{k} .
$$

Let $W(z)=M(z)^{-1}$ as above and let $Q_{z, t}=\operatorname{Op}_{h}^{w}\left(\tilde{q}_{z, t}^{2}\right)$ for $\tilde{q}_{z, t}^{2}$ in the form (8.5). Again by modifying the proofs of the Egorov theorem and its converse, there is $W^{z}(t)$ and $W_{z, 0}$ unitary satisfying (8.2) with this choice of $Q_{z, t}$ so that $W^{z}(1)=W(z)$. As in 9 , but with $W^{z}$ instead of $M^{z}$, if we conjugate $W^{z}(t)$ satisfying (8.2) (8.3) in a way which is independent of $t$, we get a new equation with a conjugated $Q_{z, t}$. That is, with $T_{h, \tilde{h}}$ defined in (6.1), let

$$
W^{z, 1}(t)=T_{h, \tilde{h}} W^{z}(t) T_{h, \tilde{h}}^{-1}
$$


and observe that $W^{z, 1}(t)$ satisfies

$$
\begin{aligned}
h D_{t} W^{z, 1}-Q_{z, t}^{1} W^{z, 1} & =0,0 \leq t \leq 1, \\
W^{z, 1}(0) & =T_{h, \tilde{h}} W_{z, 0} T_{h, \tilde{h}}^{-1}
\end{aligned}
$$

for $Q_{z, t}^{1}=T_{h, \tilde{h}} Q_{z, t} T_{h, \tilde{h}}^{-1}$.

We define the escape function $G$ in the new coordinates by

$$
\begin{aligned}
G(X, \Xi) & =\frac{1}{2} \log \left(\frac{1+|X|_{\text {hyp }}^{2}}{1+|\Xi|_{\text {hyp }}^{2}}\right)+i \frac{1}{2}\left(\left|X_{\text {ell }}\right|^{2}-\left|\Xi_{\text {ell }}\right|^{2}\right) \\
& =: G_{1}+i G_{2} .
\end{aligned}
$$

Here we have added an imaginary term to the definition of $G$. Observe

$$
\exp \left(i \mathrm{Op}_{\tilde{h}}^{w}\left(G_{2}\right)\right)
$$

is unitary. As mentioned in the introduction to this section, this is used to control the nonlinear interactions between the hyperbolic and elliptic variables in a Poisson bracket later in the proof.

The real part of $G, G_{1}$, satisfies

$$
\left|\partial_{X}^{\alpha} \partial_{\Xi}^{\beta} G_{1}(X, \Xi)\right| \leq C_{\alpha \beta}\langle X\rangle^{-|\alpha|}\langle\Xi\rangle^{-|\beta|}, \quad \text { for }(\alpha, \beta) \neq(0,0),
$$

and since $\langle X\rangle^{2}\langle\Xi\rangle^{-2}$ is an order function, $\operatorname{Re} G$ satisfies the assumptions of Chr1, Lemma 2.1]. Thus we can construct the operators $e^{ \pm s \chi(t) G^{w}}$, where $G^{w}$ is the $\tilde{h}$-Weyl quantization of $G$, and doing so we may define

$$
\widetilde{W}(t)=e^{-s \chi G^{w}} W^{z, 1}(t) e^{s \chi G^{w}} .
$$

Similar to $₫ 6$, $\widetilde{W}$ satisfies the evolution equation

$$
\begin{aligned}
h D_{t} \widetilde{W}-\widetilde{Q}_{z, t} \widetilde{W} & =\frac{h}{i} s \chi^{\prime}(t) e^{-s \chi G^{w}}\left[W^{z, 1}, G^{w}\right] e^{s \chi G^{w}}, 0 \leq t \leq 1, \\
\widetilde{W}(0) & =e^{-s \chi(0) G^{w}} T_{h, \tilde{h}} W_{z, 0} T_{h, \tilde{h}}^{-1} e^{s \chi(0) G^{w}},
\end{aligned}
$$

where

$$
\widetilde{Q}_{z, t}=e^{-s \chi G^{w}} Q_{z, t}^{1} e^{s \chi G^{w}} .
$$

The definition of $W^{z, 1}$ together with modifying the proof of the Egorov theorem to the 2-parameter setting (using [Chr1, Lemma 2.5] to estimate the commutators) implies

$$
\begin{aligned}
\chi^{\prime}(t)\left[W^{z, 1}, G^{w}\right] & =\chi^{\prime}(t) T_{h, \tilde{h}}\left[W^{z}, T_{h, \tilde{h}}^{-1} G^{w} T_{h, \tilde{h}}\right] T_{h, \tilde{h}}^{-1} \\
& =\chi^{\prime}(t) T_{h, \tilde{h}} \mathrm{Op}_{h}^{w}\left(\kappa_{z, t}^{*} \widetilde{G}-\widetilde{G}+\mathcal{O}\left(h^{1 / 2} \tilde{h}^{3 / 2}\right)\right) W^{z} T_{h, \tilde{h}}^{-1},
\end{aligned}
$$

where

$$
\widetilde{G}(x, \xi)=G\left((\tilde{h} / h)^{\frac{1}{2}}(x, \xi)\right) \in \mathcal{S}_{\frac{1}{2}}^{-\infty, 0,0} \text { microlocally. }
$$

From (8.6) and the definition of $G$,

$$
\operatorname{Re} \kappa_{z, t}^{*} \widetilde{G}=\operatorname{Re} \widetilde{G}
$$


on $\operatorname{supp} \chi^{\prime}$. Hence, using Lemma 2.2 and the modification of the Egorov theorem to the 2-parameter setting, there is a symbol $e_{t} \in \mathcal{S}_{0}^{-\infty,-1 / 2,-3 / 2}$ such that

$$
\begin{aligned}
\operatorname{Im} \frac{h}{i} & s \chi^{\prime}(t) e^{-s \chi G^{w}}\left[W^{z, 1}, G^{w}\right] e^{s \chi G^{w}} \\
& =\operatorname{Im} \frac{h}{i} s \chi^{\prime}(t)\left(\operatorname{Op}_{\tilde{h}}\left(e_{t}\right)+\frac{\tilde{h}}{i} s \chi^{\prime}(t) G^{w} \mathrm{Op}_{\tilde{h}}\left(\left\{e_{t}, G\right\}\right)+\mathcal{O}\left(h^{1 / 2} \tilde{h}^{7 / 2}\right)\right) \\
& =\mathcal{O}\left(h^{3 / 2} \tilde{h}^{3 / 2}\right) .
\end{aligned}
$$

8.4. Step 3: Estimation of $\widetilde{Q}_{z, t}$. We want to gain some knowledge of $\widetilde{Q}_{z, t}$. For that we use the techniques from the proof of Theorem 1 in Chr1 together with the necessary modifications discussed in the introduction. We summarize the content of this step in the following lemma:

Lemma 8.1. For $\widetilde{Q}_{z, t}$ as defined above, we have the estimate

$$
-\operatorname{Im}\left\langle\widetilde{Q}_{z, t} u, u\right\rangle \geq \psi^{\prime}(t) \frac{h \tilde{h}}{C}\|u\|^{2},
$$

for any $u \in L^{2}\left(\mathbb{R}^{n-1}\right)$.

The idea is that the conjugated $\widetilde{Q}_{z, t}$ is $Q_{z, t}^{1}$ to leading order, which is selfadjoint, and the second-order term is roughly the quantization of

$$
\frac{\tilde{h}}{i} H_{q} G
$$

for a quadratic form $q$. But then [Chr1, Theorem 4] says that for the quadratic forms in which we are interested we can make $H_{q} G$ into a positive definite quadratic form, and there are linear symplectic coordinates in which $H_{q} G$ is almost the harmonic oscillator $\sum_{j} x_{j}^{2}+\xi_{j}^{2}$.

Let $U$ be a neighbourhood of $(0,0), U \subset T^{*} \mathbb{R}^{n-1}$, and assume

$$
U \subset U_{\epsilon / 2}:=\left\{(x, \xi):|(x, \xi)|<\frac{\epsilon}{2}\right\}
$$

for $\epsilon>0$. We assume throughout that we are working microlocally in $U_{\epsilon}$. With $\tilde{h}$ small (fixed later in the proof), we have done the following rescaling:

$$
X:=(\tilde{h} / h)^{\frac{1}{2}} x, \quad \Xi:=(\tilde{h} / h)^{\frac{1}{2}} \xi .
$$

We assume for the remainder of the proof that $|(X, \Xi)| \leq(\tilde{h} / h)^{\frac{1}{2}} \epsilon$. We used the unitary operator $T_{h, \tilde{h}}$ defined in (6.1) to introduce the second parameter into $Q_{z, t}$ to get

$$
Q_{z, t}^{1}=T_{h, \tilde{h}} Q_{z, t} T_{h, \tilde{h}}^{-1}
$$

as above. We now use the support properties of the cutoff functions chosen in Step 1. That is, since $\psi_{1}^{\prime}=0$ on supp $\chi$, after a linear symplectic change of variables, we write

$$
\begin{aligned}
\chi(t) Q_{z, t}^{1} & =\chi(t) Q_{z, t}^{2} \\
& :=\chi(t) T_{h, \tilde{h}} \mathrm{Op}_{h}\left(\psi^{\prime}(t) \tilde{q}_{z, \psi(t)}+\left(K_{a h} E(z)\right)^{*} \psi_{2}^{\prime}(t) q_{a h}\right) T_{h, \tilde{h}}^{-1},
\end{aligned}
$$


where $\tilde{q}_{z, t}=\left\langle B_{z, t} x, \xi\right\rangle$ as defined in Step 1. According to (8.5), the principal symbol of $Q_{z, t}^{2}$ on $\operatorname{supp} \chi$ is, after a linear symplectic change of variables in the elliptic variables alone,

$$
\begin{aligned}
q_{z, t}^{2}(X, \Xi)= & q_{z, t}^{3}(X, \Xi)+q_{a h}\left((h / \tilde{h})^{\frac{1}{2}}(X, \Xi)\right) \\
= & \psi^{\prime}(t)\left\langle B_{z, \psi(t)}\left((h / \tilde{h})^{\frac{1}{2}}(X, \Xi)\right)(h / \tilde{h})^{\frac{1}{2}} X,(h / \tilde{h})^{\frac{1}{2}} \Xi\right\rangle \\
& \quad+\psi_{2}^{\prime}(t) \sum_{j=2 h_{h c}+n_{h r+}+n_{h r-}+1}^{2 h_{h c}+n_{h r+}+n_{h r-}+n_{e}}(h / \tilde{h}) 2 X_{j} \Xi_{j},
\end{aligned}
$$

and $q_{z, t}^{2} \in \mathcal{S}_{-\frac{1}{2}}^{-\infty, 0,0}$ microlocally. Here we have written

$$
q_{z, t}^{3}(X, \Xi)=\psi^{\prime}(t)\left\langle B_{z, \psi(t)}\left((h / \tilde{h})^{\frac{1}{2}}(X, \Xi)\right)(h / \tilde{h})^{\frac{1}{2}} X,(h / \tilde{h})^{\frac{1}{2}} \Xi\right\rangle
$$

to help clear up some of the notation. We have

$$
\left|\partial_{X, \Xi}^{\alpha} q_{z, t}^{2}\right| \leq C_{\alpha}(h / \tilde{h})^{|\alpha| / 2}
$$

for $(X, \Xi) \in U_{(\tilde{h} / h)^{\frac{1}{2}} \epsilon}$ by [Chr1, Lemma 2.4].

Now $\operatorname{Re}\left\{G, q_{a h}\left((h / \tilde{h})^{1 / 2}(X, \Xi)\right)\right\}=0$, so to find the real part of $H_{q_{z, t}^{2}} G$, we need only calculate $\operatorname{Re} H_{q_{z, t}^{3}} G$. For $|(X, \Xi)| \leq(\tilde{h} / h)^{\frac{1}{2}} \epsilon$ we have with $G$ as above in (8.7),

$$
\begin{array}{rl}
H_{q_{z, t}^{3}} & G(X, \Xi) \\
= & (h / \tilde{h}) \psi^{\prime}(t)\left[\left\langle B_{z, \psi(t)} X, \frac{\partial}{\partial X}\right\rangle-\left\langle B_{z, \psi(t)} \frac{\partial}{\partial \Xi}, \Xi\right\rangle\right] G(X, \Xi) \\
& +(h / \tilde{h})^{\frac{3}{2}} \psi^{\prime}(t)\left[\sum_{j=1}^{n-1}\left\langle\frac{\partial}{\partial \Xi_{j}} B_{z, \psi(t)}(\cdot, \cdot) X, \Xi\right\rangle \frac{\partial}{\partial X_{j}} G(X, \Xi)\right] \\
& -(h / \tilde{h})^{\frac{3}{2}} \psi^{\prime}(t)\left[\sum_{j=1}^{n-1}\left\langle\frac{\partial}{\partial X_{j}} B_{z, \psi(t)}(\cdot, \cdot) X, \Xi\right\rangle \frac{\partial}{\partial \Xi_{j}} G(X, \Xi)\right] .
\end{array}
$$

Now owing to Lemma [Chr1, Lemma 2.5] and (8.14) we have microlocally to leading order in $h$ :

$$
\operatorname{Re} \operatorname{ad}_{G^{w}}^{k}\left(Q_{z, t}^{2}\right)=\mathcal{O}_{L^{2} \rightarrow L^{2}}\left(h \tilde{h}^{k-1}\right)
$$

and, in particular,

$$
i \operatorname{Im}\left[Q_{z, t}^{2}, G^{w}\right]=-i \tilde{h} \operatorname{Re} \operatorname{Op}_{\tilde{h}}^{w}\left(H_{q_{z, t}^{2}} G\right)+\mathcal{O}\left(h^{3 / 2} \tilde{h}^{3 / 2}\right) .
$$

Estimating the real part of the errors (8.16) - 8.17), we get

$$
\begin{gathered}
\operatorname{Re}(h / \tilde{h})^{\frac{3}{2}}\left[\sum_{j=1}^{n-1}\left\langle\frac{\partial}{\partial \Xi_{j}} B_{z, \psi(t)}(\cdot, \cdot) X, \Xi\right\rangle \frac{\partial}{\partial X_{j}} G(X, \Xi)\right] \\
=(h / \tilde{h})^{\frac{3}{2}} \frac{1}{1+\left|X_{\text {hyp }}\right|^{2}} \mathcal{O}\left(|\Xi \| X|\left|X_{\text {hyp }}\right|\right),
\end{gathered}
$$


and analogously for (8.17). At $(0,0), B_{z, \psi(t)}$ is positive definite and block diagonal of the form (7.14), so we compute:

$$
\begin{aligned}
\left|\left\langle B_{\psi(t)}(0,0) X,\left(\frac{X_{\text {hyp }}}{1+\left|X_{\text {hyp }}\right|^{2}}, i X_{\text {ell }}\right)\right\rangle\right| & \geq C^{-1}\left(\frac{\left|X_{\text {hyp }}\right|^{2}}{1+\left|X_{\text {hyp }}\right|^{2}}+\left|X_{\text {ell }}\right|^{2}\right) \\
& =C^{-1} \frac{|X|^{2}+\left|X_{\text {hyp }}\right|^{2}\left|X_{\text {ell }}\right|^{2}}{1+\left|X_{\text {hyp }}\right|^{2}} .
\end{aligned}
$$

Hence

$$
\begin{gathered}
\operatorname{Re} \frac{1}{1+\left|X_{\text {hyp }}\right|^{2}} \mathcal{O}\left(|\Xi||X|\left|X_{\text {hyp }}\right|\right) \frac{\left\langle B_{\psi(t)}(0,0) X,\left(\frac{X_{\text {hyp }}}{1+\left|X_{\text {hyp }}\right|^{2}}, i X_{\text {ell }}\right)\right\rangle}{\left|\left\langle B_{\psi(t)}(0,0) X,\left(\frac{X_{\text {hyp }}}{1+\left|X_{\text {hyp }}\right|^{2}}, i X_{\text {ell }}\right)\right\rangle\right|} \\
=\operatorname{Re}\left\langle B_{\psi(t)}(0,0) X,\left(\frac{X_{\text {hyp }}}{1+\left|X_{\text {hyp }}\right|^{2}}, i X_{\text {ell }}\right)\right\rangle \mathcal{O}(|\Xi|),
\end{gathered}
$$

and analogously for (8.17). Now we expand $B_{z, \psi(t)}$ in a Taylor approximation about $(0,0)$ to get

$$
\begin{gathered}
\operatorname{Re} H_{q_{z, t}^{3}} G=\operatorname{Re}(h / \tilde{h}) \psi^{\prime}(t)\left[\left\langle B_{z, \psi(t)}(0,0) X,\left(\frac{X_{\text {hyp }}}{1+|X|_{\text {hyp }}^{2}}, i X_{\text {ell }}\right)\right\rangle\right. \\
\left.+\operatorname{Re}(h / \tilde{h})^{\frac{1}{2}} \mathcal{O}\left(\frac{|X|\left|X_{\text {hyp }}\right|}{1+\left|X_{\text {hyp }}\right|^{2}}|(X, \Xi)|\right)\right] \\
+\operatorname{Re}(h / \tilde{h}) \psi^{\prime}(t)\left[\left\langle B_{z, \psi(t)}(0,0) \Xi,\left(\frac{\Xi_{\text {hyp }}}{1+|\Xi|_{\text {hyp }}^{2}}, i \Xi_{\text {ell }}\right)\right\rangle\right. \\
\left.+\operatorname{Re}(h / \tilde{h})^{\frac{1}{2}} \mathcal{O}\left(\frac{|\Xi|\left|\Xi_{\text {hyp }}\right|}{1+\left|\Xi_{\text {hyp }}\right|^{2}}|(X, \Xi)|\right)\right],
\end{gathered}
$$

which, from (8.20), is

$$
\begin{aligned}
\operatorname{Re} H_{q_{z, t}^{3}} G=\operatorname{Re}(h / \tilde{h}) \psi^{\prime}(t)\left\langle B_{z, \psi(t)}(0,0) X,\left(\frac{X_{\text {hyp }}}{1+|X|_{\text {hyp }}^{2}}, i X_{\text {ell }}\right)\right\rangle \\
\cdot\left(1+(h / \tilde{h})^{\frac{1}{2}} \mathcal{O}(|\Xi|)\right) \\
+\operatorname{Re}(h / \tilde{h}) \psi^{\prime}(t)\left\langle B_{z, \psi(t)}(0,0) \Xi,\left(\frac{\Xi_{\text {hyp }}}{1+|\Xi|_{\text {hyp }}^{2}}, i \Xi_{\text {ell }}\right)\right\rangle \\
\cdot\left(1+(h / \tilde{h})^{\frac{1}{2}} \mathcal{O}(|X|)\right) .
\end{aligned}
$$

Now since $B_{z, \psi(t)}(0,0)$ is block diagonal of the form (7.14), Chr1, Theorem 4] yields a linear symplectomorphism $\kappa_{1}$ such that

$$
\begin{aligned}
\operatorname{Re} \kappa_{1}^{*}\left(H_{q_{z, t}^{2}}(G)\right)= & (h / \tilde{h}) \psi^{\prime}(t)\left[\frac{\sum_{j=1}^{n-n_{e}-1} r_{j}^{-2} X_{j}^{2}}{1+|M X|^{2}}\left(1+(h / \tilde{h})^{\frac{1}{2}} \mathcal{O}(|\Xi|)\right)\right. \\
& \left.+\frac{\sum_{j=1}^{n-n_{e}-1} r_{j}^{-2} \Xi_{j}^{2}}{1+\left|M^{\prime} \Xi\right|^{2}}\left(1+(h / \tilde{h})^{\frac{1}{2}} \mathcal{O}(|X|)\right)\right]
\end{aligned}
$$


where $M$ and $M^{\prime}$ are nonsingular. Thus, since $\chi(t) \psi_{1}^{\prime}(t)=0$,

$$
\begin{aligned}
\operatorname{Im} \widetilde{Q}_{z, t}= & \operatorname{Im} s \chi(t)\left[Q_{z, t}^{2}, G^{w}\right]+s \chi(t) E_{1}^{w}+s^{2} \chi(t)^{2} E_{2}^{w} \\
= & -s h \chi(t)\left(A_{1}\left(1+E_{0}\right)+A_{2}\left(1+E_{0}^{\prime}\right)\right)^{w} \\
& +s \chi(t) E_{1}^{w}+s^{2} \chi(t)^{2} E_{2}^{w},
\end{aligned}
$$

with $E_{0}, E_{0}^{\prime}=\mathcal{O}(\epsilon), E_{1}=\mathcal{O}\left(h^{3 / 2} \tilde{h}^{3 / 2}\right), E_{2}=\mathcal{O}(h \tilde{h})$, and $\left(A_{1}+A_{2}\right)^{w}=: A^{w}=$ $\mathrm{Op}_{\tilde{h}}^{w}(A)$ for

$$
A(X, \Xi)=\psi^{\prime}(t)\left(\kappa_{1}^{-1}\right)^{*}\left(\frac{\sum_{j=1}^{n-n_{e}-1} r_{j}^{-2} X_{j}^{2}}{1+|M X|^{2}}+\frac{\sum_{j=1}^{n-n_{e}-1} r_{j}^{-2} \Xi_{j}^{2}}{1+\left|M^{\prime} \Xi\right|^{2}}\right) .
$$

Using the Egorov theorem, there is a unitary $h$-FIO $F_{1}$ quantizing $\kappa_{1}^{-1}$ so that

$$
\tilde{A}:=F_{1} \mathrm{Op}_{\tilde{h}}^{w}(A) F_{1}^{-1}=\mathrm{Op}_{\tilde{h}}^{w}\left(\kappa_{1}^{*} A\right)+\mathcal{O}\left(\tilde{h}^{2}\right) .
$$

We claim that for $\tilde{h}$ sufficiently small and $\tilde{v}$ smooth,

$$
\left\langle\tilde{A}^{w} \tilde{v}, \tilde{v}\right\rangle \geq \frac{\tilde{h}}{C}\|\tilde{v}\|^{2}
$$

for some constant $C>0$, which is essentially the lower bound for the harmonic oscillator $\tilde{h}^{2} D_{X}^{2}+X^{2}$. It suffices to prove this inequality for individual $j$, which is the content of [Chr1, Lemma 5.1]. As $F_{1}$ is unitary, setting $\tilde{v}=F_{1} \tilde{u}$ for $\tilde{u}$ smooth gives

$$
\begin{aligned}
\left\langle A^{w} \tilde{u}, \tilde{u}\right\rangle & \geq \frac{\tilde{h}}{C}\|\tilde{u}\|^{2}-\mathcal{O}\left(\tilde{h}^{2}\right)\|\tilde{u}\|^{2} \\
& \geq \frac{\tilde{h}}{C^{\prime}}\|\tilde{u}\|^{2},
\end{aligned}
$$

for $\tilde{h}>0$ sufficiently small.

Now fix $\tilde{h}>0$ and $|s|>0$ sufficiently small so that the estimate (8.23) holds and the errors $E_{1}$ and $E_{2}$ satisfy

$$
\left\|s h A^{w} \tilde{u}\right\|_{L^{2}} \gg\left\|s E_{1}^{w} \tilde{u}\right\|_{L^{2}}+\left\|s^{2} E_{2}^{w} \tilde{u}\right\|_{L^{2}},
$$

and fix $\epsilon>0$ sufficiently small that the errors $\left|E_{0}\right|,\left|E_{0}\right|^{\prime} \ll 1$, independent of $h>0$.

For $\tilde{u}$ a smooth function with wavefront set contained in $U$, we now have

$$
\begin{aligned}
-\operatorname{Im}\left\langle\widetilde{Q}_{z, t} \tilde{u}, \tilde{u}\right\rangle & \geq \psi^{\prime}(t) \chi(t) \frac{h \tilde{h}}{C}\|\tilde{u}\|^{2} \\
& =\psi^{\prime}(t) \frac{h \tilde{h}}{C}\|\tilde{u}\|^{2},
\end{aligned}
$$

since $\chi(t) \equiv 1$ on the support of $\psi_{1}^{\prime}(t)$. This is (8.11), the crucial estimate needed for Step 4.

If $\tilde{q}_{z, t}$ is not in the form (7.13), by Proposition 7.3 there is a symplectomorphism $\kappa_{2}$ so that $\kappa_{2}^{*} \tilde{q}_{z, t}$ is of the form (7.13). Using the Egorov theorem to quantize $\kappa_{2}$ as an $h$-FIO $F_{2}$, we get

$$
\mathrm{Op}_{h}^{w}\left(\kappa^{*} \tilde{q}_{z, t}+E_{1}\right)=F^{-1} \widetilde{Q}_{z, t} F,
$$

where $E_{1}=\mathcal{O}\left(h^{2}\right)$ is the error arising from the Egorov theorem. We may then use the previous argument for $\kappa_{2}^{*} q_{z, t}$ getting an additional error of $\mathcal{O}\left(h^{2}\right)$ from the Egorov theorem in (8.11). 
8.5. Step 4: Estimation of $\widetilde{W}$. Let $v \in L^{2}(V)$ with wavefront set sufficiently close to $(0,0)$, and set $\tilde{v}=T_{h, \tilde{h}} v$. Now $\widetilde{W}(t)$ is no longer unitary, so we calculate

$$
\begin{aligned}
\partial_{t}\langle\widetilde{W}(t) \tilde{v}, \widetilde{W}(t) \tilde{v}\rangle & =2\left\langle\partial_{t} \widetilde{W}(t) \tilde{v}, \widetilde{W}(t) \tilde{v}\right\rangle \\
& =\frac{2 i}{h}\left\langle\left(\widetilde{Q}_{z, t}+\mathcal{O}\left(h^{3 / 2} \tilde{h}^{3 / 2}\right)\right) \widetilde{W}(t) \tilde{v}, \widetilde{W}(t) \tilde{v}\right\rangle \\
& =-\frac{2}{h}\left\langle\left(\operatorname{Im} \widetilde{Q}_{z, t}+\mathcal{O}\left(h^{3 / 2} \tilde{h}^{3 / 2}\right)\right) \widetilde{W}(t) \tilde{v}, \widetilde{W}(t) \tilde{v}\right\rangle \\
& \geq C^{-1}\left(\psi^{\prime}(t) \tilde{h}-\mathcal{O}\left(h^{1 / 2} \tilde{h}^{3 / 2}\right)\right)\langle\widetilde{W}(t) \tilde{v}, \widetilde{W}(t) \tilde{v}\rangle .
\end{aligned}
$$

Thus there is a positive constant $C$ such that

$$
\partial_{t}\left(\langle\widetilde{W}(t) \tilde{v}, \widetilde{W}(t) \tilde{v}\rangle e^{-\left(\psi(t) \tilde{h}-\mathcal{O}\left(h^{1 / 2} \tilde{h}^{3 / 2}\right)\right) / C}\right) \geq 0
$$

So

$$
\|\widetilde{W}(t) \tilde{v}\|^{2} \geq e^{\psi(t)\left(\tilde{h}-\mathcal{O}\left(h^{1 / 2} \tilde{h}^{3 / 2}\right)\right) / C}\|\widetilde{W}(0) \tilde{v}\|^{2}
$$

and since $\psi(1)=1$, shrinking $\tilde{h}>0$ if necessary, we have for $0<h \leq h_{0}$ sufficiently small,

$$
\|\widetilde{W}(1) \tilde{v}\| \geq R\|\widetilde{W}(0) \tilde{v}\|, \quad R>1 \text { independent of } 0<h \leq h_{0} .
$$

Now

$$
\begin{aligned}
\widetilde{W}(0) & =e^{-s \chi(0) G^{w}} T_{h, \tilde{h}} W^{z}(0) T_{h, \tilde{h}}^{-1} e^{s \chi(0) G^{w}} \\
& =T_{h, \tilde{h}} W^{z}(0) T_{h, \tilde{h}}^{-1}
\end{aligned}
$$

is unitary, so

$$
\|\widetilde{W}(1) \tilde{v}\| \geq R\|\tilde{v}\|
$$

independent of $0<h \leq h_{0}$.

As in $\$$ let the operators $K^{w}$ be defined by

$$
e^{s K^{w}}=T_{h, \tilde{h}}^{-1} e^{s \chi(1) G^{w}} T_{h, \tilde{h}}=T_{h, \tilde{h}}^{-1} e^{s G^{w}} T_{h, \tilde{h}},
$$

so that

$$
\widetilde{W}(1)=e^{-s K^{w}} M(z)^{-1} e^{s K^{w}},
$$

and Theorem 6 is proved.

Remark 8.2. The error arising at the end of the proof of Theorem 6 from the use of the Egorov theorem is of order $\mathcal{O}\left(h^{2}\right)$ and hence negligible compared to our lower bound of $h$ for $A$. However, the estimate of $A$ is used for the imaginary part of $\widetilde{Q}_{z, t}$, and the error in the Egorov theorem is real, so $\mathcal{O}(h)$ would have been sufficient. This means the analysis above does not strictly depend on using the Weyl calculus.

Remark 8.3. It is interesting to note that the estimate (1.3) depends only on the real parts of the eigenvalues $\lambda_{j}$ above. Unraveling the definitions, the eigenvalues $\lambda_{j}$ are logarithms of the eigenvalues of the linearized Poincaré map $d S(0)$ from above. Then (1.3) depends only on the moduli of the eigenvalues of $d S(0)$ which lie off the unit circle. We interpret this as a quantum analogue of the fact that $d S(0,0)$ is semi-hyperbolic. 


\section{Proof of the MAIN TheOrems}

The Main Theorem follows exactly as the Main Theorem in Chr1 with the corrections in Chr1a. The proofs of Theorems $1^{\prime}$ and $2^{\prime}$ proceed with very little modification. The only things left to do are to prove Theorems 3 and $3^{\prime}$ and indicate how to prove the Main Theorem'.

9.1. Proof of Theorems 3 and 3 '. Owing to $\mathrm{BuZw}$, Lemma A.2], an operatorvalued version of the three-line theorem from complex analysis, we only need to prove that in both cases $\widetilde{Q}(z)$ satisfies a polynomial estimate of the form

$$
\left\|\widetilde{Q}(z)^{-1}\right\|_{L^{2}(X) \rightarrow L^{2}(X)} \leq C h^{-N}
$$

for some $N$ and $z \in[-\epsilon, \epsilon]+i\left(-c_{0} h, c_{0} h\right)$. Then [BuZw Lemma A.2] allows us to conclude that

$$
\left\|\widetilde{Q}(z)^{-1}\right\|_{L^{2}(X) \rightarrow L^{2}(X)} \leq C \frac{\log (1 / h)}{h}, z \in[-\epsilon / 2, \epsilon / 2] .
$$

The operator $P(h)-z$ satisfies a similar estimate microlocally near $\gamma$, so we have to glue the microlocal estimate into the better propagation estimates. We first observe that by choosing $s>0$ sufficiently small in (8.8), for any $\delta>0$ we can take $c_{0}$ sufficiently small to get

$$
\|P(-z) u\| \geq \frac{h^{1+\delta}}{C}\|u\|
$$

for $u$ with wavefront set sufficiently close to $\gamma$.

Choose $\psi_{0} \in \mathcal{C}^{\infty}\left(T^{*} X\right)$ satisfying $\psi_{0} \equiv 1$ near $\gamma$ with small support. Choose $W \in \mathcal{C}^{\infty}\left(T^{*} X\right)$ so that $W \equiv 1$ away from $\gamma$ and $W \psi_{0}=0$. Finally, fix $\epsilon>0$ and choose $\psi_{1} \in \mathcal{C}_{c}^{\infty}\left(T^{*} X\right)$ so that $W \geq \epsilon>0$ on $\operatorname{supp}\left(1-\psi_{1}\right)$. Then

$$
\begin{aligned}
\left\|\psi_{0} u\right\| & \leq C h^{-1-\delta}\left\|(P-z) \psi_{0} u\right\|+\mathcal{O}\left(h^{\infty}\right)\|u\| \\
& \leq C h^{-1-\delta}\|\widetilde{Q}(z) u\|+C h^{-\delta}\|\tilde{\psi} u\|+\mathcal{O}\left(h^{\infty}\right)\|u\|,
\end{aligned}
$$

where $\tilde{\psi} \equiv 1$ on $\mathrm{WF}_{h}\left[P, \psi_{0}\right]$. But then the propagation estimate [Chr2, Lemma 2.4] (trivially modified to the complex case) implies

$$
\|\tilde{\psi} u\| \leq C h^{-1}\|\widetilde{Q}(z) u\|+C\left\|\left(1-\psi_{1}\right) u\right\| .
$$

But $|\operatorname{Im} z| \leq c_{0} h$ implies that $W+\operatorname{Im} z \geq \epsilon-c_{0} h \geq \epsilon / 2$ on $\operatorname{supp}\left(1-\psi_{1}\right)$. Hence

$$
\begin{aligned}
\left\|\left(1-\psi_{1}\right) u\right\|^{2} & \leq C\left\langle(W+\operatorname{Im} z)\left(1-\psi_{1}\right) u,\left(1-\psi_{1}\right) u\right\rangle \\
& =-C \operatorname{Im}\left\langle\widetilde{Q}(z)\left(1-\psi_{1}\right) u,\left(1-\psi_{1}\right) u\right\rangle \\
& =-\operatorname{Im}\left\langle\left(1-\psi_{1}\right)^{*}\left(1-\psi_{1}\right) \widetilde{Q}(z) u, u\right\rangle+\mathcal{O}(h)\|u\|^{2} \\
& \leq C h^{-1}\|\widetilde{Q}(z) u\|^{2}+C h^{1}\|u\|^{2} .
\end{aligned}
$$

Similarly, we use propagation again to estimate

$$
\left\|\left(1-\psi_{0}\right) u\right\|^{2} \leq C h^{-1}\|\widetilde{Q}(z) u\|^{2}+C\left\|\left(1-\psi_{1}\right) u\right\|^{2}+\mathcal{O}\left(h^{\infty}\right)\|u\|^{2}
$$

so that

$$
\begin{aligned}
\|u\| & \leq\left\|\psi_{0} u\right\|+\left\|\left(1-\psi_{0}\right) u\right\| \\
& \leq C h^{-1-\delta}\|\widetilde{Q}(z) u\|+C h^{1 / 2-\delta}\|u\| .
\end{aligned}
$$

Taking $\delta<1 / 2$ yields (9.1). 
9.2. Proof of the main theorem. Let $W^{w}$ be a symbol which is microlocally 1 away from $\gamma$, and for $z \in\left[-\epsilon_{0}, \epsilon_{0}\right]+i\left(-c_{0} h, c_{0} h\right)$, define as in (1.6),

$$
\widetilde{Q}(z):=P(h)-z-i W^{w} .
$$

For the analysis near the boundary, also choose $\psi \in \mathcal{C}^{\infty}(\mathbb{R})$ satisfying (3.6)-3.7).

Let $m_{j}^{ \pm} \in T^{*} X$, for $j=1, \ldots, K$ denote the points where $\gamma$ reflects off the boundary, with $m_{j}^{ \pm}$denoting the point of intersection with the boundary of the outgoing and incoming bicharacteristics, respectively, and let $m_{j}$ be the projection of $m_{j}^{ \pm}$onto $T^{*}(\partial X)$. Let $U_{j} \subset T^{*}(\partial X)$ denote a neighbourhood of $m_{j}$ which is small enough so that a factorization of $P$ as in Lemma 3.1 is possible in a neighbourhood of $U_{j}$. Shrinking $U_{j}$ if necessary, we also assume that the construction in Lemma 3.6 is valid in a neighbourhood of $U_{j}$. That is, if $P$ is factorized as in Lemma 3.1 near $U_{j}$, we write

$$
P=\left(h D_{1}-A_{-}\left(x, h D^{\prime}\right)\right)\left(h D_{1}-A_{+}\left(x, h D^{\prime}\right)\right) \text { near } m_{j},
$$

and there is an operator $A_{b, j}\left(x, h D^{\prime}\right)$ which is noncharacteristic microlocally near $U_{j}$, zero away from $U_{j}$ and commutes with $\left(h D_{1}-A_{+}\left(x, h D^{\prime}\right)\right)$ microlocally near $U_{j}$. By multiplying $A_{b, j}$ by a large constant, assume $A_{b, j} \geq 1$ near $U_{j}$.

Let $\gamma_{ \pm}^{j}$ be a small interval on the outgoing/incoming bicharacteristic near $m_{j}^{ \pm}$, and let $\widetilde{U}_{j} \subset T^{*} X$ be a neighbourhood of $\gamma_{ \pm}^{j}$ such that

$$
\left.\left(\mathrm{WF}_{h}\left(\psi(P) A_{b, j}\right)\right)\right|_{\partial X} \subset \widetilde{U}_{j},
$$

and $\psi(P) A_{b, j} \equiv 1$ on $\gamma_{ \pm}^{j} \cap \widetilde{U}_{j}$. Choose $\chi_{j} \in \mathcal{C}_{c}^{\infty}\left(T^{*} X\right), \chi_{j} \equiv 1$ on $\widetilde{U}_{j}$ with sufficiently small support that

$$
\psi(P) A_{b, j} \geq 1 \text { on } \operatorname{supp} \nabla \chi_{j} \cap \gamma
$$

Finally, set

$$
\chi_{0}=1-\sum_{j} \chi_{j} .
$$

Now for $A \in \Psi_{h, d b}^{0,0}$ as in the statement of the Main Theorem' with wavefront set sufficiently close to $\gamma$, let $A_{0} \in \Psi_{h}^{0,0}$ have wavefront set close to $\gamma$ and satisfy

$$
\begin{aligned}
& A_{0} \equiv 1 \text { on }\left\{\mathrm{WF}_{h} A \backslash\left\{\bigcup_{j} \widetilde{U}_{j}\right\}\right\}, \\
& A_{0} \equiv 1 \text { on }\left(\bigcup_{j=0}^{K} \operatorname{supp} \nabla \chi_{j}\right) \cap \gamma, \\
& A_{0} \equiv 0 \text { elsewhere. }
\end{aligned}
$$

We define $\tilde{A} \in \Psi_{h, d b}^{0,0}$ satisfying

$$
\tilde{A} \geq 1 \text { on } \mathrm{WF}_{h} A
$$

by

$$
\tilde{A}=\chi_{0} A_{0}+\sum_{j} \chi_{j} \psi(P) A_{b, j}
$$


where $\psi$ satisfies (3.6) (3.7). Observe that if $\mathrm{WF}_{h} A$ is sufficiently close to $\gamma$, then $\tilde{A}$ satisfies (9.5). We have $\widetilde{Q}(0) \tilde{A} u=P(h) \tilde{A} u$ since $\mathrm{WF}_{h} a^{w} \cap \mathrm{WF}_{h} \tilde{A}=\emptyset$. But

$$
P(h) \tilde{A} u=[P(h), \tilde{A}] u+\tilde{A} P(h) u,
$$

and we claim

$$
\begin{aligned}
\|[P, \tilde{A}] u\| & =\left\|\left[P, \chi_{0} A_{0}\right] u+\sum_{j}\left(\left[P, \chi_{j} \psi(P) A_{b, j}\right]\right) u\right\| \\
& =\mathcal{O}(h)\|(I-A) u\| .
\end{aligned}
$$

To see this, we observe that for $u \in \mathcal{C}^{\infty}(X) \cap L^{2}(X)$,

$$
\begin{aligned}
{\left[P, \chi_{0} A_{0}\right] u } & +\sum_{j}\left[P, \chi_{j} A_{b . j}\right] \\
=\chi_{0}[ & \left.P, A_{0}\right] u+\left[P, \chi_{0}\right] A_{0} u \\
& +\sum_{j}\left(\chi_{j} \psi(P)\left[P, A_{b, j}\right]+\left[P, \chi_{j}\right] \psi(P) A_{b, j}\right) u .
\end{aligned}
$$

We have

$$
\left\|\left(\left[P, \chi_{0}\right] A_{0}+\sum_{j}\left[P, \chi_{j}\right] \psi(P) A_{b, j}\right) u\right\| \leq C h\|(I-A) u\|
$$

from (9.3) and (9.4). These two conditions also imply

$$
\begin{aligned}
& \mathrm{WF}_{h} \chi_{0}\left[P, A_{0}\right] \cap \gamma=\emptyset \text { and } \\
& \mathrm{WF}_{h} \chi_{j} \psi(P)\left[P, A_{b, j}\right] \cap \gamma=\emptyset,
\end{aligned}
$$

and the symbol of $A_{0}$ is compactly supported away from the boundary, so

$$
\left\|\chi_{0}\left[P, A_{0}\right] u\right\| \leq C h\|(I-A) u\| .
$$

For each $j$, it suffices to consider the remaining terms in local coordinates at the boundary. Fix $j$ and assume we are in the coordinates used in Lemma 3.6 in $U_{j}$ :

$$
\begin{aligned}
& \chi_{j} \psi(P)\left[P, A_{b, j}\left(x, h D^{\prime}\right)\right] \\
& \quad=\chi_{j} \psi(P)\left[\left(h D_{1}-A_{-}\left(x, h D^{\prime}\right)\right)\left(h D_{1}-A_{+}\left(x, h D^{\prime}\right)\right), A_{b, j}\right] \\
& \quad=\quad \chi_{j} \psi(P)\left[\left(h D_{1}-A_{-}\left(x, h D^{\prime}\right)\right), A_{b, j}\left(x, h D^{\prime}\right)\right]\left(h D_{1}-A_{+}\left(x, h D^{\prime}\right)\right),
\end{aligned}
$$

since $A_{b, j}$ commutes with $\left(h D_{1}-A_{+}\left(x, h D^{\prime}\right)\right)$. The principal symbol of

$$
\chi_{j} \psi(P)\left[\left(h D_{1}-A_{-}\left(x, h D^{\prime}\right)\right), A_{b, j}\left(x, h D^{\prime}\right)\right]
$$

is

$$
\frac{h}{i} \chi_{j} \psi\left(\left(\xi_{1}-r^{\frac{1}{2}}\left(x, \xi^{\prime}\right)\right)\left(\xi_{1}+r^{\frac{1}{2}}\left(x, \xi^{\prime}\right)\right)\right)\left\{\left(\xi_{1}+r^{\frac{1}{2}}\left(x, \xi^{\prime}\right)\right), \sigma_{h}\left(A_{b, j}^{+}\right)\left(x, \xi^{\prime}\right)\right\},
$$

which is $\mathcal{O}(h)$ and has $h$-wavefront set away from $\gamma$. Summing over $j$ gives (9.7). 
We now use the control theory arguments from $\mathrm{BuZw}$ and Chr1a, together with Theorem $3^{\prime}$. That is, for $z \in[-1 / 2,1 / 2]$,

$$
\begin{aligned}
\|\tilde{A} u\| & \leq C\left\|\widetilde{Q}(z)^{-1} \widetilde{Q}(z) \tilde{A} u\right\|+\mathcal{O}\left(h^{\infty}\right)\|u\| \\
& =C\left\|\widetilde{Q}(z)^{-1}(\tilde{A}(P(h)-z)+[P(h), \tilde{A}]) u\right\|+\mathcal{O}\left(h^{\infty}\right)\|u\| \\
& \leq C\left\|\widetilde{Q}(z)^{-1} \tilde{A}(P(h)-z) u\right\|+C\left\|\widetilde{Q}(z)^{-1} \varphi[P(h), \tilde{A}] u\right\|+\mathcal{O}\left(h^{\infty}\right)\|u\| \\
& \leq C \frac{\log (1 / h)}{h}\|(P(h)-z) u\|+C \log ^{1 / 2}(1 / h)\|(I-A) u\|+\mathcal{O}\left(h^{\infty}\right)\|u\| .
\end{aligned}
$$

Here we have used that $\widetilde{Q}(z) A=(P(h)-z) A$, (3.6), (3.5) and that $P(h)-z$ is elliptic away from $\{p=z\} \supset \gamma$.

This gives

$$
\begin{aligned}
\|u\| & \leq\|\tilde{A} u\|+\|(I-A) u\| \\
& \leq C \frac{\log (1 / h)}{h}\|(P(h)-z) u\|+C\left(\log ^{1 / 2}(1 / h)+1\right)\|(I-A) u\|+\mathcal{O}\left(h^{\infty}\right)\|u\|,
\end{aligned}
$$

which proves the Main Theorem'.

9.3. The proof of Theorem 2 and Theorem $2^{\prime}$. We are now ready to prove Theorem 2 and Theorem $2^{\prime}$, which indicate that the complex absorption term need only be of size $h$. We first repeat the calculations leading to (9.8) with $P(h)$ replaced by $Q(z)=P(h)-z-i h a^{w}$.

Next, we assume $a^{w}=B^{*} B$ for some nonnegative definite $B \in \Psi^{0}$, so that

$$
\|B u\|^{2}=\langle a u, u\rangle,
$$

and Chr2, Lemma 2.4] implies

$$
\|(1-A) u\| \leq \frac{C}{h}\|Q(z) u\|+C\|B u\|+\mathcal{O}\left(h^{\infty}\right)\|u\| .
$$

Then, again using [Chr2, Lemma 2.4] on the term with $(1-A) u$ in (9.8), all told we have the estimate

$$
\|u\| \leq C \frac{\log (1 / h)}{h}\|Q(z) u\|+C \log ^{1 / 2}(1 / h)\|A u\|+\mathcal{O}\left(h^{\infty}\right)\|u\| .
$$

Finally, to get the estimates (1.5) and (3.3) (and the improvement to a complex neighbourhood), we calculate for $z \in[-1 / 2,1 / 2]$,

$$
\begin{aligned}
\|A u\|^{2} & =\langle a u, u\rangle \\
& =\frac{1}{h} \operatorname{Im}\langle Q(z) u, u\rangle \\
& \leq \frac{1}{h}\|Q(z) u\|\|u\|,
\end{aligned}
$$

so that we have for any $\epsilon>0$,

$$
\begin{aligned}
\log ^{1 / 2}(1 / h)\|A u\| & \leq \log ^{1 / 2}(1 / h)\left(\frac{1}{h}\|Q(z) u\|\|u\|\right)^{1 / 2} \\
& \leq \frac{\log (1 / h)}{2 \epsilon h}\|Q(z) u\|+\epsilon\|u\| .
\end{aligned}
$$

Combining this with (9.8) and taking $\epsilon>0$ sufficiently small yields Theorems 2 and $2^{\prime}$. The improvement to $|\operatorname{Im} z| \leq c h / \log (1 / h)$ follows from taking $c>0$ sufficiently small, since then the order of the perturbation is the same order as the estimate. 


\section{An Applichtion: Quasimodes near Elliptic orbits}

In this section, we show how the techniques of reducing microlocal estimates near a periodic orbit to estimates on an $h$-Fourier integral operator acting microlocally on the Poincaré section via the Quantum Monodromy operator from SjZw1 and $\$ 5$ can be used with the quasimode construction in [SZ] to produce well-localized quasimodes near an elliptic periodic orbit. We also give estimates on the number and location of approximate eigenvalues associated to the quasimodes.

Let $X$ be a smooth, compact manifold, $\operatorname{dim} X=n$, and suppose that $P \in$ $\Psi^{k, 0}(X), k \geq 1$, is a semiclassical pseudodifferential operator of real principal type which is semiclassically elliptic outside a compact subset of $T^{*} X$ as in the introduction. Let $\Phi_{t}=\exp t H_{p}$ be the classical flow of $p$ and assume there is a closed elliptic orbit $\gamma \subset\{p=0\}$. That $\gamma$ is elliptic means if $N \subset\{p=0\}$ is a Poincaré section for $\gamma$ and $S: N \rightarrow S(N)$ is the Poincaré map, then $d S(0,0)$ has eigenvalues all of modulus 1 . We will also need the following nonresonance assumption:

$$
\left\{\begin{array}{l}
\text { if } e^{ \pm i \alpha_{1}}, e^{ \pm i \alpha_{2}}, \ldots, e^{ \pm i \alpha_{k}} \text { are eigenvalues of } d S(0,0), \text { then } \\
\alpha_{1}, \alpha_{2}, \ldots, \alpha_{k} \text { are independent over } \pi \mathbb{Z} .
\end{array}\right.
$$

Finally, we assume that if $\gamma \cap \partial X \neq \emptyset$, then $\gamma$ reflects only transversally off $\partial X$, $\partial X$ is noncharacteristic with respect to $P$, and $P \in \operatorname{Diff}_{h}^{2,0}$.

Under these assumptions, it is well known that there is a family of elliptic closed orbits $\gamma_{z} \subset\{p=z\}$ for $z$ near 0 , with $\gamma_{0}=\gamma$. In this work we consider the following eigenvalue problem for $z$ in a neighbourhood of $z=0$ :

$$
\left\{\begin{aligned}
(P-z) u & =0 \\
\|u\|_{L^{2}(X)} & =1
\end{aligned}\right.
$$

We prove the following theorem.

Theorem 7. For each $m \in \mathbb{Z}, m>1$, and each $c_{0}>0$ sufficiently small, there is a finite, distinct family of values

$$
\left\{z_{j}\right\}_{j=1}^{N(h)} \subset\left[-c_{0} h^{1 / m}, c_{0} h^{1 / m}\right]
$$

and a family of quasimodes $\left\{u_{j}\right\}=\left\{u_{j}(h)\right\}$ with

$$
\mathrm{WF}_{h} u_{j}=\gamma_{z_{j}}
$$

satisfying

$$
\left\{\begin{array}{l}
\left(P-z_{j}\right) u_{j}=\mathcal{O}\left(h^{\infty}\right)\left\|u_{j}\right\|_{L^{2}(X)} \\
\left\|u_{j}\right\|_{L^{2}(X)}=1
\end{array}\right.
$$

Further, for each $m \in \mathbb{Z}, m>1$, there is a constant $C=C\left(c_{0}, 1 / m\right)$ such that

$$
C^{-1} h^{-n(1-1 / m)} \leq N(h) \leq C h^{-n} .
$$

10.1. The model case. We consider the case $n=2$, the first nontrivial dimension. Recall that the model for $p$ near an elliptic periodic orbit is $p \in \mathcal{C}^{\infty}\left(T^{*}\left(\mathbb{S}^{1} \times \mathbb{R}\right)\right)$,

$$
p=\tau+\frac{\alpha}{2}\left(x^{2}+\xi^{2}\right)
$$

with $\alpha>0$ satisfying $\alpha \notin \pi \mathbb{Z}$. Then we study (10.2) for

$$
P=h D_{t}+\frac{\alpha}{2}\left(x^{2}+h^{2} D_{x}^{2}\right)
$$


Let

$$
\begin{aligned}
Q & =\frac{\alpha}{2}\left(x^{2}+h^{2} D_{x}^{2}\right) \\
& =\operatorname{Op}_{h}^{w}\left(\frac{\alpha}{2}\left(x^{2}+\xi^{2}\right)\right) .
\end{aligned}
$$

$Q$ is just $\alpha / 2$ times the harmonic oscillator, so we have

$$
Q v_{k}=h \frac{\alpha}{2}(2 k+1) v_{k}
$$

for

$$
\begin{aligned}
v_{k} & :=h^{-1 / 4} H_{k}\left(x / h^{\frac{1}{2}}\right) e^{-x^{2} /(2 h)}, \\
\left\|v_{k}\right\|_{L^{2}} & =1
\end{aligned}
$$

where $H_{k}$ are the (normalized) Hermite polynomials of degree $k$. Note that $\mathrm{WF}_{h} v_{k}$ $=(0,0)$. Now we make an ansatz of

$$
u=g_{k}(t) v_{k}(x)
$$

for $g_{k}(t)$ to be determined. Plugging $u$ into (10.2) yields

$$
h D_{t} g_{k}+\frac{\alpha}{2} h(2 k+1) g_{k}=z g_{k},
$$

which implies

$$
g_{k}(t)=\exp \left(\frac{i t}{h}\left(z-\frac{\alpha}{2}(2 k+1) h\right)\right) .
$$

Since the spectrum of $h D_{t}$ on $\mathbb{S}^{1}$ is $\{2 \pi m h\}_{m \in \mathbb{Z}}$, we have

$$
z=\frac{\alpha}{2}(2 k+1) h+2 \pi m h .
$$

In the model case, since there is no microlocalization necessary (and, in particular, $p$ is not elliptic at infinity), we actually have a dense spectrum in any interval.

In order to motivate our general construction, we present the same example from the point of view of the monodromy operator. Here we think of $Q-z$ as a $z$-dependent family of operators on $L^{2}(V)$, where $V \subset \mathbb{R}$ is an open neighbourhood of 0 . Then the monodromy operator $M(z)$ is defined microlocally as the time $t=1$ solution to the ordinary differential equation

$$
\left\{\begin{array}{l}
h D_{t} M(z, t)+(Q-z) M(z, t)=0, \\
M(z, 0)=\operatorname{id}_{L^{2}(V) \rightarrow L^{2}(V)} .
\end{array}\right.
$$

Our general technique will be to find eigenfunctions of $M(z)=M(z, 1)$ with eigenvalue 1. Again using $v_{k}$ as in the previous paragraph, we try

$$
M(z, t) v_{k}=e^{-i 2 \pi m t} v_{k},
$$

with $m \in \mathbb{Z}$ so that $M(z, 1) v_{k}=v_{k}$. This yields from (10.6) that

$$
\left(-h 2 \pi m+\frac{\alpha}{2} h(2 k+1)-z\right) v_{k}=0,
$$

which is the same as (10.5). 
10.2. Quasimodes on the Poincaré section. Theorem 5 and the definition of the monodromy operator $M(z)$ motivate us to study the normal form for a family of elliptic symplectomorphisms

$$
S_{z}: W_{1} \rightarrow W_{2}
$$

under the nonresonance condition (10.1) on $d S(0)$, where $W_{1}$ and $W_{2}$ are neighbourhoods of $0 \in \mathbb{R}^{2 n-2}$. We use the standard notation of [ISZ] and write

$$
\begin{aligned}
\imath_{j} & =x_{j}^{2}+\xi_{j}^{2}, \text { and } \\
I_{j} & =\imath_{j}^{w}=x_{j}^{2}+h^{2} D_{x_{j}}^{2} .
\end{aligned}
$$

According to the results of [aSj] and [ISZ, there is a symplectic choice of coordinates near $(x, \xi ; z)=(0,0 ; 0)$ such that

$$
S_{z}=\exp H_{q_{z}}+\mathcal{O}\left((x, \xi ; z)^{\infty}\right),
$$

for

$$
q_{z}=\sum_{j=1}^{n-1} \lambda_{j}(z) \imath_{j}+R\left(z, \imath_{1}, \ldots, \iota_{n-1}\right) .
$$

Here the remainder $R(z, \imath)=\mathcal{O}\left(\imath^{2}\right)$ and the $\lambda_{j}(z)$ are positive and depend smoothly on $z$.

Since we are interested in the operator $(P-z)$, we can factor a $z$ out of the monodromy operator before applying the above construction. That is, if

$$
\mathcal{M}(z)=I_{+}(T(z))
$$

is the absolute quantum monodromy operator, by Corollary 3.12 we have

$$
\mathcal{M}(z)=e^{i T_{0} z / h} \widetilde{\mathcal{M}}(z),
$$

where $T_{0}=T(0)$ is the period of $\gamma_{0}$, and $\widetilde{\mathcal{M}}(z)$ is the propagator for an operator $\tilde{P}(z)$ satisfying the same assumptions as $P-z$. Then the quantum monodromy operator

$$
M(z)=e^{i T_{0} z / h} M_{1}(z),
$$

where $M_{1}(z)$ quantizes the Poincaré map. Hence, using [ISZ, if $M(z)$ is the monodromy operator quantizing $S_{z}$ and

$$
\begin{aligned}
& \text { (i) } z \in\left[-\epsilon_{0} h^{1 / m}, \epsilon_{0} h^{1 / m}\right]+i\left(-c_{0} h, c_{0} h\right), \\
& \text { (ii) } \imath_{j} \leq h^{1 / m}
\end{aligned}
$$

for $m \in \mathbb{Z}, m>1$, then there is a family of unitary $h$-FIOs $V(z)$ such that

$$
M(z)=V(z)^{-1} e^{-i\left(Q(z, h)-T_{0} z\right) / h} V(z)+\mathcal{O}_{L^{2} \rightarrow L^{2}}\left(h^{\infty}\right),
$$

where

$$
\begin{aligned}
Q(z, h) & =\sum_{j=0}^{\infty} h^{j} q_{j}(z, I), \text { with } \\
q_{j}(z, I) & =\mathcal{O}(I)
\end{aligned}
$$

and

$$
q_{0}(z, \imath)=q_{z}(\imath)
$$


Now let $\beta \in \mathbb{N}^{n-1}$ be a multi-index and define

$$
v_{\beta}=c_{\beta} h^{-(n-1) / 4} e^{-|x|^{2} /(2 h)} \prod_{j=1}^{n-1} H_{\beta_{j}}\left(x_{j} / h^{\frac{1}{2}}\right),
$$

with $H_{\beta_{j}}$ the Hermite polynomials as in $\$ 10.1$ and $c_{\beta}$ chosen independent of $h$ to normalize $v_{\beta}$ in $L^{2}\left(\mathbb{R}^{n-1}\right)$. The functions $v_{\beta}$ satisfy

$$
I_{j} v_{\beta}=h\left(2 \beta_{j}+1\right) v_{\beta},
$$

and with $\mathbb{1}=(1, \ldots, 1) \in \mathbb{N}^{n-1}$ we write

$$
I v_{\beta}=h(2 \beta+\mathbb{1}) v_{\beta} .
$$

Hence we have

$$
\begin{aligned}
Q(z, h) v_{\beta} & =\left(\sum_{j=0}^{\infty} h^{j} q_{j}(z, h(2 \beta+\mathbb{1}))\right) v_{\beta} \\
& =: \quad \zeta_{\beta}(z) v_{\beta},
\end{aligned}
$$

where

$$
\zeta_{\beta}(z)=h \sum_{j=1}^{n-1} \lambda_{j}(z)\left(2 \beta_{j}+1\right)+\mathcal{O}\left(h^{2}\right) .
$$

The condition (10.9) implies that we have the restriction on $\zeta_{\beta}$ :

$$
\left|h \sum_{j=1}^{n-1} \lambda_{j}(z)\left(2 \beta_{j}+1\right)\right| \leq C h^{1 / m},
$$

for $0<1 / m<1$, giving

$$
\begin{aligned}
\#\left\{\zeta_{\beta}(z)\right\} & =\#\left\{\left|h \sum_{j=1}^{n-1} \lambda_{j}(z)\left(2 \beta_{j}+1\right)\right| \leq C h^{1 / m}\right\} \\
& \simeq \#\left\{|\beta| \leq h^{1 / m-1}\right\} \\
& \simeq h^{(1 / m-1)(n-1)}+o(1) .
\end{aligned}
$$

10.3. The proof of Theorem 7 . Observe the functions $v_{\beta}$ constructed above satisfy

$$
\mathrm{WF}_{h} v_{\beta}=(0,0) \in \mathbb{R}^{2 n-2} .
$$

Beginning with $v_{\beta}$ we want to construct $\tilde{v}_{\beta, k}$ and find values of $z, \beta$, and $k \in \mathbb{Z}$ so that

$$
(\text { id }-M(z)) \tilde{v}_{\beta}=\mathcal{O}\left(h^{\infty}\right) .
$$

Let

$$
\widetilde{M}(z)=V(z) M(z) V(z)^{-1}=e^{-i\left(Q(z, h)-T_{0} z\right) / h}
$$

with $V(z)$ and $Q(z, h)$ as in (10.10), and observe that $\widetilde{M}(z)=\widetilde{M}(z, 1)$ for

$$
\widetilde{M}(z, t)=\exp \left(-i t\left(Q(z, h)-T_{0} z\right) / h\right)
$$


satisfying

$$
\left\{\begin{array}{l}
h D_{t} \widetilde{M}(z, t)+Q(z, h) \widetilde{M}(z, t)=T_{0} z \widetilde{M}(z, t), \\
\widetilde{M}(z, 0)=\mathrm{id} .
\end{array}\right.
$$

The spectrum of $h D_{t}$ on $\mathbb{R} / \mathbb{Z}$ is $\{h 2 \pi k\}$ for $k \in \mathbb{Z}$, so we want to compare the solution space to (10.14) with the "ansatz" space

$$
v_{k, \beta}(t, x):=e^{i t 2 \pi k} v_{\beta}(x) .
$$

In other words, we want to choose values of $z, k, \beta$ so that

$$
\widetilde{M}(z, t) v_{\beta}(x)=e^{i t 2 \pi k} v_{\beta}(x)
$$

is just multiplication by a unimodular constant. More precisely, $v_{k, \beta}(1, x)=v_{\beta}(x)$, so we want to solve

$$
\left\{\begin{array}{l}
h D_{t} \widetilde{M}(z, t) v_{\beta, k}+\left(Q(z, h)-T_{0} z\right) \widetilde{M}(z, t) v_{\beta, k}=0, \\
\widetilde{M}(z, 0) v_{\beta, k}=v_{\beta, k} .
\end{array}\right.
$$

That is, we want to find $z$ satisfying

$$
T_{0} z-\zeta_{\beta}(z)=2 \pi k h,
$$

where $\zeta_{\beta}(z)$ is given by $(10.12)$.

We want to expand $Q(z, h)$ in a formal series in $z$. To do this, we note that derivatives of $Q(z, h)$ with respect to $z$ remain bounded operators, and since $z=$ $\mathcal{O}\left(h^{1 / m}\right)$, the formal Taylor expansion has some decay in powers of $h$. Hence applying the Borel lemma, we write

$$
Q(z, h)=\sum_{l=0}^{\infty} z^{l} Q_{l}(h, I)
$$

microlocally, with

$$
Q_{0}=\sum_{j=1}^{n-1} \lambda_{j}(0) I_{j}+Q_{0,1}(I)
$$

where

$$
Q_{0,1}=\mathcal{O}\left(I^{2}\right)
$$

and

$$
Q_{l}=\mathcal{O}(I)
$$

Hence we will seek

$$
z_{k, \beta}=\sum_{j=0}^{\infty} z_{k, \beta}^{(j)},
$$

with $z_{k, \beta}^{(j)}=\mathcal{O}\left(h^{(j+1) / m}\right)$. For $z_{k, \beta}^{(0)}$, we solve

$$
T_{0} z_{k, \beta}^{(0)}=h \sum_{j=1}^{n-1} \lambda_{j}(0)\left(2 \beta_{j}+1\right)+2 k \pi h,
$$


which is $\mathcal{O}\left(h^{1 / m}\right)$ if

$$
|k| \leq C h^{1 / m-1} .
$$

For $z_{k, \beta}^{(1)}$ we plug $z_{k, \beta}^{(0)}+z_{k, \beta}^{(1)}$ into (10.16), compare (10.17) to (10.12) and retain only the terms of order $h^{2 / m}$ to get the equation

$$
\begin{aligned}
T_{0} z_{k, \beta}^{(0)}+T_{0} z_{k, \beta}^{(1)} & =2 k \pi h+Q_{0}(h, h(2 \beta+1))+\sum_{l=1}^{\infty}\left(z_{k, \beta}^{(0)}+z_{k, \beta}^{(1)}\right)^{l} Q_{l}(h, h(2 \beta+\mathbb{1})) \\
& =T_{0} z_{k, \beta}^{(0)}+Q_{0,1}(h, h(2 \beta+1))+z_{k, \beta}^{(0)} Q_{1}(h, h(2 \beta+\mathbb{1}))+\mathcal{O}\left(h^{3 / m}\right),
\end{aligned}
$$

provided $z_{k, \beta}^{(1)}=\mathcal{O}\left(h^{2 / m}\right)$. Hence we choose

$$
T_{0} z_{k, \beta}^{(1)}=Q_{0,1}(h, h(2 \beta+1))+z_{k, \beta}^{(0)} Q_{l}(h, h(2 \beta+\mathbb{1})) .
$$

Continuing in this fashion, we select $z_{k, \beta}^{(j)}$ for $j \geq 2$ using the following equation:

$$
T_{0} \sum_{r=0}^{j} z_{k, \beta}^{(r)}=\sum_{r=0}^{j-1}\left(\sum_{l=0}^{j-r-1} z_{k, \beta}^{(l)}\right)^{r} Q_{r}(h, h(2 \beta+\mathbb{1})),
$$

modulo $\mathcal{O}\left(h^{(j+2) / m}\right)$; hence $z_{k, \beta}^{(j)}=\mathcal{O}\left(h^{(j+1) / m}\right)$.

Now there is no reason why (10.18) should converge in any sense, so we want to find a convergent series

$$
\tilde{z}_{k, \beta}=\sum_{j=0}^{\infty} \tilde{z}_{k, \beta}^{(j)}
$$

with $\tilde{z}_{k, \beta}^{(j)}=\mathcal{O}\left(h^{(j+1) / m}\right)$, satisfying

$$
\tilde{z}_{k, \beta}-\sum_{0}^{m N} z_{k, \beta}^{(j)}=\mathcal{O}\left(h^{N}\right)
$$

for every $N>0$. For this, we follow the proof of Borel's Lemma from DiSj], Chapter $2]$. Choose $\chi \in \mathcal{C}_{c}^{\infty}([-1,2])$ satisfying $\chi \equiv 1$ on $[0,1]$. Set

$$
\tilde{z}_{k, \beta}=\sum_{j=0}^{\infty} \chi\left(\lambda_{j} h\right) z_{k, \beta}^{(j)},
$$

where $\lambda_{j} \rightarrow \infty$, and $\lambda_{j}<\lambda_{j+1}$ has yet to be selected. Observe for each $h>0$, that this is a finite sum, hence converges. We calculate:

$$
\begin{aligned}
\tilde{z}_{k, \beta}-\sum_{j=0}^{m N+m} z_{k, \beta}^{(j)} & =\sum_{m N+m+1}^{\infty} \chi\left(\lambda_{j} h\right) z_{k, \beta}^{(j)}+\sum_{0}^{m N+m} z_{k, \beta}^{(j)}\left(\chi\left(\lambda_{j} h\right)-1\right) \\
& =: A+B .
\end{aligned}
$$


But since $x \chi(x)$ is uniformly bounded, we have

$$
\begin{aligned}
|A| & \leq \sum_{m N+m+1}^{\infty} C_{j} h^{(j+1) / m} \frac{\lambda_{j} h}{\lambda_{j} h} \chi\left(\lambda_{j} h\right) \\
& \leq \sum_{m N+m+1}^{\infty} C_{j}^{\prime} h^{(j-m+1) / m} \lambda_{j}^{-1} \\
& \leq h^{N} \sum_{m N+1}^{\infty} 2^{-j}
\end{aligned}
$$

if $\lambda_{j}$ is sufficiently large.

To estimate $B$, we observe for $0<\lambda_{m N+m} h \leq 1$, that $B=0$ since $\chi \equiv 1$ on $[0,1]$. If $\lambda_{m N+m}<h$, we calculate

$$
\begin{aligned}
|B| & \leq \sum_{0}^{m N+m} C_{j} h^{(j+1) / m}\left(\chi\left(\lambda_{j} h\right)-1\right) \\
& \leq C_{N} h^{1 / m} \lambda_{m N+m}^{N} h^{N},
\end{aligned}
$$

which is 10.20 ).

Now for fixed $(\beta, k)$ and $N>0$, we have the crude estimate

$$
\tilde{z}_{\beta, k}^{l}-\left(\sum_{j=0}^{m N+m}\left(z_{k, \beta}^{(j)}\right)\right)^{l}=\left(\tilde{z}_{\beta, k}-\sum_{j=0}^{m N+m}\left(z_{k, \beta}^{(j)}\right)\right)(l \mathcal{O}(1)),
$$

which from the definitions of $z_{k, \beta}, \tilde{z}_{k, \beta}$, and $Q_{l}(h, I)$ gives:

$$
\begin{aligned}
& h D_{t} \widetilde{M}\left(\tilde{z}_{k, \beta}, t\right) v_{\beta}+\left(Q\left(\tilde{z}_{k, \beta}, t\right)-T_{0} \tilde{z}_{k, \beta}\right) \widetilde{M}\left(\tilde{z}_{k, \beta}, t\right) v_{\beta} \\
& =\quad h D_{t} \widetilde{M}\left(\tilde{z}_{k, \beta}, t\right) v_{\beta} \\
& \quad+\left(\sum_{l=0}^{m N+m}\left(\sum_{j=0}^{m N}\left(z_{k, \beta}^{(j)}\right)\right) Q_{l}(h, h(2 \beta+\mathbb{1}))-T_{0} \sum_{j=0}^{m N+m}\left(z_{k, \beta}^{(j)}\right)\right) \widetilde{M}\left(\tilde{z}_{k, \beta}, t\right) v_{\beta} \\
& \quad+\mathcal{O}\left(h^{N}\right)\left\|v_{\beta}\right\|_{L^{2}\left(\mathbb{R}^{n-1}\right)} \\
& \quad(2 k \pi h) \widetilde{M}\left(\tilde{z}_{k, \beta}, t\right) v_{\beta}+\mathcal{O}\left(h^{N}\right)\left\|v_{\beta}\right\|_{L^{2}\left(\mathbb{R}^{n-1}\right)} .
\end{aligned}
$$

Hence

$$
\widetilde{M}\left(\tilde{z}_{k, \beta}, t\right) v_{\beta}=e^{i t 2 \pi k} v_{\beta}+t \mathcal{O}\left(h^{N-1}\right)\left\|v_{\beta}\right\|_{L^{2}\left(\mathbb{R}^{n-1}\right)},
$$

so

$$
\left(\mathrm{id}-\widetilde{M}\left(\tilde{z}_{k, \beta}\right)\right) v_{\beta}=\mathcal{O}\left(h^{N-1}\right)\left\|v_{\beta}\right\|_{L^{2}\left(\mathbb{R}^{n-1}\right)}
$$

for any $N$, or

$$
\left(\text { id }-\widetilde{M}\left(\tilde{z}_{k, \beta}\right)\right) v_{\beta}=\mathcal{O}\left(h^{\infty}\right)\left\|v_{\beta}\right\|_{L^{2}\left(\mathbb{R}^{n-1}\right)} .
$$

Now the definition of $\widetilde{M}$ implies

$$
M\left(\tilde{z}_{k, \beta}\right) V\left(\tilde{z}_{k, \beta}\right)^{-1} v_{\beta}=V\left(\tilde{z}_{k, \beta}\right)^{-1} v_{\beta}+\mathcal{O}\left(h^{\infty}\right)\left\|V\left(\tilde{z}_{k, \beta}\right)^{-1} v_{\beta}\right\|_{L^{2}\left(\mathbb{R}^{n-1}\right)},
$$


so

$$
u_{\tilde{z}_{k, \beta}}:=E_{+} V\left(\tilde{z}_{k, \beta}\right)^{-1} v_{\beta},
$$

with $E_{+}$defined in (5.4) satisfying (10.3).

Finally, the quantization conditions (10.8)-(10.9) and the estimates (10.13) and (10.19) give

$$
\#\left\{z:(Q(z, h)-z) v=\mathcal{O}\left(h^{\infty}\right)\right\} \geq C^{-1} h^{-n(1-1 / m)},
$$

which is 10.4.

\section{Appendix A. SEmi-Hyperbolic geodesics in 3 Dimensions}

In this appendix, we modify the example of Colin de Verdière-Parisse CVP to extend to three dimensions and have a semi-hyperbolic geodesic.

Consider the Riemannian manifold

$$
M=\mathbb{R}_{x} / \mathbb{Z} \times \mathbb{R}_{y} \times \mathbb{R}_{z}
$$

equipped with the metric

$$
d s^{2}=\cosh ^{2} y\left(2 z^{4}-z^{2}+1\right)^{2} d z^{2}+d y^{2}+d z^{2} .
$$

Thus the matrix for the metric is given by

$$
g_{i j}=\left\{\begin{array}{l}
\cosh ^{2} y\left(2 z^{4}-z^{2}+1\right)^{2}, i=j=1, \\
1, i=j=2,3 \\
0, i \neq j
\end{array}\right.
$$

and we calculate the Christoffel symbols:

$$
\begin{aligned}
& \Gamma_{2,1}^{1}=\Gamma_{1,2}^{1}=\tanh y, \\
& \Gamma_{3,1}^{1}=\Gamma_{1,3}^{1}=\left(8 z^{3}-2 z\right)\left(2 z^{4}-z^{2}+1\right)^{-1}, \\
& \Gamma_{1,1}^{2}=-\sinh y \cosh y\left(2 z^{4}-z^{2}+1\right)^{2}, \\
& \Gamma_{1,1}^{3}=-\left(8 z^{3}-2 z\right)\left(2 z^{4}-z^{2}+1\right) \cosh ^{2} y,
\end{aligned}
$$

with all other Christoffel symbols equal to zero. The geodesic equations are

$$
\begin{aligned}
\ddot{x} & =-2(\tanh y) \dot{y} \dot{x}-2\left(\left(8 z^{3}-2 z\right)\left(2 z^{4}-z^{2}+1\right)^{-1}\right) \dot{z} \dot{x}, \\
\ddot{y} & =\sinh y \cosh y\left(2 z^{4}-z^{2}+1\right)^{2} \dot{x}^{2}, \\
\ddot{z} & =\left(8 z^{3}-2 z\right)\left(2 z^{4}-z^{2}+1\right) \cosh ^{2} y \dot{x}^{2} .
\end{aligned}
$$

Setting $v_{x}=\dot{x}, v_{y}=\dot{y}$, and $v_{z}=\dot{z}$, we get the first-order system

$$
\begin{aligned}
\dot{x} & =v_{x}, \\
\dot{v}_{x} & =-2(\tanh y) v_{y} v_{x}-2\left(\left(8 z^{3}-2 z\right)\left(2 z^{4}-z^{2}+1\right)^{-1}\right) v_{z} v_{x}, \\
\dot{y} & =v_{y}, \\
\dot{v}_{y} & =\sinh y \cosh y\left(2 z^{4}-z^{2}+1\right)^{2} v_{x}^{2}, \\
\dot{z} & =v_{z}, \\
\dot{v}_{z} & =\left(8 z^{3}-2 z\right)\left(2 z^{4}-z^{2}+1\right) \cosh ^{2} y v_{x}^{2} .
\end{aligned}
$$

There are trivially three periodic geodesics, given by the solutions

$$
\begin{aligned}
& x(t)=v_{x}(0) t+x(0), \\
& y(t)=0, \\
& z(t)=0, \pm 1 / 2 .
\end{aligned}
$$




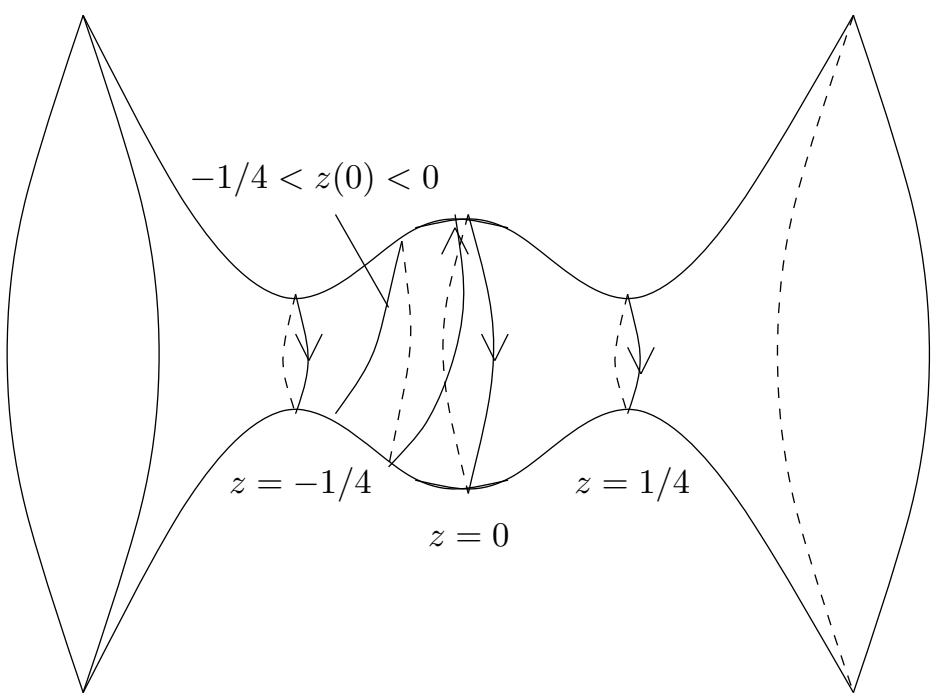

FiguRE 8. The $x-z$ hypersurface in $M$ with some representative orbits.

Next we examine the Laplace-Beltrami operator on $M$. We compute

$$
\begin{aligned}
\Delta= & |g|^{-1 / 2} \partial_{i}|g|^{1 / 2} g^{i j} \partial_{j} \\
= & \cosh ^{-2} y\left(2 z^{4}-z^{2}+1\right)^{-2} \partial_{x}^{2}+\partial_{y}^{2}+\partial_{z}^{2} \\
& \quad+\tanh y \partial_{y}+\left(8 z^{3}-2 z\right)\left(z^{4}-z^{2}+1\right)^{-1} \partial_{z} .
\end{aligned}
$$

The isometry $T: L^{2}\left(M, d \operatorname{Vol}_{g}\right) \rightarrow L^{2}(M, d x d y d z)$ given by

$$
T u(x, y, z)=\cosh ^{1 / 2} y\left(2 z^{4}-z^{2}+1\right)^{1 / 2} u(x, y, z)
$$

conjugates $\Delta$ into a selfadjoint operator $\widetilde{\Delta}$. A computation yields

$$
\begin{aligned}
\widetilde{\Delta}= & T \Delta T^{-1} \\
= & \cosh ^{-2} y\left(2 z^{4}-z^{2}+1\right)^{-2} \partial_{x}^{2}+\partial_{y}^{2}+\partial_{z}^{2}-\frac{1}{4}\left(1+\operatorname{sech}^{2} y\right) \\
& \quad+\frac{1}{4}\left(8 z^{3}-2 z\right)^{2}\left(2 z^{4}-z^{2}+1\right)^{-2}-\frac{1}{2}\left(24 z^{2}-2\right)\left(2 z^{4}-z^{2}+1\right)^{-1} .
\end{aligned}
$$

In keeping with the theme of this work, we want to examine the asymptotic behaviour of eigenfunctions for this operator. In order to separate variables, let

$$
\varphi_{k, \lambda}(x, y, z)=e^{i k x} \psi_{k, \lambda}(y, z)
$$

and compute:

$$
\begin{aligned}
-\widetilde{\Delta} \varphi_{k, \lambda}= & \left(-\Delta_{y, z}+k^{2} \cosh ^{-2} y\left(2 z^{4}-z^{2}+1\right)^{-2}+\frac{1}{4}\left(1+\operatorname{sech}^{2} y\right)\right. \\
& \left.-\frac{1}{4}\left(8 z^{3}-2 z\right)^{2}\left(2 z^{4}-z^{2}+1\right)^{-2}+\frac{1}{2}\left(24 z^{2}-2\right)\left(2 z^{4}-z^{2}+1\right)^{-1}\right) \varphi_{k, \lambda} .
\end{aligned}
$$


Rearranging, we have the following equation for $\psi_{k, \lambda}$ :

$$
\begin{gathered}
\left(-\Delta_{y, z}+k^{2}\left(\cosh ^{-2} y\left(2 z^{4}-z^{2}+1\right)^{-2}-1\right)\right. \\
+\frac{1}{4}\left(1+\operatorname{sech}^{2} y\right)-\frac{1}{4}\left(8 z^{3}-2 z\right)^{2}\left(2 z^{4}-z^{2}+1\right)^{-2} \\
\left.+\frac{1}{2}\left(24 z^{2}-2\right)\left(2 z^{4}-z^{2}+1\right)^{-1}\right) \psi_{k, \lambda} \\
=\left(\lambda-k^{2}\right) \psi_{k, \lambda} .
\end{gathered}
$$

We divide by $k^{2}$ and use $h=1 / k$ as the semiclassical parameter, giving

$$
\begin{aligned}
P(h) \psi_{h} & =\left(-h^{2} \Delta_{y z}+V(y, z)\right) \psi_{h} \\
& =\left(h^{2} \lambda-1\right) \psi_{h},
\end{aligned}
$$

with

$$
\begin{aligned}
V(y, z)= & \cosh ^{-2} y\left(2 z^{4}-z^{2}+1\right)^{-2}-1 \\
& h^{2} \frac{1}{4}\left(1+\operatorname{sech}^{2} y\right)-h^{2} \frac{1}{4}\left(8 z^{3}-2 z\right)^{2}\left(2 z^{4}-z^{2}+1\right)^{-2} \\
& +h^{2} \frac{1}{2}\left(24 z^{2}-2\right)\left(2 z^{4}-z^{2}+1\right)^{-1} .
\end{aligned}
$$

The semiclassical principal symbol is

$$
\begin{aligned}
\sigma_{h}(P) & =\eta^{2}+\zeta^{2}+\cosh ^{-2} y\left(2 z^{4}-z^{2}+1\right)^{-2}-1 \\
& =: \eta^{2}+\zeta^{2}+\widetilde{V} .
\end{aligned}
$$

Observe that $\widetilde{V}$ has nondegenerate critical points at $y=0, z=0, \pm 1 / 4$. See Figure 8. The signatures of $\partial^{2} \widetilde{V}$ are $(-,+),(-,-)$, and $(-,-)$, respectively. Thus the quadratic part of the normal forms for $\sigma_{h}(P)$ takes the form

$$
\begin{aligned}
& \lambda_{1} y \eta+\frac{\lambda_{2}}{2}\left(z^{2}+\zeta^{2}\right), \text { near } y=0, z=0, \text { and } \\
& \lambda_{1} y \eta+\lambda_{2} z \zeta, \text { near } y=0, z= \pm 1 / 4 .
\end{aligned}
$$

\section{ACKNOWLEDGEMENTS}

The author would like to thank Maciej Zworski for much help and support during the writing of this work, as well as Nicolas Burq for suggesting the study of the monodromy operator as a means of tackling the boundary problem. He would also like to thank Herbert Koch for suggesting the generalization to semi-hyperbolic orbits, and Michael Hitrik for much help in working out the model case for Theorem 7. The majority of this work was conducted while the author was a graduate student in the Mathematics Department at UC-Berkeley and he is very grateful for the support received while there. Finally, the author would like to thank the anonymous referees and readers whose many suggestions have greatly helped to improve the exposition of this work.

\section{REFERENCES}

[AbMa] Abraham, R., Marsden, J., Kelley, A. and Kolmogorov, A.N. Foundations of Mechanics. W. A. Benjamin, Inc., New York, 1967. MR0220467 (36:3527)

[AuMa] Aurich, R. and Marklof, J. Trace Formulae for 3-dimensional Hyperbolic Lattices and Application to a Strongly Chaotic Tetrahedral Billiard. Phys. D. 92, No. 1-2 (1996), 101-129. MR 1384681 (97f:81035) 
[BoCh] Bony, J.-M. and Chemin, J.-Y. Espaces fonctionnels associés au calcul de WeylHörmander. Bull. Soc. Math. France 122 (1994), 77-118. MR.1259109 (95a:35152)

[Bur] Burq, N. Smoothing Effect for Schrödinger Boundary Value Problems. Duke Math. Journal. 123, No. 2 (2004), 403-427. MR2066943(2006e:35026)

[BuCh] Burq, N. and Christianson, H. Imperfect geometric control and overdamping for the damped wave equation. in preparation.

[BuZw] Burq, N. and Zworski, M. Geometric Control in the Presence of a Black Box. J. Amer. Math. Soc. 17 (2004), 443-471. MR2051618(2005d:47085)

[CaPo] Cardoso, F. and Popov, G. Quasimodes with exponentially small errors associated with elliptic periodic rays. Asymptot. Anal. 30, No. 3-4 (2002), 217-247. MR 1932033 (2003h:35181)

[Chr1] Christianson, H. Semiclassical Non-concentration near Hyperbolic Orbits. J. Funct. Anal. 246 (2007), no. 2, 145-195. MR2321040(2008k:58058)

[Chr1a] Christianson, H. Corrigendum to "Semiclassical non-concentration near hyperbolic orbits" [J. Funct. Anal. 246 (2) (2007) 145-195]. J. Funct. Anal. 258 (2010) No. 3, 10601065. MR2558187(2010i:58024)

[Chr2] Christianson, H. Dispersive Estimates for Manifolds with one Trapped Orbit. Commun. PDE, 33 (2008) No. 7, 1147-1174. MR2450154(2009i:35297)

[Chr3] Christianson, H. Applications of Cutoff Resolvent Estimates to the Wave Equation. Math. Res. Lett., 16 (2009), No. 4, 577-590. MR2525026

[ChWu] Christianson, H. and Wunsch, J. Local smoothing for the Schrödinger equation with a prescribed loss. in preparation.

[CdV] Colin de Verdière, Y. Quasi-modes sur les variétés Riemanniennes. Invent. Math., 43, No. 1 (1977), 15-52. MR0501196 (58:18615)

[CVP] Colin de Verdière, Y. and Parisse, B. Equilibre Instable en Régime Semi-classique: I Concentration Microlocale. Commun. PDE. 19 (1994), 1535-1563; Equilibre Instable en Régime Semi-classique: II - conditions de Bohr-Sommerfeld. Ann. Inst. Henri Poincaré (Phyique théorique). 61 (1994), 347-367. MR1294470(96b:58112)

[DiSj] Dimassi, M. and Sjöstrand, J. Spectral Asymptotics in the Semi-classical Limit. Cambridge University Press, Cambridge, 1999. MR.1735654 (2001b:35237)

[EvZw] Evans, L.C. and Zworski, M. Lectures on Semiclassical Analysis. http://math. berkeley.edu/ evans/semiclassical.pdf.

[HaKa] Hasselblatt, B. and Katok, A. Introduction to the Modern Theory of Dynamical Systems. University of Cambridge Press, Cambridge, 1995. MR.1326374 (96c:58055)

[HeSj] Helffer, B. and Sjöstrand, J. Semiclassical analysis for Harper's equation. III. Cantor structure of the spectrum. Mém. Soc. Math. France. 39 (1989), 1-124. MR1041490 (92m:35011)

[HoZe] Hofer, H. and Zehnder, E. Symplectic Invariants and Hamiltonian Dynamics. Birkhäuser Verlag, Basel, 1994. MR1306732 (96g:58001)

[Hor1] Hörmander, L. The Analysis of Linear Partial Differential Operators I. Springer-Verlag, Berlin, 1983. MR717035 (85g:35002a)

[Hor3] Hörmander, L. The Analysis of Linear Partial Differential Operators III. SpringerVerlag, Berlin, 1985. MR781536 (87d:35002a)

[IaSj] Iantchenko, A. and Sjöstrand, J. Birkhoff Normal Forms for Fourier Integral Operators II. American Journal of Mathematics. 124 (2002), 817-850. MR1914459 (2003f:58054)

[ISZ] Iantchenko, A., Sjöstrand, J., and Zworski, M. Birkhoff Normal Forms in Semiclassical Inverse Problems. Math. Res. Lett. 9 (2002), 337-362. MR.1909649 (2003f:35284)

[MeSj1] Melrose, R. B.; Sjöstrand, J. Singularities of boundary value problems. I. Comm. Pure Appl. Math. 31 (1978), No. 5, 593-617. MR0492794 (58:11859)

[MeSj2] Melrose, R. B.; Sjöstrand, J. Singularities of boundary value problems. II. Comm. Pure Appl. Math. 35 (1982), No. 2, 129-168. MR644020 (83h:35120)

[NoZw1] Nonnenmacher, S. and Zworski, M. Quantum decay rates in chaotic scattering. Acta Math. 203 (2009), No. 2, 149-233. MR2570070

[NoZw2] Nonnenmacher, S. and Zworski, M. Semiclassical Resolvent Estimates in Chaotic Scattering. Appl. Math. Res. Express. AMRX (2009) 2009:74-86, doi:10.1093/amrx/abp003.

[Ral] Ralson, J. Trapped rays in spherically symmetric media and poles of the scattering matrix. Comm. Pure Appl. Math. 24 (1971), 571-582. MR0457962(56:16166) 
[Sjö] Sjöstrand, J. Geometric Bounds on the Density of Resonances for Semiclassical Problems. Duke Mathematical Journal. 60, No. 1 (1990), 1-57. MR.1047116 (91e:35166)

[SjZw1] Sjöstrand, J. and Zworski, M. Quantum Monodromy and Semi-classical Trace Formulæ. Journal de Mathématiques Pures et Appliquées. 81 (2002), 1-33. MR.1994881 (2004g:58035)

[SjZw2] Sjöstrand, J. and Zworski, M. Quantum Monodromy Revisited. http://www.math. berkeley.edu/'zworski.

[SjZw3] Sjöstrand, J. and Zworski, M. Fractal Upper Bounds on the Density of Semiclassical Resonances. http://xxx.lanl.gov/pdf/math.SP/0506307.

[TaZw] Tang, S.H. and Zworski, M. From Quasimodes to Resonances. Math. Res. Lett. 5 (1998), 261-272. MR 1637824 (99i:47088)

[Tay1] Taylor, M. Partial differential equations. I. Basic theory. Applied Mathematical Sciences, 115. Springer-Verlag, New York, 1996. MR.1395148(98b:35002b)

Department of Mathematics, Massachusetts Institute of Technology, 77 MassachuSetts Avenue, Cambridge, Massachusetts 02139

E-mail address: hans@math.mit.edu

Current address: Department of Mathematics, University of North Carolina-Chapel Hill, CB\#3250 Phillips Hall, Chapel Hill, North Carolina 27599

E-mail address: hans@math.unc.edu 